Florida International University

FIU Digital Commons

2-28-2019

\title{
Cognitive Factors and Parasympathetic Regulation as Interacting Mechanisms of Attention-Deficit/Hyperactivity Disorder
}

Anthony R. Ward

award019@fiu.edu

Follow this and additional works at: https://digitalcommons.fiu.edu/etd

Part of the Biological Psychology Commons, Child Psychology Commons, Clinical Psychology Commons, and the Cognitive Psychology Commons

\section{Recommended Citation}

Ward, Anthony R., "Cognitive Factors and Parasympathetic Regulation as Interacting Mechanisms of Attention-Deficit/Hyperactivity Disorder" (2019). FIU Electronic Theses and Dissertations. 4057.

https://digitalcommons.fiu.edu/etd/4057

This work is brought to you for free and open access by the University Graduate School at FIU Digital Commons. It has been accepted for inclusion in FIU Electronic Theses and Dissertations by an authorized administrator of FIU Digital Commons. For more information, please contact dcc@fiu.edu. 


\section{FLORIDA INTERNATIONAL UNIVERSITY}

Miami, Florida

\section{COGNITIVE FACTORS AND PARASYMPATHETIC REGULATION AS \\ INTERACTING MECHANISMS OF ATTENTION-DEFICIT/HYPERACTIVITY DISORDER}

A dissertation submitted in partial fulfillment of

the requirements for the degree of

DOCTOR OF PHILOSOPHY

in

PSYCHOLOGY

by

Anthony Robert Ward 
To: Dean Michael R. Heithaus

College of Arts, Sciences and Education

This dissertation, written by Anthony Robert Ward, and entitled Cognitive Factors and Parasympathetic Regulation as Interacting Mechanisms of Attention-Deficit/Hyperactivity Disorder, having been approved in respect to style and intellectual content, is referred to you for judgment.

We have read this dissertation and recommend that it be approved.

$\begin{array}{r}\hline \text { Raul Gonzalez } \\ \hline \text { Joseph S. Raiker } \\ \hline \text { Barbara Thomlison } \\ \hline \text { Erica D. Musser, Major Professor }\end{array}$

Date of Defense: February 28, 2019

The dissertation of Anthony Robert Ward is approved.

Dean Michael R. Heithaus College of Arts, Sciences and Education

Andrés G. Gil

Vice President for Research and Economic Development and Dean of the University Graduate School

Florida International University, 2019 


\section{DEDICATION}

I dedicate this dissertation to my wife and children, whose love motivated me to persevere through all difficulties and self-doubt.

To my wife especially: Hikari, this was only possible because of your patience, support, and incredible love. SMILY! 


\section{ACKNOWLEDGMENTS}

I wish to thank Dr. Erica Musser, my major professor, for her mentorship and substantial commitment of time and resources toward this project.

I would also like to thank the other faculty members within the Clinical Science in Child and Adolescent Psychology program at FIU, whose hard work has resulted in an impressive and productive program in a very short time. Furthermore, their commitment to the development and success of doctoral students was evident. I also thank the staff of the ABC-ERICA Lab, under the direction of Dr. Musser, who helped substantially with data collection and processing. In particular, to Jessica Martinez and Yulie Lugo, and my fellow doctoral student colleagues Rachel Tenenbaum and Stephanie Morris - thank you for your help and encouragement over these past years. Thank you also to student colleagues (like Tommy Chou, and others!) who became life-long friends.

I also acknowledge the children and families who participated in the various research programs and clinical experiences that have shaped my training over the past several years.

Finally, acknowledgement and sincerest thanks go out to my family: thank you Mom, Dad, AJ, Chris, Kelcey, Mallory, Grandpa Ed, Kathy, and Nanny and Pop for your love, support, and encouragement. 


\section{ABSTRACT OF THE DISSERTATION \\ COGNITIVE FACTORS AND PARASYMPATHETIC REGULATION AS \\ INTERACTING MECHANISMS OF ATTENTION-DEFICIT/HYPERACTIVITY}

\section{DISORDER}

by

Anthony Robert Ward

Florida International University, 2019

Miami, Florida

Professor Erica D. Musser, Major Professor

Attention-deficit/hyperactivity disorder (ADHD) is among the most commonly diagnosed mental health disorders in childhood, and yet, the causal mechanisms of the disorder remain unclear. Deficits in attention regulation, inhibition, and working memory are frequently proposed as core mechanisms of ADHD, but these deficits are highly heterogeneous at the individual level, which hampers advances in understanding the etiology of the disorder. Recent research has shown that parasympathetic nervous system (PNS) regulation is linked to cognitive function and emotion regulation; atypical PNS regulation is associated with problems in these domains as well as higher risk for psychopathology overall. This dissertation examined aspects of attention, response inhibition, and working memory, as well as PNS regulation and reactivity, in a sample of children both with and without ADHD; between-groups comparisons were conducted using ANCOVA, as well as examining associations with a continuous measure of ADHD symptom severity using multiple regression. Furthermore, the possible moderating effect 
of PNS reactivity on the association between each cognitive domain and ADHD was evaluated.

Children with ADHD exhibited significantly poorer performance on tests of attention, response inhibition, and attentional regulation compared to typically developing youth. Parasympathetic regulation at rest was also significantly reduced among ADHD youth compared to typically developing peers. In addition, there was a consistent moderating effect of PNS reactivity on the continuous associations between each cognitive domain and inattention symptom severity. The possible relevance of these findings is discussed with respect to multiple pathway and additive models of ADHD development, as well as cognitive-energetic etiological models which hypothesize deficits in broad regulatory capacities which cascade into executive functioning difficulties. Future directions are also noted and include the need to examine similar interactions within a longitudinal design, and the need to describe the role of PNS regulation in the development of ADHD in greater detail. 


\section{TABLE OF CONTENTS}

CHAPTER

PAGE

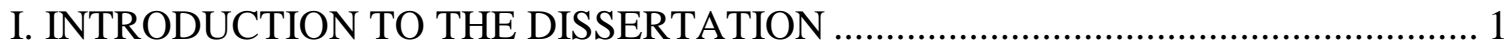

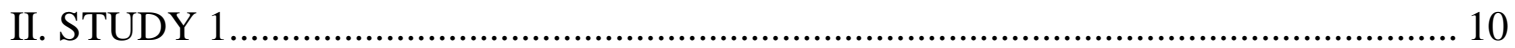

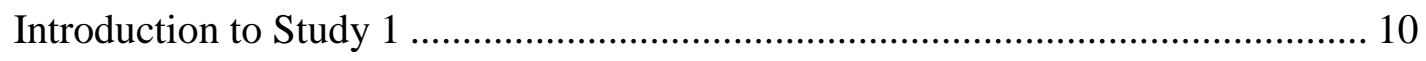

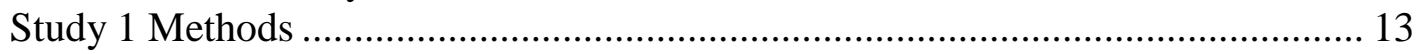

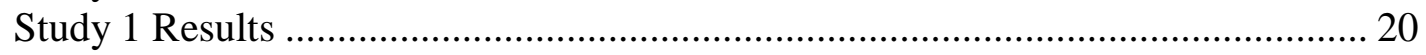

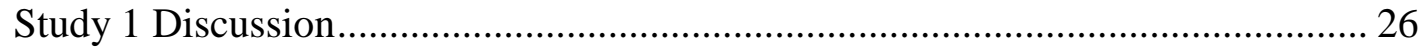

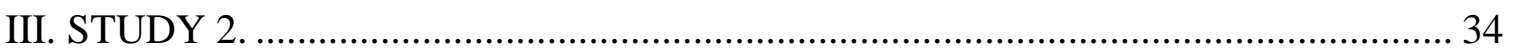

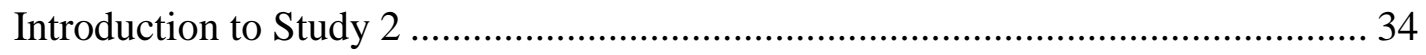

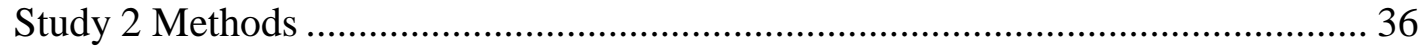

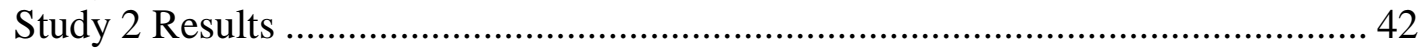

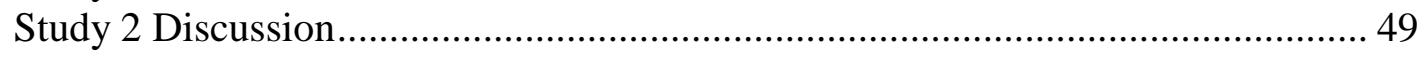

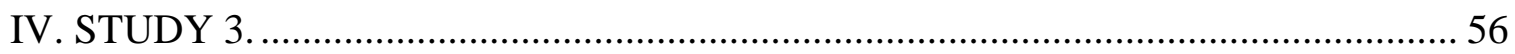

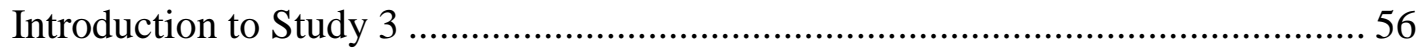

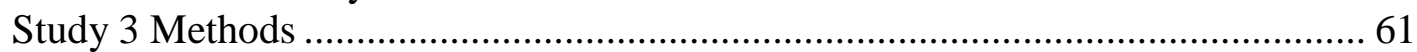

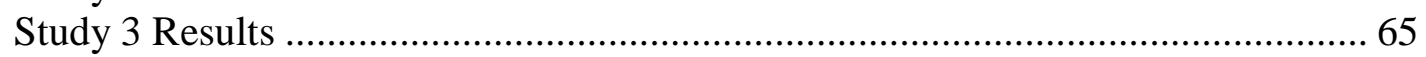

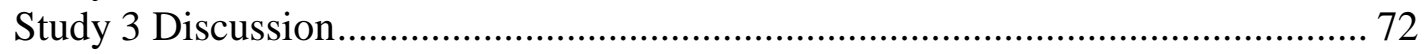

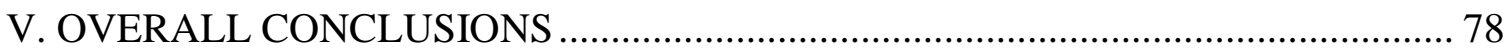

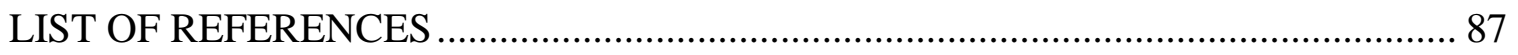

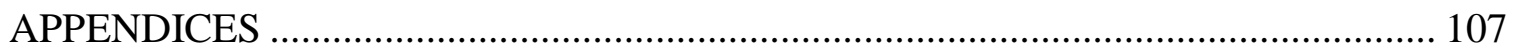

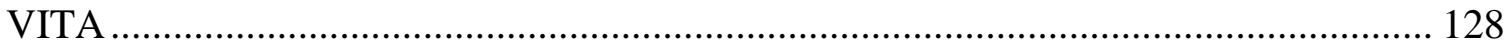




\section{LIST OF TABLES}

TABLE

PAGE

1. Demographic and Diagnostic Characteristics for ADHD and Control Groups Study 1 108

2. Inter-Correlations among Clinical, WM, and RSA Indices

3a. Estimated Marginal Means and ANCOVA Results for WM and RSA Variables.....110

3b. Raw Means for WM and RSA Variables with Between-Groups Comparisons ........110

4a. Results of Regression Models Predicting Mean Rating of Overall ADHD Symptoms

4b. Results of Regression Models Predicting Mean Rating of Inattentive Symptoms ....111

4c. Results of Regression Models Predicting Mean Rating of Hyperactive/Impulsive Symptoms

5. Conditional Effects - WM Predicting Mean ADHD Rating by level of RSAChange

6. Demographic and Diagnostic Characteristics for ADHD and Control Groups for Study 2

7a. Correlations Among Clinical, Go/No-Go, and RSA Variables - Entire Sample.

7b. Correlations Among Clinical, Go/No-Go, and RSA Variables -ADHD Sample Only

8a. Estimated Marginal Means and ANCOVA Results for Go/No-Go and RSA Variables

8b. Raw Means for Go/No-Go and RSA with Between-Groups Comparison

9a. Results of Regression Models Predicting Mean Rating for ADHD Symptoms Study 2

9b. Results of Regression Models Predicting Mean Rating for Inattentive Symptoms -

Study 2

9c. Results of Regression Models Predicting Mean Rating for Hyperactive/Impulsive Symptoms - Study 2 
10. Conditional Effects - Total GNG Errors Predicting Mean ADHD Rating, by Level of RSA-Change.

11a. Correlations Among Clinical, RTV, and RSA Variables - Entire Sample.

11b. Correlations Among Clinical, RTV, and RSA Variables within ADHD Group .....121

12a. Estimated Marginal Means and ANCOVA Results for RTV Variables

12b. Raw Means for RT and RTV Variables with Between-Groups Comparison

13a. Results of Regression Models Predicting Mean Rating for ADHD Symptoms Study 3

13b. Results of Regression Models Predicting Mean Rating for Inattentive Symptoms - Study 3

13c. Results of Regression Models Predicting Mean Rating for Hyperactive/ Impulsive Symptoms - Study 3

14a. Conditional Effects - SDRT predicting ADHD Symptom Severity, by level of RSA-Change

14b. Conditional Effects - Tau predicting ADHD Symptom Severity, by level of RSA-Change 


\section{LIST OF FIGURES}

FIGURE

PAGE

1. Interaction of RSA Change and WM Predicting Overall ADHD Raw Score............ 113

2. Mean RSA Across Each Period, Per Group - Study 1............................................ 113

3. Interaction of RSA Change and GNG Errors Predicting Mean Inattentive Symptom

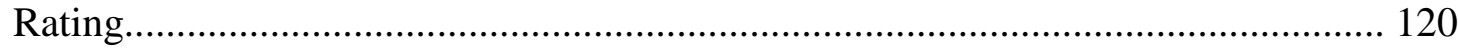

4. ex-Gaussian Probability Function.................................................................................. 125

5. Interaction of SDRT and RSA-Change Predicting Mean Inattentive Symptom Rating. 126

6. Interaction of Tau and RSA-Change Predicting Mean Inattentive Symptom Rating. 


\section{LIST OF ABBREVIATIONS AND ACRONYMS}

American Psychiatric Association

APA

Attention-Deficit/Hyperactivity Disorder

ADHD

Conduct Disorder

$\mathrm{CD}$

Diagnostic and Statistical Manual of Mental Disorders, $4{ }^{\text {th }}$ Edition

DSM-IV

Diagnostic and Statistical Manual of Mental Disorders, $5^{\text {th }}$ Edition

DSM -5

Diagnostic Interview Schedule - Child Version

DISC-IV

Disruptive Behavior Disorders Rating Scale

DBD-RS

Electrocardiogram

ECG

Estimated Marginal Mean

EMM

Go/No-Go Task

GNG

Impedance Cardiogram

ICG

Mean Reaction Time

MRT

Oppositional Defiant Disorder

ODD

Parasympathetic Nervous System

PNS

Respiratory Sinus Arrhythmia

RSA

Reaction Time

RT

Reaction Time Variability

RTV

Standard Deviation of Reaction Time

SDRT

Typically Developing

TD

Working Memory

WM 


\section{CHAPTER I. INTRODUCTION TO THE DISSERTATION}

Attention-deficit/hyperactivity disorder (ADHD) is a childhood-onset neurodevelopmental disorder characterized by inattention, hyperactivity, and/or impulsivity, which lead to substantial impairment across multiple settings (American Psychiatric Association, 2013). The disorder is prevalent with epidemiological studies estimating between 7 and $9 \%$ of school-age children meet criteria for the diagnosis (Pastor \& Reuben, 2008; Thomas et al., 2015). Further, ADHD persists throughout adolescence and adulthood for many individuals (Biederman, Petty, Clarke, Lomedico, \& Faraone, 2010; Langberg et al., 2008; Kessler et al., 2006). The estimated economic impact of ADHD with regard to treatment, education, family productivity, and criminal justice costs is estimated at $\$ 42.5$ billion annually (Pelham, Foster, \& Robb, 2007). Furthermore, ADHD in childhood is a risk factor for a host of negative health, psychosocial, and economic outcomes across development (Barkley, Anastopoulus, Guevremont \& Fletcher, 1991; Barkley, Fischer, Smallish, \& Fletcher, 2004; Molina \& Pelham, 2003). Thus, commensurate with the prevalence, high costs, and public health impact associated with ADHD, research focusing on etiology and potential prevention and intervention programs has progressed for decades and remains a high priority for the field.

Diagnosis of ADHD relies on reports of observable behaviors in at least one of two domains: inattentive symptoms and/or hyperactive/impulsive symptoms (American Psychiatric Association, 2013). However, these symptom domains, as well as the three DSM-5 presentations of ADHD, do not appear to correspond with substantial differences 
in impairment, neurocognitive features, or response to treatment. Prior to publication of the DSM-5, a meta-analytic review authored by the ADHD subcommittee of the Disruptive Behavior Disorders Work Group described the DSM-IV subtypes as "convenient clinical shorthand" which lack corresponding etiological correlates and longterm prognostic specificity (Willcutt et al., 2012, p.2).

\section{Cognitive Heterogeneity in ADHD}

These broad issues are emblematic of research over recent decades, which has shown that children with ADHD likely constitute a highly heterogeneous group with regard to underlying cognitive and developmental mechanisms. For example, numerous theories have proposed weaknesses in domains of executive functioning - the top-down cognitive processes involved in regulating goal-directed cognition and behavior - as core to the etiology of ADHD (Barkley, 1997; Castellanos \& Tannock, 2002; Rapport, Chung, Shore, \& Isaacs, 2001). However, while children with ADHD exhibit substantial impairment in these domains at the group level, up to half of individual children with ADHD perform within the normative range on these tasks (see Nigg et al., 2005). Additionally, there is substantial within-person variability across task performance among individuals with ADHD (Karalunas, Geurts, Konrad, Bender, Nigg, 2014; Kofler et al., 2013). These findings lend support to theories of ADHD as being rooted in multiple etiological pathways (Nigg, Goldsmith \& Sachek, 2004; Sonuga-Barke, 2005).

One theoretical framework (Nigg et al., 2004) captures this heterogeneity in a particularly relevant way with respect to the current series of studies. Relative to other prominent theories which implicated cognitive or motivational differences as a central 
feature of ADHD, Nigg and colleagues' (2004) "multiple pathway model" incorporated temperament constructs in describing the development of ADHD. Temperament is generally defined as a set of early developing traits which are mostly stable across development, consistent across situations, and which are partially heritable (Nigg et al, 2004; Rothbart, 2007). This model (Nigg et al., 2004) focuses particularly on the temperament dimensions of effortful control (i.e., important contributor to self-regulation of cognition and emotion), and positive and negative approach behaviors (i.e., affective and behavioral reactivity). These temperament domains are not posited as alternative measures of the clinical phenotypes; rather, the development of these broad-based and early traits coincide with behavioral and cognitive correlates of ADHD, and provide more developmentally sensitive measures of the emergence of risk for ADHD. Indeed, the temperamental domains emphasized in this model have important influences on the development of attention and self-regulation (Posner \& Rothbart, 2000; Posner, Rothbart, Sheese \& Voelker, 2014). With respect to the current studies, this multiple pathway model (Nigg et al., 2004) describes individual differences in regulatory control and reactivity - which combine and interact with socialization processes - as essential sources of etiological and developmental heterogeneity. As the following literature review makes clear, the current studies are concerned with elucidating whether measures of regulation and reactivity (at the level of the parasympathetic nervous system; PNS) may help clarify documented cognitive heterogeneity in ADHD, as has been suggested in previous research (Ward, Alarcon, Nigg \& Musser, 2015). 


\section{Parasympathetic Nervous System Regulation and Child Psychopathology}

Along with the increasingly well-characterized cognitive and developmental heterogeneity in ADHD (Whalstedt, Thorell, \& Bohlin, 2009; Willcutt et al., 2012) and influential theories positing multiple etiological pathways (Sonuga-Barke, 2002, 2005), there is growing recognition that the developmental differences associated with ADHD involve broad regulatory constructs as well as aspects of executive functioning (Musser et al., 2011; Nigg \& Casey, 2005; Sergeant, 2005). Extant research has increasingly examined the role of self-regulation (e.g., emotion regulation, arousal and effort) across a variety of normative and adverse developmental outcomes using cardiac-derived measures of PNS activity (Beauchaine, 2001; Calkins, Fox, \& Marhsall, 1996; Marcovitch et al., 2010; Miller et al., 2013; Obradovic \& Boyce, 2012; Porges, 2007; Thayer et al., 2009). Among typically developing children, cardiac-derived measures of PNS regulation are linked to differences in cognitive function (Holzman \& Briggett, 2017; Sturge-Apple et al., 2016), including working memory and attention (Gianaros, Van der Veen, \& Jennings, 2004; Pu, Schmeichel \& Demaree, 2010; Suess, Porges, Plude, 1994), as well as emotion regulation (Beauchaine, 2001; Gross, 1998) - two areas that are commonly impacted in ADHD (Graziano \& Garcia, 2016). For this reason, there has been a recent surge in research on ADHD which incorporates measures of PNS regulation.

Much of the work on PNS regulation as an index of self-regulation stems from Porges' $(1995,2007)$ polyvagal theory which proposes that parasympathetic output via the vagus nerve is associated with self-regulation. Polyvagal theory predicts successful broad-based regulation to be associated with the reliable withdrawal of PNS influence 
during challenging states. This is presumed to allow for increases in metabolic resources to cope with challenge (i.e., increased heart rate, respiration, etc.), including cognitive, emotional, or other environmental demands (Porges, 2007). A well-validated index for PNS influence over cardiac activity is respiratory sinus arrhythmia (RSA), which is derived from the dynamic fluctuation in heart rate which occurs naturally during respiration.

The significance of polyvagal theory for developmental psychopathology has been well-recognized through studies which have examined differences in RSA either at rest or in terms of reactivity in response to cognitive tasks and emotion regulation paradigms. In particular, reduced RSA at rest and either blunted or exaggerated withdrawal of RSA during challenging contexts is associated with myriad psychopathology (Beauchaine, 2001; Beauchaine, 2012; Graziano \& Derefinko, 2013). Such patterns of RSA have been posited as a transdiagnostic risk factor for childhood psychopathology (Beauchaine \& Thayer, 2015).

With respect to ADHD, differences in PNS regulation during challenges have been observed across various contexts when compared to non-affected children (Musser et al., 2011, 2018; Rash \& Aguirre-Camacho, 2012; Ward, et al., 2015). For example, in one study, children with ADHD exhibited elevated RSA compared to typically developing youth in the context of and emotion regulation paradigm involving positive and negative emotional stimuli (i.e., consistently elevated PNS response across valence contexts; Musser et al., 2011), and these indices differentiate between heterogeneous behavioral and temperamental profiles among ADHD children (Karalunas et al., 2014; Musser et al., 2013). 


\section{PNS Activity as a Potential Moderator of Working Memory Ability in ADHD}

In addition, and central to the current set of studies, working memory disruptions in ADHD appear to be moderated by PNS dysregulation among children with ADHD (Ward et al., 2015). Working memory refers to a limited capacity, short-term memory which stores, actively updates, and allows manipulation of transitory information (Baeddely, 2007; Engle, 2002; Engle, Tuholski, Laughlin, \& Conway, 1999). In a recent study, children with exaggerated PNS reactivity (i.e., either exacerbated RSA withdrawal or augmentation) and weaker working memory performance were more likely to be diagnosed with ADHD than their peers (Ward et al., 2015). Given the well-established, but heterogeneous association between ADHD and working memory ability cited above, these effects may explain some of the cognitive heterogeneity observed in ADHD. That is, poor working memory was associated with ADHD and its symptoms particularly when compounded by atypical parasympathetic regulation.

These findings suggest an additive process; when working memory deficits are present along with atypical PNS response, ADHD was more likely and more severe (Ward et al., 2015). Critically, however, it remains unclear whether the moderating role of PNS reactivity is specific to working memory only, or whether PNS reactivity relates to other cognitive deficits commonly associated with ADHD, such as response inhibition (i.e., the ability to inhibit a previously habituated response) and response variability (i.e., within-subject inconsistency in reaction time). Specifically, moderation models will examine RSA reactivity during the tests of attention and inhibition as a moderator of the association between each cognitive measure and ADHD (as a diagnosis and in terms of symptom severity). Additionally, replicating the previous research by Ward and 
colleagues (2015) in an ethnically and socioeconomically diverse, clinic-recruited sample will investigate the generalizability of the previously obtained results. This dissertation seeks to address these gaps using a multi-level assessment battery consisting of working memory, response inhibition, and reaction time variability tasks with concurrent assessment of PNS regulation in a diverse, clinical sample of children with ADHD diagnosed using gold-standard assessment procedures.

The current study will contribute to understanding of etiological and executive function heterogeneity in ADHD by further elucidating specific bio-neurocognitive mechanisms, which may serve to influence both prevention and research efforts. Furthermore, a greater understanding of the etiological mechanics of the disorder has the potential to impact clinical practice and pharmacological treatment of the disorder, as new targets may be identified.

\section{Specific Aims and Hypotheses}

Study 1 examines independent and interactive contributions of PNS regulation and working memory in ADHD. Specifically, it is hypothesized that a) working memory (assessed via spatial span) will be weakened in ADHD children compared to typically developing (TD) youths, b) PNS-based regulation during a working memory task (indexed by RSA withdrawal from baseline) will be present for control group, but blunted for the ADHD group, and c) PNS-based regulation will moderate the association between working memory and ADHD, such that blunted PNS-based regulation with weakened WM will predict ADHD (both categorically and continuously). 
Study 2 examines the independent and interactive contributions of PNS regulation and response inhibition, as well as interactions between PNS regulation and attention, in ADHD. The following predictions are hypothesized: a) response inhibition will be weakened in ADHD children compared to typically developing youth, b) vigilant attention performance will be weaker in the ADHD group than in the TD youth group c) PNS-based regulation during a response inhibition task will be present for the control group, but blunted for the ADHD group, d) PNS-based regulation during the task will moderate the association between attention performance and ADHD, such that poor PNSbased regulation with weakened attention will predict ADHD (both categorically and continuously), and e) PNS-based regulation during the task will moderate the association between response inhibition and ADHD, such that poor PNS-based regulation with weakened response inhibition will predict ADHD (both categorically and continuously).

Study 3 will examine the association between reaction time variability and ADHD, as well as the interactive contributions of parasympathetic regulation and reaction time variability in ADHD. Hypotheses include: a) reaction time variability will be greater in ADHD children compared to TD children, b) PNS-based regulation during task will moderate the association between reaction time variability and ADHD, such that poor PNS-based regulation with greater reaction time variability will predict ADHD (both categorically and continuously).

This research will contribute to the field's understanding of how physiological regulation is related to the link between ADHD symptoms and specific cognitive 
difficulties. In addition, significant findings may hold promise for informing future research on etiological pathways, prevention, and individualized treatment approaches. 


\section{CHAPTER II: STUDY 1.}

\section{Introduction to Study 1}

Weak performance on tasks of working memory $(\mathrm{WM})^{1}$ is associated with ADHD diagnoses and symptoms (Martinussen et al., 2005; Rapport et al., 2001;Kasper, Alderson, \& Hudec, 2012). Further, poor WM performance helps to explains variance in associated impairments that are commonly seen with the disorder, such as academic problems (Gropper \& Tannock, 2009). Meta-analytic studies support a relatively robust association between WM - including both auditory and visual/spatial modes - and ADHD diagnosis (Martinussen et al., 2005). However, there is marked heterogeneity in this effect (Kasper et al., 2012), with as many as half of individual children with ADHD exhibiting WM in a normative range (Nigg et al., 2005). In fact, WM is heterogeneous among typically developing children as well, such that the distribution of WM performance in typically developing samples substantially overlaps with ADHD samples (Fair, Bathula, Nikolas, \& Nigg, 2012). That is, while aspects of cognitive functioning (such as reduced performance on WM tasks) are robustly associated with a diagnosis of ADHD, these features are not universal among children with ADHD, nor specific to the disorder (Harvey et al., 2004; Landrø, Stiles, \& Sletvold, 2001).

\footnotetext{
${ }^{1}$ Ward et al., (2015) used the term "short-term memory storage/reordering" to more precisely define the specific aspect of working memory used in that study, based on distinctions drawn from extant empirical work (see Engle et al., 1999 and Conway et al., 2005 for detailed discussions). Here, I use the term "working memory" in keeping with the numerous studies which incorporate similar cognitive tasks.
} 
In line with these empirical developments, contemporary theories propose that there may be multiple developmental profiles (or "pathways") which can manifest as ADHD symptoms (Castellanos, Sonuga-Barke, Milham \& Tannock, 2006; SonugaBarke, 2005; Nigg, Goldsmith \& Sachek, 2004). Following these shifting conceptualizations, recent work has examined how measurement of other broad systems involved in self-regulation may inform the heterogeneous cognitive profiles observed in ADHD. In particular, cardiac-derived indices of parasympathetic nervous system (PNS) activity are sensitive to changes in engagement and attention demands related to performance on a variety of cognitive tasks (Duschek, Muckenthaler, Werner \& Reyes del Paso, 2009; Marcovich et al., 2010; Porges, 2007; Hansen, Johnsen, Sollers, Stenvik \& Thayer, 2004) including tests of WM (Hansen, Johnsen \& Thayer, 2003). In addition, associations between atypical PNS regulation and ADHD have been reported under various contexts, such as during rest (Rash-Aguirre \& Camacho, 2012; Ward et al., 2015), during emotion regulation (Beauchaine et al., 2001; Crowell et al., 2006; Musser et al., 2011), and during challenging cognitive tasks (Ward et al., 2015).

Recent work by our group investigated the contribution of dysregulation in PNS control over heart rate (indexed through respiratory sinus arrhythmia; RSA) and found that exaggerated PNS reactivity (i.e., excessive RSA elevation or withdrawal during the WM task) moderated the association between worse WM ability and greater likelihood of an ADHD diagnosis (Ward et al., 2015). In addition, the combination of atypical PNS response (exaggerated RSA withdrawal) during the task and poor WM performance predicted more severe ADHD symptoms. However, replication of these findings is needed in order to confirm these conclusions and inform future longitudinal research. 
Replication of research findings is a priority in the field of clinical psychological science given that type I error may occur among published research articles much more often than was previously recognized (Ioannidis, 2005, 2012; Maxwell, Lau \& Howard, 2012; Pashler \& Wagenmakers, 2012). Finally, substantiating a cognitive/regulatory profile predictive of ADHD could also inform research on early development and prevention of ADHD.

The current study seeks to replicate the previous study by Ward and colleagues (2015) which examined the moderating role of PNS regulation on the association between WM and ADHD. One of the limitations of that study was the relative ethnic homogeneity of the samples utilized; for example, over $80 \%$ of the overall sample identifying as non-Hispanic Caucasian. The present study expands on Ward et al. (2015) by examining these associations in an ethnically diverse, clinically-recruited sample of children. Generally, it is expected that results will mirror those of Ward et al. (2015). Specifically, it is hypothesized that 1) WM will be weakened in ADHD children compared to typically developing youths, 2) PNS activity indexed by respiratory sinus arrhythmia (RSA) at rest will be elevated in children with ADHD relative to controls 3) PNS-based regulation during the WM task (indexed by RSA withdrawal during the task) will be present for control group, but blunted for the ADHD group, and 4) PNS-based regulation will moderate the association between WM and ADHD, such that blunted PNS-based regulation with weakened WM will predict ADHD (both categorically and continuously). 


\section{Study 1 Methods}

\section{Participants}

Participants were 196 children aged 7 to 12 years $(M=9.27, S D=1.41) ; 126$ met DSM 5 criteria for ADHD (80\% male; 65\% combined presentation, $23 \%$ inattentive presentation, $12 \%$ hyperactive/impulsive presentation), and 70 were typically developing (TD) comparison youth ( $57 \%$ male). Children younger than age 12 were recruited given the age criterion for a diagnosis of $\mathrm{ADHD}$, which requires onset of symptoms prior to age 12 years (American Psychiatric Association, 2013). Thus, children in the ADHD group are likely to be experiencing current and ongoing impairment and high symptom severity. Additionally, the age range of 7-12 years old was utilized for Study 1 specifically to mirror the age range utilized by Ward et al. (2015). Racial and ethnic minority children (identifying as Hispanic/Latino or racial minority) made up a large proportion of the sample (92.1\% of controls, $94.4 \%$ of ADHD). Specifically, approximately $15 \%$ of participants (23\% of controls, $12 \%$ of ADHD) were identified by a parent as a racial minority (10\% African American, 1.2\% Asian, and 1\% American Indian, 1.9\% other/mixed race). Further, $89 \%$ of the overall sample identified as Hispanic (i.e., an individual can fall under both categories). Additional demographic and diagnostic details are provided in Table 1. The local Institutional Review Board approved the study; all procedures conformed to the Ethical Principles of Psychologists and Code of Conduct (American Psychological Association, 2002). Parents provided written informed consent and children provided written informed assent. 


\section{Procedures}

Recruitment. Children with ADHD in the current study are a subset of children enrolled in a double-blind, cross-over study examining tolerance to stimulant medication in children with ADHD. The larger study took place in the context of a Summer Treatment Program (STP) over the course of three years. Families in the larger study were recruited through several sources, including a university treatment center, referrals from schools and physicians, and advertisements (billboards, newspaper, radio). Typically developing (TD) children were recruited from the community through advertisements (e.g., flyers) and community exhibitions.

Exclusion Criteria. All participants in the larger study were required to meet DSM 5 criteria for ADHD and to have no documented contraindication for use of methylphenidate, documented intolerance to methylphenidate, or failed trial of OROS methylphenidate at full therapeutic dose. Children in the TD group were required to have no more than three ADHD symptoms endorsed by parents. In addition, both ADHD and TD youth were required to meet the following inclusion criteria for the current study: Full Scale IQ > 75, no diagnosis of autism, seizures, arrhythmias, hypertension, Tourette's or Tic disorders, psychoses, mania, or other disorders made worse by stimulants. Other disorders, including disruptive behavior and/or mood or anxiety disorders were free to vary and treated as covariates as appropriate in relevant analyses.

Diagnostic Procedures and Measures of Psychopathology. Participants in both groups (ADHD and TD) underwent a multi-gate screening process to establish eligibility and diagnostic assignment. A parent/guardian completed questionnaires and standardized 
rating forms (see Procedures), as well as the Diagnostic Interview Schedule- Child Version (DISC-IV; Shaffer et al., 2000), with a trained master's-level clinician. Additionally, parent-report and teacher-report on the Disruptive Behavior Disorders Rating Scale (DBD-RS; Pelham, Gnagy, Greenslade, and Milich, 1992) and the Pittsburgh Modified Conners Rating Scale (Pelham, Fabiano, \& Masseti, 2005) were obtained for all children with ADHD. However, only parent-report (i.e., no teacherreport) for the DBD-RS and Conners was obtained for TD children. All children (ADHD and TD) completed the Wechsler Abbreviated Scale of Intelligence (WASI-II; Wechsler, 2011) and the word reading, spelling, and numerical operations sub-tests of the Wechsler Individual Achievement Test (WIAT-III; Wechsler, 2009).

Diagnoses of ADHD were made according to best-practice recommendations (Pelham, Fabiano, and Massetti, 2005) and included parent and teacher ratings of ADHD symptoms (from the DBD-RS), a structured parent interview (DISC-IV), and parent and teacher ratings on the Impairment Rating Scale. Two Ph.D. level clinicians reviewed all available data from the initial assessment to confer diagnoses of ADHD and disruptive behavior disorders (i.e., CD and/or ODD) and ensure children met inclusion criteria. If disagreements occurred (less than $1 \%$ of cases), a third clinician reviewed the file and majority decision was used. For the purposes of this study, all other comorbid diagnoses (excluding ODD and CD) were determined based on endorsements of parent report on the DISC-IV (Shaffer et al., 2000).

Medication Status and Context. The larger study utilized a crossover design in which children were given placebo for either the first or second half of the STP (3 weeks 
each condition) after an initial 2-week titration period. All data used in the current study, for the ADHD sample, were obtained after children were "washed out" of stimulant medications (i.e., at least 48 hours without medication; equivalent to 7 half-lives) and currently taking placebo.

\section{Measures}

Psychopathology. Parent-reported symptoms of ADHD and oppositional defiant disorder were measured by the DBD-RS (detailed above). The mean rating of DSM-5 ADHD symptoms was utilized as a continuous measure of ADHD severity. Mean symptom rating was selected rather than the count of endorsed symptoms because children in the ADHD group needed to have at least six endorsed symptoms in either or both the inattention or hyperactive/impulsive symptom domain. Therefore, using symptom count would result in a greater restriction of range in this variable. A structured interview with the child's parent (DISC-IV; Shaffer et al., 2000) was also administered. Comorbid diagnoses of ODD, CD, anxiety, or mood disorders were measured using parent endorsements on the DISC-IV for the respective disorder.

Spatial Span. The Spatial Span task (similar to the CANTAB Spatial Span; Fray, Robbins \& Sahakian, 1996) backward condition was used to assess visual-spatial shortterm memory (i.e., re-ordering and rehearsal of visual-spatial information). The task was administered using E-prime software. The task stimuli are presented as a series of white boxes arranged on-screen in fixed locations. The boxes light-up one at a time, followed by a tone, after which participants click on the boxes in the reversed order of what was displayed. Before beginning the automated task, children demonstrate comprehension by 
correctly completing a single practice trial (span of three); no children in the current sample failed to advance to testing. During the task, two trials are administered at each span length (i.e., a "block"), beginning with a span length of three (i.e., three boxes light up sequentially) and continuing up to a maximum span of nine boxes. One element is added to the span in each subsequent block if at least one trial was completed with $100 \%$ accuracy. The task is automatically ended when at least one error is produced on both trials within a block.

Analyses utilize a partial credit scoring algorithm (Conway et al., 2005) which assigns credit according to the proportion of correctly-recalled items within a trial. In addition, participant scores on the Spatial Span were "load-weighted," meaning that it is possible for each item in a span to result in a single point (e.g., a span of three has a maximum score of three, while a span of five has a max score of five). The result is greater distinction according to performance, wherein individuals who reach larger span lengths and more consistent performance across trials may obtain a higher score. It should be noted that while this approach is considered best practice by many, it also is highly correlated with "unit weighted" and "all-or-nothing" approaches which are also commonly utilized extant cognitive literature ( $r=.87$ to .93 ; Conway et al., 2005).

Physiological Recording. Disposable silver/silver-chloride electrodes were placed in a standard electrocardiogram (ECG) and impedance cardiography (ICG) configuration. The ECG electrodes were placed at the right collar bone and the tenth-left rib with a ground electrode placed at the tenth-right rib. To estimate respiration rates, ICG was used with two voltage electrodes placed below the suprasternal notch and xiphoid process and 
two current electrodes placed along the spine 3 to $4 \mathrm{~cm}$ above and below the voltage electrodes. ECG and ICG were recorded continuously during a two-minute resting baseline, a neutral baseline, and the WM task (each detailed below). The R-R series was sampled at 1,000 Hz. Heart rate, interbeat-interval (IBI) and respiration rate data were derived using the ECG and ICG data following collection.

Respiratory Sinus Arrhythmia (RSA). The RSA was indexed by extracting the high frequency component $(>0.15 \mathrm{~Hz})$ of the R-R peak time series. Respiratory sinus arrhythmia was derived using spectral analysis of the R-R time series (Berntson, et al., 1997), in 30 second epochs across rest, neutral baseline, and Spatial Span task. A length of 30 seconds was utilized given that several participants completed the spatial span task in less than 60 seconds. The R-R time series data were detrended and Fast Fourier transformation was applied. The obtained high frequency band of heart-rate variability data was set over the respiratory frequency band of 0.24 to $1.040 \mathrm{~Hz}$. Respiration rates and amplitudes were derived from the impedance cardiograph signal (Z0).

The R-R waves were examined for artifacts and outliers using MindWare® Heart Rate Variability software, version 3.1 (MindWare, 2012). Artifacts were removed by trained research assistants using the software and visual inspection. This process was repeated by independent raters on a randomly selected sub-sample consisting of $20 \%$ of the cases form the complete sample, and satisfactory inter-rater reliability was indicated (all $k>0.85$ ). In addition, there were no between-group differences in the artifacts identified, all $p>0.50$. 
Resting and Neutral Physiological Baselines. Prior to physiological recording, children's resting RSA was assessed during a single two-minute period while in a still, seated position. Additionally, children completed a neutral period task before and after the Spatial Span task. This neutral period lasted approximately two minutes and included viewing 10 pictures from the neutral set of the International Affective Picture System (Lang, Bradley \& Cuthbert, 1997) on a computer screen. The purpose of the neutral period was to account for the physiological response associated with orienting and attending, as well as facilitating the return of physiological activity to baseline levels prior to beginning the next task. Respiratory sinus arrhythmia during this neutral baseline task was used to calculate physiology change scores.

Physiological Reactivity. Physiological reactivity to task demands were calculated by subtracting average RSA during the first $30 \mathrm{sec}$ epoch of the neutral baseline condition from average RSA during the first $30 \mathrm{sec}$ epoch of the WM task (described below).

$$
\text { RSA [Spatial Span] - RSA [Neutral period] }=\Delta \text { RSA [reactivity }]
$$

RSA during the task was compared to the neutral period rather than the resting baseline in order to avoid indexing reactivity solely associated with orienting and responding to the task.

\section{Study 1 Analytic Plan.}

Group differences (i.e., ADHD vs. control) in WM performance, resting RSA, and RSA reactivity during the WM task (i.e., RSA change scores calculated as on-task RSA minus neutral period RSA) were each examined using analysis of covariance (ANCOVA). Separate linear regression models were used to examine continuous 
associations between ADHD symptom severity and each of the following predictors: WM performance; resting RSA; RSA reactivity during the WM task. Additionally, regression-based moderation analyses were used to examine the interaction of RSA reactivity and WM performance in predicting ADHD (as both a diagnostic category and continuous dimension of symptom severity, in separate models) using the PROCESS macro (Hayes, 2013). Variables were mean-centered prior to moderation analyses. The PROCESS macro performs both linear- and logistic-regression based moderation analyses, probes interaction effects, and provides multiple indices of conditional effects. Pick-a-point effects are given for logistic moderation models, while Johnson-Neyman conditional effects are presented for linear regression moderation models (Bauer \& Curran, 2005; Hayes, 2013). Missing data were addressed through list-wise deletion for all models.

\section{Study 1 Results}

\section{Distribution and Power Analysis}

When examining the entire sample (i.e., both youth with and without ADHD), mean scores from the DBD Rating Scale demonstrated kurtosis $(z>1.96)$, but not skew. This was expected given the inclusion of a control group exhibiting few symptoms of ADHD by design. Thus, as planned, linear and logistic regression-based approaches were utilized given their relative robustness against violations of normality (Cohen, Cohen, West \& Aiken, 2003). The mean raw scores of parent-rated ADHD symptoms across the two domains measured by the DBD-RS (DSM-5 inattentive and hyperactive/impulsive domains) were highly correlated with one another $(r=.81)$, as well as with the overall 
ADHD symptom ratings (both $r>$.95). Using G*Power (Faul, Buchner, Lang, 2009), post-hoc power analysis of ANCOVA for sample size $\mathrm{N}=196$ indicated power of .74 (borderline) to detect a small effect $\left(\right.$ partial- $\left.\eta^{2}=0.06\right)$, consistent with effects sizes obtained for differences in WM and RSA in a previous study of children with ADHD (partial- $\eta^{2}$ 's $=.06$ and .03 , respectively; Ward et al., 2015). For the same sample, post-hoc power analysis for logistic regression indicated power of .80 (satisfactory) to detect a medium effect size (odds ratio $=1.70)$ and, for linear regression, power of .81 (satisfactory)to detect a small effect $\left(R^{2}=.03\right)$. These proposed effects sizes are consistent with those found in previous research (Ward et al., 2015).

\section{Sample Characteristics}

Table 1 provides demographic and clinical description of the sample by group. Compared to children in the control group, children with ADHD were more likely to be male $\left(\chi^{2}=11.81, p<.001\right)$ and less often belonged to a racial minority $\left(\chi^{2}=3.69, p<\right.$ .05). However, racial minority individuals were not significantly different with regard to WM, resting or task RSA, RSA-change, nor ADHD symptom severity (all $p>.21$ ). Finally, no significant between-group differences were observed in terms of age, estimated full scale IQ, or proportion of Hispanic/Latino (all $p>.10$ ).

As expected regarding clinical characteristics, children with ADHD had more symptoms of oppositional defiant disorder (ODD) and conduct disorder (both $p<.001$ ), were more likely to have a diagnosis of those disorders (both $p<.05$ ), and were more often prescribed medication for ADHD prior to entering the study (i.e., no subjects in the control group were medicated; $p<.001)$. These differences are largely representative of 
children with ADHD in the general population (APA, 2013). No statistically significant between-group differences were observed in diagnoses of anxiety or depressive disorders (all $p>.05)$.

Correlations of the primary variables of interest, demographic, and clinical characteristics are displayed in Table 2. Child age was significantly correlated with WM performance, and RSA during Spatial Span (i.e., not reactivity). Child Full Scale IQ was significantly correlated with WM performance $(p<.05)$. Given these significant associations, as well as theoretical links with the primary variables of interest and consideration of the covariates included in the original manuscript (Ward et al., 2015), age, gender, previous medication status, and ODD symptom count were included as covariates in all models. These are the same covariates included in models examined by Ward et al., (2015). Although IQ was associated with WM task performance, experts have criticized using IQ as a covariate in studies of cognitive functioning and ADHD (e.g., Dennis et al., 2009).

\section{Analyses Using ADHD Diagnosis}

Analyses initially focused on the ADHD syndrome as defined by DSM-5 criteria (American Psychiatric Association, 2013).

Working Memory according to ADHD Diagnosis. A one-way ANCOVA examined group differences in WM while controlling for covariates. The main effect of diagnostic group on WM was not significant, $F(6,175)=0.00, p=.99$.

Baseline RSA according to ADHD Diagnosis. At resting baseline, youth with $\operatorname{ADHD}(E M M=6.17, S E=0.13)$ exhibited significantly lower resting RSA (i.e., an 
indication of reduced PNS-based regulation) compared to control youth (EMM=7.09, $S E=0.22), F(6,179)=9.59, p<.01, \eta_{p}^{2}=.05$. Youth with $\operatorname{ADHD}(E M M=6.25, S E=0.14)$ also exhibited significantly lower RSA during the neutral baseline condition compared to control youth $(E M M=6.90, S E=0.23), \mathrm{F}(6,177)=4.51, p<.05, \eta_{p}{ }^{2}=.03$. Between-group RSA effects at baseline are shown in Table 3.

Autonomic Reactivity Effects according to ADHD Diagnosis. Respiratory sinus arrhythmia reactivity was indexed by subtracting the respective RSA values during the neutral baseline condition (see Methods) from the RSA value during the WM task. ANCOVA revealed no significant between-groups difference in RSA reactivity during the WM task $(\mathrm{F}(6,177)=0.88, p>.34$ as summarized in Table 3a.

Test of Moderation Effects. To test the hypothesis that the association between ADHD diagnosis and WM performance would be moderated by RSA reactivity, moderation analyses were conducted using a logistic regression approach (via PROCESS; Hayes, 2013). When predicting diagnostic group status (i.e., ADHD vs typically developing), the interaction of mean WM score and RSA reactivity was not statistically significant $(z=0.21, b=0.64, p>.80)$, indicating that moderation was not present.

\section{Analyses Using Continuous/Dimensional ADHD Measures}

Multiple regression analyses examined the association between WM performance as the predictor variable and overall ADHD severity as the outcome variable, which was measured using the mean of parents' ratings (i.e., raw scores) for ADHD symptoms on the DBD-RS (Pelham et al., 1992). To investigate DSM-5 dimensional specificity, mean raw scores for inattentive and hyperactive/impulsive items (also from the DBD-RS) were 
used to index inattentive and hyperactive/impulsive symptom severity as the outcome in separate models. Child age, sex, ODD symptoms, and medication status were treated as covariates in all models described below.

Working Memory and Continuous Measures of ADHD. In the model with mean overall ADHD symptom rating as the dependent variable, WM score was not significantly associated with overall ADHD rating, $\beta=-0.06, t(174)=-1.10, p>.27$ (Table 4a). Additionally, WM score was not significantly associated with ratings of the inattentive symptom domain, $\beta=-0.05, t(175)=-1.19, p>.23$, nor was the relationship significant between $\mathrm{WM}$ and hyperactive/impulsive symptom ratings, $\beta=0.01, t(175)=$ $0.13, p>.89$ (see Tables 4b, 4c).

At-Rest RSA and Continuous Measures of ADHD. Results of regression analyses are summarized in Table 4a. The model with overall ADHD rating as the outcome variable and resting baseline RSA and all covariates as predictors was significant, $F(5$, $178)=54.97, p<.001, R^{2}=.61$. Specifically, RSA at resting baseline was significant and negatively associated with overall ADHD symptom rating, $\beta=-.16, t(175)=-3.27, p<$ $.01, s r^{2}=.023$. In separate model, RSA during neutral baseline fell short of significant as a predictor of overall ADHD rating, $\beta=-.10, t(176)=-1.95, p=.05, s r^{2}=.008$. Lower resting RSA predicts greater ADHD severity, indicating that children in the ADHD group tend to exhibit reduced PNS-regulation over heart rate.

To examine the specificity of significant findings with respect to DSM-5 ADHD symptom dimensions, multiple regression analyses replicated these analyses with resting RSA as the predictor of interest, and inattentive or hyperactive/impulsive symptom 
ratings as the outcome (hyperactive/impulsive or inattentive symptom severity, respectively, was added as an additional covariate). Resting RSA was a significant negative predictor of inattention, $\beta=-0.16, t(176)=-4.04, p<.001, s r^{2}=.026$, but not hyperactivity/impulsivity ( $p=.27$; see Tables $4 \mathrm{~b}$ and $4 \mathrm{c}$ ).

Autonomic Reactivity and Continuous Measures of ADHD. Testing the hypothesis that autonomic reactivity during the WM task (i.e., change in RSA from neutral period) would be related to ADHD severity, the model with RSA change plus covariates was significant, $F(5,176)=50.20, p<.001, R^{2}=.59$. However, RSA change was not a significant predictor $(\beta=.07, t(176)=1.39, p=.16)$ when accounting for covariates. When examining potential specificity with respect to ADHD symptom domains (similar to with resting RSA above), RSA reactivity was not a significant predictor of inattentive severity $(p=.44)$ nor hyperactive/impulsive severity $(p=.66)$.

\section{Test of Moderation Effects on Continuous Measures of ADHD. Moderation}

analyses were conducted using mean ADHD symptom rating as the continuous outcome variable. The models were identical to the moderation models described above which used ADHD diagnosis, but with symptom severity (from the DBD-RS) as the outcome variable and using a linear regression approach. The interaction of WM performance and RSA reactivity was significant in predicting overall ADHD symptom severity, $b=-0.81$, $S E=0.31, t(170)=-2.63, p<.01$. Conditional effects are displayed in Table 5 and indicate that lower WM score was associated with higher mean symptom rating when RSA withdrawal was above average, but not at mean levels of RSA withdrawal. 
Additional moderation analyses examined the WM - RSA interaction effect with individual ADHD symptom domains (i.e., either inattentive or hyperactive/impulsive symptom ratings, separately) as the dependent variable, adding the other respective domain as a covariate. Neither the model predicting inattentive symptom ratings $(p=.14)$ nor hyperactive/impulsive symptom ratings $(p=.32)$ was significant.

Follow-up analyses examined the moderating effect of RSA reactivity among the ADHD subgroup only $(\mathrm{n}=126)$. The interaction was significant $b=-0.67, S E=0.28$, $t(114)=-2.35, p<.05$, and indicated similar conditional effects to the same model in the full sample. Children who displayed both weaker WM as well as excessive RSA withdrawal were predicted to have the most severe ADHD symptoms.

\section{Study 1 Discussion}

Prevailing etiological theories of ADHD implicate deficits in cognitive constructs, such as working memory (WM), as primary drivers of ADHD symptoms and impairments (Barkley, 1997; Rapport et al., 2001), but youth with ADHD are heterogeneous with respect to individual decrements in these constructs (Kasper et al., 2012; Nigg et al., 2005; Wahlstedt, Thorell, \& Bohlin, 2009). Prior work by our group has demonstrated that heterogeneity in WM among community-recruited children with ADHD is accounted for by the co-occurrence of abnormal PNS-based regulation (Ward et al., 2015). The current study sought to replicate and extend these findings by examining these relationships in a more ethnically diverse, clinic-recruited sample.

The first hypothesis that children with ADHD would experience reduced WM relative to TD children was not supported. These findings run counter to previous 
literature, as well as our previous study, which reported significantly reduced WM performance (partial-eta ${ }^{2}=.03$ to .06 ) among children with ADHD compared to typically developing children (Ward et al.,2015). However, while recent meta-analyses yield large effect sizes for WM differences among ADHD youth, these studies also reveal statistically significant heterogeneity statistics (Kasper et al., 2012; Martinussen et al., 2005; Willcutt et al., 2005) indicating that inconsistency in the effects are not likely due to chance (Higgins, Thompson, Deeks, Altman, 2003). The recent meta-analysis by Kasper and colleagues (2012) noted that considerable between-study variability in working memory performance among youth with ADHD is moderated by the type of memory task utilized. Specifically, WM tasks with more intense demands of the central executive component of WM have been shown to be associated more consistently with impaired performance among ADHD samples than tasks which emphasize recall and reordering of memorized items (such as the span task utilized here). The current findings may be emblematic of this variability, which would suggest group differences in WM may be present if assessed through a more comprehensive battery. At the same time, PNS-based regulation has also been found to impact cognitive performance in children with and without ADHD (Marcovitch et al., 2010; Suess, Porges, Plude, 1994; Ward et al., 2015). The current study is concerned with investigating the role of PNS regulation in this well-documented heterogeneity and, therefore, we examine these associations below.

Both attenuated PNS-based regulation, as well as blunted PNS reactivity during challenging tasks, are associated with psychopathology in children, including among youth with ADHD (Beauchaine, 2001; Graziano \& Derefinko, 2013; Musser et al., 2011). Additionally, low baseline PNS-based regulation is associated with reduced WM 
performance in children and adults (Gianaros et al., 2004; Hansen, Johnson \& Thayer, 2003). In the present study, children with ADHD exhibited significantly reduced RSA (a cardiac index of PNS regulation) at rest when compared to children in the TD group. Further, when ADHD symptoms were examined continuously, reduced RSA at rest was associated with greater ADHD severity. Specifically, low resting RSA was specifically associated with higher parent ratings of inattention, but not hyperactivity. These results suggest reduced PNS regulation is more common among ADHD youth than TD children and that children with greater inattention may be particularly characterized by reduced PNS. These findings corroborate other research demonstrating that reduced tonic PNS influence over heart-rate is associated with myriad psychopathology (Beauchaine, 2001), including ADHD (Rash \& Aguirre-Camacho, 2012). Notably, however, these findings differ from those of Ward et al. (2015), where RSA at rest was significantly higher among youth with ADHD than the TD group. While prevailing theoretical conceptualizations suggest children with ADHD will exhibit reduced vagal-mediated PNS influence over resting heart rate (i.e., reduced resting RSA; Beauchaine \& Thayer, 2015; Porges, 2007), results have been mixed. A recent systematic review of six studies concluded reduced heart-rate variability (a measure of PNS regulation related to RSA) at baseline may characterize children with ADHD (Rash \& Aguirre-Camacho, 2012). A more recent meta-analytic study (of six studies of children; Koenig et al., 2017) concluded no significant differences in heart rate variability between ADHD children and TD youth. Importantly, both reviews were unable to examine moderators, such as age and/or gender, due to a small number of extant studies. However, reports of reduced baseline PNS-regulation are often linked to samples with co-occurring externalizing 
symptoms (Beauchaine et al., 2001; Crowell et al., 2006; Hinnant \& El-Shiekh, 2009), which were substantially more prevalent in the current clinic-referred sample (Table 1) relative to our previous study (Ward et al., 2015). Although our analyses controlled for the presence of ODD symptoms, this is an imperfect means of statistically controlling for the full gamut of externalizing psychopathology; and it may be that ADHD symptoms and externalizing problems are each associated with atypical PNS-based responding, but in different ways, thereby influencing the current findings. Further, previous studies examining externalizing comorbidity have noted reduced PNS-based regulation at-rest (Hinnant \& El-Sheikh, 2009) or during tasks (Tenenbaum et al., 2017), as well as blunted reactivity when engaged in challenging tasks (Beauchaine et al., 2001; Musser et al., 2013).

Parasympathetic nervous system reactivity during the WM task was also reexamined here. Using change in RSA (from neutral task to the WM task) to index PNS reactivity, between-group differences in PNS reactivity were not significant. This was somewhat surprising given the significant correlation between RSA-change and ADHD severity (Table 2). In addition, comparing mean level of RSA during resting and task periods for each group (Table 3 and Figure 2) suggests that mean RSA among the ADHD group increased across these periods while mean RSA among TD subjects exhibited less change. However, non-significant differences in PNS reactivity to the WM task are consistent with the findings by Ward et al. (2015). Given the observed differences in PNS-based regulation, and the previously documented relationship between PNS-based regulation and cognitive function (Gianaros et al., 2004; Hansen, Johnson \& Thayer, 2003; Holtzman \& Briggett, 2017), we continued to pursue the question of whether 
heterogeneity in WM among ADHD children can be explained by PNS reactivity, as observed previously (Ward et al., 2015).

The interaction of WM performance and RSA reactivity was significant in predicting ADHD symptom severity. The current finding was corroborated by a similar significant interaction when analyzed in the ADHD group only, but a non-significant interaction effect among the control group. Conditional effects indicated that, for those children with an exaggerated PNS response during the task (i.e., greater elevations in RSA from neutral to WM task periods), lower WM score predicted higher ADHD ratings. However, when PNS reactivity was within approximately 1 SD of the sample mean, the link between WM and ADHD severity was not significant. Of note, the moderation model which examined the interaction of PNS reactivity and WM with dichotomous ADHD diagnosis as the outcome variable was not significant. This finding diverges from the results obtained by Ward and colleagues (2015) where the moderation model was significant both when ADHD was considered in terms of continuous severity and as a dichotomous category. The precise reason is unclear but may be illustrative of the differences between the Ward et al (2015) sample and the current sample, with the latter exhibiting substantially higher rates of comorbid externalizing disorders and ethnic diversity. As discussed previously, PNS reactivity to challenging contexts does vary as a function of externalizing symptoms independent of ADHD (Beauchaine, Gatzke-Kopp \& Mead, 2007; Beauchaine, Hinshaw \& Pang, 2010; Beauchaine, 2012).

These results replicate previous findings wherein PNS reactivity outside of an optimal range combined with weakened WM performance predicted more severe ADHD symptomatology (Ward et al., 2015). Children with ADHD exhibit differences in 
autonomic functioning in a variety of contexts including emotion regulation (Musser et al., 2011, 2013), cognitive tasks (Borger et al., 1999; Ward et al., 2015), and at rest (Negrao, Bipath, Van der Westhuizen, Viljoen, 2011; Ward et al., 2015). In theory, PNS regulation (indexed by RSA) is one facet of broader self-regulation system which includes interconnectivity between the vagus nerve, brainstem nuclei (e.g., nucleus ambiguus), and prefrontal cortical networks (Porges, 2007; Thayer, Hansen, Saus-Rose \& Johnsen, 2009). It may be that some ADHD children experience inefficient operating in this "central-autonomic network" (Thayer et al., 2009) as partially indexed here through RSA (Holtzman \& Briggett, 2017). This would lead to inefficient regulation of attention, social behavior, and cognitive functioning, leading to the behavioral and cognitive problems that characterize ADHD. Such a viewpoint overlaps with models of ADHD which frame optimal self-regulation as consisting of both bottom-up (i.e., autonomic and sub-cortical) and top-down (i.e., cortically-mediated executive functions) capacities (Nigg, Goldsmith, Sachek, 2004; Rothbart, Derryberry \& Posner, 1994). Under such a model, WM ability associated with top-down self-regulation can be hampered by abnormal bottom-up regulation which may include PNS responding (Porges, 2007; Ward et al., 2015).

Overall, it is concluded that PNS regulation is a promising mechanism for explaining heterogeneity in WM performance among children with ADHD. These findings may also align with multi-pathway etiological models of ADHD, which propose that disrupted temperamental regulation and reactivity play a pivotal role in the development of ADHD and eternalizing symptoms, and which are partially indexed through cardiac-based PNS measures (Nigg et al,. 2004; Calkins \& Keene, 2004). The 
current findings are significant in that they replicate previous findings within a sample that varied considerably in terms of ethnic diversity and referral source. For example, 93\% of the current sample identified as belonging to an ethnic minority group (primarily Hispanic), which is in stark contrast to the prior study which was majority Caucasian; additionally, the current sample recruited treatment-seeking subjects while the previous study utilized a community-recruited sample (Ward et al., 2015).

Despite the numerous strengths of the present study, including the large sample, well-characterized diagnostic groups, and multi-method approach, there are limitations. First, the study is cross-sectional and, therefore, it is unclear how PNS regulation and WM problems interact across development, whether one precedes the other, or if they are both preceded by a third causal variable. Secondly, while the current study used a measure of spatial WM which is well-represented in findings linking working memory deficits to ADHD, a more comprehensive battery would allow elucidation of multiple aspects of working memory. For example, a battery of WM tasks tapping auditory/verbal working memory as well as visual-spatial modes, or multiple tasks which tap the various components of WM (e.g., storage/rehearsal, central executive, etc.) to varying degrees. Finally, sympathetic nervous system responding is also an essential factor in the central autonomic network described above. Measuring the impact of sympathetic activity at baseline and in response to cognitive challenge is warranted, and currently in progress within our group. Future studies that address these limitations through use of longitudinal case-control designs and more comprehensive batteries will be well-positioned to describe the developmental trends which distinguish the role of WM and autonomic function in heterogeneous ADHD etiology. 
These results indicate a promising initial step in describing ADHD in terms of underlying cognitive and physiologic functioning, while further establishing that such a description is likely not found at a single level of analysis (Castellanos et al., 2006; Sonuga-Barke, 2005). More specifically, the results suggest that ADHD is likely more severe when weakened cognitive functions are present alongside compromised physiological regulation. These findings set the stage for future work examining the role of PNS regulation and reactivity as a modifier of cognitive development regarding ADHD and other psychopathology. 


\section{CHAPTER III: STUDY 2.}

\section{Introduction to Study 2}

A deficit in response inhibition has been proposed as a central feature underlying ADHD (Barkley, 1997) and has been shown to be associated with many of the characteristics of the disorder (Lijffijt, Kenemans, Verbaten \& van England, 2005; Nigg, 1999; Walcott \& Landau, 2004). Paradigms for objectively measuring response inhibition include computerized tasks, such as the Go/No-Go task, wherein a child must respond as quickly and accurately to target stimuli as possible, while also inhibiting responses to similar, less frequent non-target stimuli (Trommer, Hoeppner, Lorber \& Armstrong, 1988). Increased errors of commission (i.e., responses to non-target trials) indicate disinhibition or impulsive responding, while errors of omission (i.e., failures to respond to target stimuli) are interpreted as an indicator of inattention (Trommer et al., 1988;

Metin, Roeyers, Wiersema, van der Meere \& Sonuga-Barke, 2012). Additionally, slowed reaction times to stimuli indicate difficulty with response execution (Borger \& van der Meere, 2000; Tillman, Thorell, Brocki \& Bohlin, 2007). Overall, a large existing literature indicates that children with ADHD tend to exhibit slower reaction times and commit more errors of both kinds on response inhibition tasks, such as the Go/No-Go (Casey et al., 1997; Lijffijt, Kenemans, Verbaten \& Engeland, 2005; Slaats-Willemse, Swaab-Barneveld, Sonneville, van der Meulen \& Buitelaar, 2003; Sonuga-Barke, Bitsakou \& Thompson, 2010; Trommer et al., 1988). At the same time, deficits in response inhibition are not universal among children diagnosed with ADHD; that is, similar to findings regarding WM, there is substantial heterogeneity in terms of accuracy 
and reaction times on response inhibition tasks among youth with ADHD (Nigg et al., 2005; Sonuga-Barke et al., 2010). Further, there is also wide heterogeneity observable in the performance on these tasks among typically developing youth (Fair et al., 2012).

Recent etiological theories reconcile with these findings by positing multiple developmental pathways to the ADHD diagnosis (Nigg et al. , 2004; Sonuga-Barke, 2005). These pathways likely involve the interaction of multiple substrates (or risk factors) which are often studied in isolation (Nigg, 2010; Castellano et al., 2006). As mentioned previously, recent work has examined how ADHD may be associated with PNS activity at rest (Rash \& Aguirre-Camacho, 2012; Tenenbaum et al., 2017; Ward et al., 2015) and dynamic PNS response during challenging cognitive tasks (Crowell et al., 2006) as well as during emotion regulation paradigm (Musser et al., 2011). Furthermore, it appears that better performance on cognitive tasks is related to an optimal range of RSA reactivity (i.e. PNS response) such that too little or too much RSA withdrawal during a task is associated with worse performance on that task (Marcovitch et al. 2010). Additionally, findings from Ward and colleagues (2015) and the current results from Study 1 have shown that 1) ADHD children tend to exhibit PNS dysregulation at baseline and 2) abnormally strong PNS responses to a task predict a strong link between shortterm memory problems and greater ADHD severity. However, it remains unclear whether this profile of weakened cognitive and self-dysregulation may help explain betweensubject heterogeneity in other commonly identified cognitive correlates of ADHD, such as response inhibition decrements. 
The current study examines between-group effects of performance on a Go/NoGo task (GNG) using a case-control design in sample of children with well-characterized ADHD diagnoses and control subjects. In addition, the moderating effect of PNS reactivity on the association between ADHD symptoms severity (as a continuous outcome) and GNG performance metrics is investigated. Specifically, it is hypothesized that a) response disinhibition metrics (GNG errors of omission and errors of commission) will be elevated in ADHD children compared to typically developing youth, b) PNSbased regulation during a response inhibition task will be blunted for the ADHD group when compared to the control group, and c) PNS-based regulation during the task will moderate the association between GNG errors (i.e., both Error types) and ADHD, such that poor PNS-based regulation with weakened response inhibition will predict ADHD.

\section{Study 2 Methods}

\section{Summary}

Whereas the design of Study 1 was aimed at replicating a previous study, Study 2 aims to further investigate whether a similar overall relationship is observed when other areas of executive functioning are considered. Namely, a Go/No-go task (GNG) was completed by children both with and without ADHD while ECG data were recorded. The GNG task yields indices of response inhibition, response execution, and vigilant attention. In Study 1, participant age range was restricted to 7-12 years old to resemble the original study being replicated. However, replication was not an express goal for Study 2, and so 6-year-old children were included in Study 2 (i.e., the age range of subjects is 6 to 12 years old). This age is inclusive of the period in which ADHD is often 
identified (APA, 2013) and response inhibition has already begun to develop (Bedard et al., 2002; Rothbart, Derryberry \& Posner, 1994; Williams et al., 1999). Therefore, this age range was deemed reasonable for Study 2.

The primary analytic aims of Study 2 include between-groups analysis (i.e., ADHD-diagnosed and typically-developing children) of these GNG measures, as well as multiple regression to examine associations between GNG measures and ADHD symptom severity (i.e., a continuous measure of ADHD psychopathology). In addition, as in Study 1, ECG data were used to derive cardiac-based indices of RSA, an index of PNS influence over heartrate. A between-group comparison of resting RSA is examined briefly to ensure a profile consistent with samples from Study 1. However, the primary analyses involve regression-based moderation models which examine the potential moderating role of RSA-change (an index of PNS reactivity) on the association between GNG performance and ADHD (both as a diagnosis and as a continuous dimension of symptom severity).

\section{Participants}

For Study 2, participant ages ranged from 6 to 12 years $(\mathrm{M}=8.79, \mathrm{SD}=1.65)$. Males made up $80 \%$ of the ADHD group and 54\% of the non-ADHD control group. The sample of children with ADHD utilized in Study 2 is comprised of a subset of the sample from Study 1 ( $n=70)$ who completed the GNG task; however, as noted above, this group also contains a small number of additional 6-year-old children $(n=10)$. The sample of non-ADHD control subjects in Study $2(n=79)$ comprises the same control subjects from Study 1, with the addition of 9 children also due to the widened age range. Among the 
ADHD group, $73 \%$ were diagnosed with combined presentation, $14 \%$ were diagnosed as the predominantly inattentive presentation, and $13 \%$ were diagnosed as the predominantly hyperactive/impulsive presentation (DSM-5; American Psychiatric Association, 2013). Hispanic or Latino children made up a large proportion of the sample (89\% of controls, $91 \%$ of ADHD). Additionally, approximately $14 \%$ of participants (18\% of controls, $10 \%$ of ADHD) were identified by a parent as a racial minority (10.4\% African America, 2.4\% Asian, and 1\% American Indian). Additional demographic and diagnostic details for the Study 2 sample are provided in Table 6. The local Institutional Review Board approved the study and all procedures conformed to the Ethical Principles of Psychologists and Code of Conduct (American Psychological Association, 2002). Parents provided written informed consent and children provided written informed assent.

\section{Procedures}

Recruitment and Exclusion Criteria. See Study 1 Methods; recruitment for the current and exclusion criteria for the current study are identical to those used in Study 1.

Exclusion Criteria. Exclusion criteria for Study 2 are identical to those from Study 1, with two exceptions. First, children as young as 6 years old were not excluded (see Summary and Participants sections, above). Second, children with an abnormally high rate of omitted responses were excluded due to the likelihood that they were noncompliant (see GNG procedures, below, for details).

Diagnostic Procedures and Measures of Psychopathology. Participants in both groups (ADHD and TD) underwent identical processes for screening, establishing 
eligibility, and diagnostic assignment as was described for Study 1. Please see Study 1 Diagnostic Procedures on p. 14 for details.

Medication Washout and Context. These procedures and the context of data collection are identical to those listed in Study 1 Methods on p. 15. All data used in the current study, for the ADHD sample, were obtained while children were taking placebo.

\section{Measures}

Psychopathology. Measures of clinical symptoms was identical to that used in Study 1. Briefly, parent-reported symptoms of ADHD and oppositional defiant disorder were measured by the DBD-RS. A structured interview with the child's parent, the Diagnostic Interview Schedule for Children for DSM-IV (DISC-IV; Shaffer et al., 2000), was also administered. For the purposes of the current study, all comorbid diagnoses were determined on the basis of parent-reported endorsements on the DISC-IV. See page 14 for additional details.

Go/No-Go Task. Subjects completed a computerized Go/No-Go task (GNG) presented using E-Prime 2.0 (Cservenka, Fair \& Nagel, 2014; Hare Tottenham, Davidson, Glover, \& Casey, 2005). This task presented photographs of neutral-affect female and male faces derived from the NimStim Face Stimulus Set ${ }^{2}$. There is ample

${ }^{2}$ Development of the MacBrain Face Stimulus Set was overseen by Nim Tottenham and supported by the John D. and Catherine T. MacArthur Foundation Research Network on Early Experience and Brain Development. Please contact Nim Tottenham at tott0006@tc.umn.edu for more information concerning the stimulus set. 
evidence that these faces are perceived as emotionally neutral by adult and child subjects (www.macbrain.org/resources.htm). The photographed faces were presented in grayscale and were comprised of four females and two males. Child subjects were instructed to responded to female faces (i.e., "Go-Trials") by pressing a button as fast as possible; however, subjects were instructed to not press the button when male faces were displayed (i.e., "No-Go" trials). Stimuli were presented for $500 \mathrm{~ms}$ and the inter-trial interval varied between 2,000 ms and 12,000 ms $(M=5200 \mathrm{~ms})$. The task consisted of 43 trials with 30 "go" targets $75 \%$ and 13 "no-go" trials, resulting in a total task time of $307.5 \mathrm{sec}$ (i.e., five minutes). Similar to previous studies using GNG tasks (e.g., Epstein et al. 2011; Geurts et al., 2008), response trials with a response time of less than $250 \mathrm{~ms}$ were removed, as they were likely accidental presses (Luce, 1986; Usher \& McClelland, 2001); this resulted in $.04 \%$ of trials being excluded from the data set. In addition, subjects with greater than $80 \%$ of non-response to the 30 "go" trials were excluded from analysis as they were likely non-compliant or the result of equipment malfunction; similar criteria are used in previous studies using GNG tasks (e.g., Epstein et al. 2011; Geurts et al., 2008). This accuracy criterion resulted in 6 controls and 2 ADHD subjects (4.8\% of total cases) eliminated from the original dataset.

Physiological Recording and RSA. The methods and equipment for physiological recording and calculation of RSA were identical to that described for Study 1 (pgs. 1617). Specific to Study 2, RSA was derived in 30 second epochs across rest, neutral period, and GNG task. The initial 30 second epoch of these tasks was utilized based on the consideration and reasoning that: 1) this epoch was less prone to the effects of boredom (i.e., still somewhat novel), avoiding additional confounding self-regulatory 
demands; 2) the initial 30s epochs of rest, neutral, and GNG tasks were also utilized in Study 1. Maintaining consistency in methodology will aid in comparing findings across the studies and respective cognitive measures.

Physiological reactivity to task demands were calculated by subtracting average RSA during the first 30sec epoch of the neutral period task from average RSA during the first $30 \mathrm{sec}$ epoch of the GNG task (described below).

$$
\mathrm{RSA}[\mathrm{GNG}]-\mathrm{RSA}[\text { Neutral period] }=\Delta \mathrm{RSA}[\text { reactivity }]
$$

Resting Baseline and Neutral Physiological Period. These procedures are identical to that described in Study 1 (see p. 18 for details). Regarding the neutral period, similar to the procedure described for Study 1, children completed an identical neutral pictures task before the GNG task. The neutral period lasted approximately two minutes and included viewing 10 pictures from the neutral set of the International Affective Picture System (Lang, Bradley \& Cuthbert, 1997) on a computer screen.

\section{Analytic Plan}

Group differences (i.e., ADHD vs. control) in Go/No-Go performance metrics and RSA (i.e., both at rest and reactivity to the task) were examined using analysis of covariance (ANCOVA). The RSA variables included resting RSA, neutral-period RSA, and RSA reactivity during the Go/No-Go task (i.e., RSA change scores calculated as task RSA minus neutral RSA). Go/No-Go performance was indexed by Errors of Commission, Errors of Omission, total errors, and mean reaction time to "Go" trials (MRT). Additionally, linear regression models were used to examine continuous associations among Go/No-Go and RSA variables (as predictors in separate models) and 
ADHD symptom severity (as the outcome in each model). Ancillary analyses will also examine associations with DSM inattentive or hyperactive/impulsive symptom domains as outcomes as well. Finally, regression-based moderation analyses were used to examine the interaction of RSA reactivity and Go/No-Go performance in predicting ADHD (as both a diagnostic category and continuous dimension of symptom severity, in separate models) using the PROCESS macro (Hayes, 2013). Variables were mean-centered prior to moderation analyses. The PROCESS macro performs both linear- and logisticregression-based moderation analyses, probes interaction effects, and provides multiple indices of conditional effects. Pick-a-point effects are given for logistic moderation models, while Johnson-Neyman conditional effects are presented for linear regression moderation models (Bauer \& Curran, 2005; Hayes, 2013). Little's test of missingness examined RSA variables (at rest and on-task), GNG performance variables (total errors), ADHD diagnostic status, gender, and age. Missing data among these variables ranged from $0 \%$ (child gender) to $8.2 \%$ (missing GNG task data) but Little's test indicted no evidence of non-random missingness $\left(\chi^{2}=8.90, p>.35\right)$. Missing data was addressed through list-wise deletion for all models.

\section{Study 2 Results}

\section{Distribution and Power Analysis}

Using G*Power (Faul, Buchner, Lang, 2009), post-hoc power analysis for ANCOVA with two levels indicated power of .77 to detect a medium effect of partial- $\eta^{2}=$ .08 . with a sample of $\mathrm{N}=149$, when covariates and interaction were accounted for. Therefore, the current between-subject analyses are slightly underpowered, as there is a $77 \%$ probability that Type II error will be avoided, and that actual between-subject 
effects will reach statistical significance. For linear multiple regression analyses, power analysis indicated power of .95 to detect a small effect $\left(\mathrm{R}^{2}=.10\right)$ for a sample size of 149 . Post-hoc power analysis for logistic regression indicated power of .92 to detect a medium effect size $($ Odd Ratio $=1.75)$ given a sample of $\mathrm{N}=140$. These proposed effects sizes are consistent with those found in previous research (Ward et al., 2015).

\section{Sample Characteristics}

Table 6 provides demographic and clinical description of both groups. Compared to children in the control group, children with ADHD were more likely to be male $\left(\chi^{2}=\right.$ $10.64, p<.05$ ), which is typical among youth diagnosed with ADHD (APA, 2013). No significant between-group differences were observed in terms of age, estimated full scale IQ, or proportion of racial minority or Hispanic/Latino (all $p>.10$ ).

As expected regarding clinical characteristics, children with ADHD had more symptoms of oppositional defiant disorder (ODD) and conduct disorder (both $p<.001$ ), were more likely to have a diagnosis of $\mathrm{ODD}\left(\chi^{2}=30.48, p<.001\right)$, but not $\mathrm{CD}\left(\chi^{2}=\right.$ $3.24, p=.07)$. As expected, children in the ADHD group had a history of being prescribed medication for ADHD prior to entering the study, but no subjects in the control group were medicated; $\chi^{2}=76.80, p<.001$. These differences are largely representative of children with ADHD in the general population (APA, 2013). No statistically significant between-group differences were observed in diagnoses of anxiety or depressive disorders (all $p>.17$ ). Correlations of the primary variables of interest, demographic, and clinical characteristics are displayed in Table 7a. Child age was significantly correlated with Errors of Omission $(r=-.18, p<.05)$ and mean reaction 
time $(r=-.37, p<.05)$. Age, gender, previous medication status, and ODD symptom count were included as covariates in all models given associations with either the independent variables or outcomes of interest.

\section{Analyses based on ADHD Diagnosis}

Analyses initially focused on the ADHD syndrome as defined by DSM-5 criteria (American Psychiatric Association, 2013).

Go/No-Go Performance by ADHD Diagnostic Status. ANCOVA was used to examine group differences in Go/No-Go performance via Errors of Omission, Errors of Commission, Total Errors, and mean reaction time (MRT) while controlling for covariates (Table 8a). The effect of diagnostic group on Errors of Omission was not significant, $F(6,126)=1.73, p=.19$, nor was the Group effect on Errors of Commission $F(6,126)=0.00, p=.99$, nor Total Errors, $F(6,126)=2.09, p=.15$. However, there was a significant effect of Group on MRT, $F(6,126)=5.01, p<.05, \eta_{p}{ }^{2}=.04$, such that youth with ADHD had slower MRT $(E M M=641.80, S E=25.55)$ relative to control subjects $(E M M=542.35, S E=26.69)$.

Baseline RSA according to ADHD Diagnosis. At resting baseline, youth with ADHD $(E M M=6.07, S E=0.18)$ exhibited significantly lower resting RSA (i.e., an indication of reduced PNS-based regulation) compared to control youth (EMM=7.14, $S E=0.19), F(5,124)=11.88, p<.01, \eta_{p}{ }^{2}=.09$. Youth with ADHD $(E M M=6.09$, $S E=0.20)$ also exhibited significantly lower RSA during the neutral baseline condition compared to control youth $(E M M=6.85, S E=0.20), F(6,126)=4.90, p<.05, \eta_{p}{ }^{2}=.04$. Between-group RSA effects are shown in Table 8a. 
RSA Reactivity Effects according to ADHD Diagnosis. RSA reactivity was indexed by subtracting the RSA values during the neutral baseline condition from RSA values during the Go/No-Go task. ANCOVA revealed no significant between-groups difference in RSA reactivity during the Go/No-Go task, $F(6,126)=1.99, p=.16$ (see Table 8a).

Test of Moderation Effects. To test the hypothesis that the association between ADHD diagnosis and GNG performance would be moderated by RSA reactivity, moderation analyses were conducted using a logistic-regression-based approach (Hayes, 2013). When predicting diagnostic group status (i.e., ADHD vs typically developing), the interaction of total number of GNG errors and RSA reactivity was not statistically significant $(z=0.73, b=0.08, p=.47)$. Follow up analyses were conducted to examine

whether RSA reactivity moderated the relationship between other indices of performance and ADHD diagnosis; however, neither Errors of Omission $(p>.61)$, Errors of Commission $(p>.78)$, nor MRT $(p>.65)$ significantly interacted with RSA reactivity to predict ADHD diagnosis.

\section{Analyses Based on Continuous/Dimensional ADHD Measures}

Multiple regression analyses examined the association between GNG performance (i.e., the same indices used to compare diagnostic groups) as the predictor variable and overall ADHD severity as the outcome variable, which was measured using the mean of parents' ratings (i.e., raw scores) for ADHD symptoms on the DBD-RS (Pelham et al., 1992). To investigate symptom type specificity, mean raw scores for inattentive and hyperactive/impulsive items (also from the DBD-RS) were used to index inattentive and hyperactive/impulsive symptom severity as outcomes in separate models. 
Child age, sex, ODD symptoms, and medication status were treated as covariates in all models described below.

Go/No-Go and Continuous Measures of ADHD. In the model with mean overall ADHD symptom rating as the dependent variable, Errors of Omission were not significantly associated with total ADHD symptom severity rating, $\beta=0.03, t(125)=0.46$, $p=.64$ (Table 9a). However, Errors of Commission were a significant positive predictor of total ADHD symptom severity rating, $\beta=0.12, t(125)=2.01, p<.05$. Additionally, slower mean RT predicted more severe total ADHD symptom ratings $\beta=0.14, t(125)=$ 2.24, $p<.05$. The association of GNG Total Errors (i.e., the sum Errors of Omission and Commission) and ADHD symptom ratings was not significant, $p=.06$.

To investigate whether GNG performance was uniquely associated with individual DSM-5 symptom dimensions, follow up analyses repeated these models using inattentive, and separately, hyperactive/impulsive symptoms as the outcome while controlling for the counterpart dimension and relevant covariates. Errors of Omission were not significantly associated with inattentive symptom rating, $p>.67$ (Table 9b), nor were mean RT ( $p>$ $.21)$, nor Total Errors $(p>.13)$. However, Errors of Commission was a significant positive predictor of inattentive symptom rating, $\beta=0.14, t(125)=2.98, p<.01$. When predicting hyperactive/impulsive symptom ratings, none of the GNG performance indices were significant predictors (all $p>.13$; see Table 9c).

At-Rest RSA and Continuous Measures of ADHD. This sample constitutes a subsample of that examined in Study 1; in addition, the neutral period physiological data are unique to the period immediately prior to the GNG task. Therefore, at-rest and neutral 
period RSA were examined in separate models predicting parent-rated ADHD severity. Results of regression analyses are summarized in Table 9a. The model with overall $\mathrm{ADHD}$ rating as the outcome variable and resting baseline RSA and all covariates as predictors was significant, $F(5,123)=41.17, p<.001, R^{2}=.63$. Specifically, RSA at resting baseline was significant and negatively associated with overall ADHD symptom rating, $\beta=-.21, t(123)=-3.76, p<.001, s r^{2}=-.21$. In a separate model, neutral period RSA was a non-significant predictor of overall ADHD rating, $p>.41$. The findings indicate that lower resting RSA predicts higher-rated ADHD severity, while RSA during a neutral period between tasks was not significantly associated with ADHD symptom severity. Children with higher rated ADHD symptom severity appear to exhibit reduced PNS-regulation over resting heart rate compared to controls.

To examine the specificity of significant findings with respect to DSM-5 ADHD symptom dimensions, multiple regression analyses replicated these analyses with resting RSA as the predictor of interest, and in separate models, inattentive or hyperactive/impulsive symptom ratings as the outcome (see Tables $9 \mathrm{~b}$ and 9c). Resting RSA was a significant negative predictor of inattention, $\beta=-0.12, t(122)=-2.54, p<.05$, $s r^{2}=-.12$, but not hyperactivity/impulsivity $(\beta=-0.01, t(122)=-0.30, p=.77)$. Neutral period RSA was not a significant predictor of inattention symptom severity $(\beta=-0.06$, $t(122)=-1.25, p=.22)$ nor hyperactive symptom severity $(\beta=0.03, t(122)=0.66, p=.51)$. Overall findings suggest that, while at rest, inattentive symptom severity is uniquely predictive of reduced PNS-regulation over heart rate. 
Autonomic Reactivity and Continuous Measures of ADHD. Next, multiple regression was used to examine the association between RSA reactivity and ADHD severity when accounting for covariates, but the RSA-change predictor was not significant $(\beta=.01, t(118)=0.12, p=.90)$. When examining potential specificity with respect to ADHD symptom domains (similar to with resting RSA above), RSA reactivity was not a significant predictor of inattentive severity $(\beta=0.04, t(118)=0.80, p=.79)$ nor hyperactive/impulsive severity $(\beta=-0.03, t(118)=-0.74, p=.46)$.

Test of Moderation Effects on Continuous Measures of ADHD. Moderation analyses examined whether the association between GNG performance and ADHD symptom severity was affected by RSA reactivity; a linear regression approach was utilized (Cohen, Cohen, West \& Aiken, 2003; Hayes, 2013). The interaction of RSA reactivity and total GNG errors was not significant in predicting overall ADHD symptom severity, $\mathrm{b}=-0.01, S E=0.02, t(116)=-0.53, p=.59$. Follow-up analyses examining each of the following interactions predicting ADHD symptom severity were also not significant: total Errors of Commission $\mathrm{x}$ RSA reactivity $(p=.51)$, total Errors of Omission x RSA reactivity ( $p=.81)$, and Mean RT x RSA reactivity ( $p=15)$.

Additional moderation analyses examined the GNG errors x RSA interaction effect in separate models containing either inattentive or hyperactive/impulsive symptom ratings as the outcome (and adding the other respective domain as a covariate). Neither the model predicting inattentive symptom ratings $(p=.17)$ nor hyperactive/impulsive symptom ratings $(p=.23)$ was significant. 
Follow-up analyses examined the moderating effect of RSA reactivity among the ADHD subgroup only $(n=70)$. The interaction of GNG Total Errors and RSA-change was not a significant predictor of ADHD severity $(b=0.01, S E=0.03, p=.21)$. The model with hyperactivity/impulsivity severity as the outcome was also not significant $(b=-0.01$, $S E=0.02, p=.39)$. However, when examining the same interaction with ADHD inattention as the outcome, the interaction was significant $b=0.07, S E=0.03, t(54)=$ $2.04, p<.05$. When probing this interaction, conditional effects indicated that during positive RSA-change (from neutral to task), GNG errors significantly predicted inattention severity among ADHD children (Table 10). Specifically, Johnson-Neyman indices show the region of significance occurring for rather intense RSA elevation in response to the task $\left(92^{\text {nd }}\right.$ percentile and above). This indicates that severe inattentive symptoms were associated with greater overall GNG errors among those children who exhibited the greatest elevations in RSA during the GNG task.

\section{Study 2 Discussion}

Weakened response inhibition and vigilant attention are central to prominent etiological theories of ADHD (Barkley, 1997; Douglas, 1999); however, there is evidence that these effects are heterogeneous at the individual level (Nigg et al., 2005; Lambek et al., 2010; Whalstedt, Thorell \& Bohlin, 2009). PNS-based regulation (indexed by RSA) has proven an influential component of behavioral, cognitive, and emotion regulation in both TD (Marcovitch et al., 2010; Pluess et al., 1994) and clinical groups (Crowell et al., 2006; Musser et al., 2011), to the point of moderating well-established links between cognitive components and ADHD (Kahle et al., 2018; Ward et al., 2015). The current study builds on these findings and the findings in Aim 1 by exploring the interactive 
associations between attentional and inhibitory control, PNS activity, and ADHD (both at the dichotomous level as a diagnosis and at the continuous level by examining symptom severity).

ANCOVA revealed no significant ADHD between-group differences with respect to the number of Errors of Omission or Errors of Commission during the GNG task. However, children with ADHD responded to "Go" targets more slowly on average than their typically developing peers (Table 8a). Additionally, when examined continuously, Errors of Commission were positively associated with parent-rated ADHD symptom severity, and MRT was positively associated with ADHD symptom severity (Table 9a). The latter effect of MRT indicated that longer RT was related to more severe ADHD symptom ratings. Errors of Omission were not associated with ADHD symptom severity, counter to previous research (Borger et al., 1999; Epstein, et al., 2003), and failing to support the hypothesis that this feature would be elevated in the ADHD group. Although other studies have reported null between-group effects for Errors of Omission (e.g., Teicher, Ito, Glod \& Barber, 1996), methodological differences may be the most likely factor, particularly given that the effects of MRT and Errors of Commission were significant. For example, the relatively shorter duration of the GNG task (43 discrete trials, rather than 100 or more, which is common; Conners, Epstein, Angold \& Klaric, 2003) may have reduced the sensitivity of the task to the effects of deficient sustained attention. Notably, deficits in sustained or "vigilant" attention are associated with ADHD while shorter-term selective attention is generally considered to be intact (Huang-Pollock \& Nigg, 2003; Huang-Pollock, Nigg \& Halperin, 2006) - by definition, the effects of a sustained attention deficit are more apparent with longer tasks. 
The findings that children with ADHD exhibit slower MRT and more frequent errors of commission replicate findings from previous literature (Booth et al., 2005; Borger et al., 1999; Carte, Nigg \& Hinshaw, 1996; Lijffijt et al., 2005; Metin et al., 2012; Scheres, Oosterlaan \& Sergeant, 2001; Sediman, Biederman, Faroane, Weber \& Ouellete, 1997). Slower MRT among ADHD groups has been proposed as resulting from dysfunction in motor planning, arousal, or effort (Sergeant, 2005), or as a by-product of greater distractibility in the ADHD groups (Barkley, 1997; Leth-Steensen et al., 2000). Similarly, elevated Errors of Commission have been interpreted both as evidence for a primary deficit in response inhibition (Barkley, 1997), or as a secondary outcome stemming from deficient self-regulation (Douglas, 1999; Sergeant, 2000, 2005). When further contemplating these findings, the possibility that slower MRT among ADHD youth may be reflective of over-cautious responding (i.e., due to poor response inhibition) was considered. However, Errors of Commission were not significantly correlated with MRT ( $r=.01$; Table 7a). Therefore, the current findings reinforce the fact that weakened response inhibition in children with ADHD is a robust characteristic which manifests across different samples, using different tasks, contexts, and stimuli (Epstein et al., 2011; Metin et al., 2012), including when differentiating between male and female faces as in the task used here.

Furthermore, when examining each DSM-5 symptom domain separately, Errors of Commission were uniquely associated with inattentive symptom severity (Table 9b), but not with hyperactive/impulsive symptoms (Table 9c). The finding that problems with response inhibition would be uniquely tied to inattention rather than hyperactive/impulsive symptoms seems counter-intuitive. However, these results align 
with the conclusion of a recent meta-analytic review where deficits in executive functioning (which response inhibition is linked to) are more consistently associated with inattentive symptoms (see Willcutt et al., 2012 for a review). In particular, difficulty inhibiting a pre-potent response, whether driven by underlying disinhibition or difficulty adequately regulating attention or arousal, may be more readily observed as ADHD inattentive symptoms than as hyperactivity/impulsivity symptoms. However, heterogeneity among individuals with ADHD with regard to inhibitory deficits continues to impede progress toward verifying specific developmental pathways involving such associations.

Therefore, analyses next turned to examining PNS regulation at rest and reactivity during the GNG task (indexed via RSA). The effect of resting RSA was examined in Study 1, but verified again in Study 2 due to slight differences in samples between the studies. As expected, effects of RSA during the resting baseline and RSA during the neutral period preceding the task were similar (see Table 8a). Notably, the significant difference in PNS activity observed during the neutral period became negligible during the GNG task.

Attempts to clarify how PNS activity may be involved in ADHD impairment and presentation have led to findings that PNS reactivity moderated associations between short-term memory (an aspect of working memory) and ADHD symptoms (see Study 1; also, Ward et al., 2015). To test whether this moderation effect was specific to short-term memory or also true for other executive function domains, the potential moderating role of RSA-change on the association between GNG errors and ADHD symptom severity 
was examined. Findings were less robust and consistent compared to the findings in Study 1. Specifically, RSA-change was not a significant moderator of links between GNG performance and overall ADHD severity. However, follow-up analysis among the ADHD sample indicated a significant interaction: the association between GNG Errors and ADHD inattention severity was moderated by RSA-change (Figure 3). Exploring conditional effects further revealed that, among children with ADHD, children who exhibited a rather extreme increase in PNS influence during the task along with greater rate of GNG errors (both omissions and commissions) had more severe ADHD inattention (see Table 10 and Figure 3).

These results are similar to findings that PNS regulation is related to performance on cognitive tasks and ADHD symptom presentation (i.e., severity of parent-rated symptoms; Beauchaine et al., 2013; Gao, Borlam \& Zang, 2015; Griffiths et al.,2017; Ward et al., 2015). Here specifically, attentional/inhibitory performance was associated with ADHD inattention, but only for a subset of children with abnormal PNS reactivity. A similar overall finding was found with the relationship between working memory problems and ADHD symptomatology in Study 1, but apparently this moderation effect is not confined to that construct. Thus, there is preliminary evidence that atypically intense PNS reactivity partially explains some of the neuropsychological heterogeneity in ADHD, as it appears that a specific profile of intense PNS reactivity combined with weakened prefrontal-based cognitive functions is particularly associated with the highest symptom severity. 
Of course, there are limitations to consider which temper firm conclusions and highlight needed future directions. First, given the results of post-hoc power analysis for this study (see Results), the follow-up analyses conducted within the ADHD-only group were likely under-powered since utilizing this subgroup effectively reduced the sample size by half (see Table 6). In addition, the current sample was recruited from a treatmentseeking population of children (i.e., children who were enrolled in the Summer Treatment Program), which may indicate some caveat regarding generalizability of the novel findings (i.e., the moderation effect of PNS regulation on the response inhibition-ADHD association). Previous research has suggested that clinic-recruited or treatment-seeking samples in mental health research may constitute a more severely affected population (MacLeod et al., 1999). However, the findings regarding weakened response inhibition and response execution among ADHD youth replicate previous findings (Lijffijt et al., 2005; Oosterlaan, Logan, \& Sergeant, 1998).

Another important consideration involves the phenomenon that children with ADHD not only exhibit neuropsychological heterogeneity between individuals, but task performance may be highly variable within individual children themselves. That is, children with ADHD tend to be inconsistent in their performance on commonly used cognitive tasks (i.e., intra-individual variability), as is becoming well-documented in literature examining response time variability (RTV). In fact, RTV has shown to be a robust correlate of ADHD, with studies indicating a stronger effect for RTV than gross performance indices such as task errors (Epstein et al., 2011; Klein et al., 2006). Therefore, examining the potential interactive effects of PNS regulation and reactivity 
with an index of performance variability such as RTV is undertaken in the third and final aim/study. 
CHAPTER IV: STUDY 3.

\section{Introduction to Study 3}

Observations from clinicians and empirical work have suggested for decades that children with ADHD are more inconsistent compared to their non-ADHD peers with regard to engagement and responding to environmental stimuli, particularly in contexts with high demands on attention or working memory (Cohen \& Douglas, 1972; Douglas 1999). As the use of computerized tasks of attention and response inhibition became more prevalent, this inconsistency was quantified via reaction time fluctuations during such tasks. Reaction time variability (RTV) refers to the trial-to-trial fluctuations in reaction time (RT) across the distribution of an individual's responses on a task. A large literature documents that children with ADHD exhibit elevated RTV compared to typically developing peers (Karalunas, Geurts, Konrad, Bender, \& Nigg, 2014; Kofler, et al., 2013; Lijfijjt et al., 2005). Further, a recent meta-analysis by Kofler and colleagues (2013) obtained an effect size of 0.76 , which is similar to or larger than other cognitive differences observed in ADHD (Martinussen et al., 2005; Willcutt et al., 2005).

Various methods are used to characterize RTV, including measures of overall inconsistency (such as standard deviation), as well as more fine-grained distributional properties of individual-level RT data. The classic and most rudimentary means of characterizing RTV is to calculate the standard deviation of reaction times (SDRT) for each individual in a sample. While SDRT represents an informative index of global variability in RT, it does not account for differences in overall mean RT, which may serve as a confound. In fact, SDRT is often highly correlated with mean RT in studies 
involving non-clinical samples ( $r=.92$; Wagenmakers \& Brown), as well as in samples of children with ADHD ( $r=.74$; Nigg, 2001). Additionally, RT distributions are typically characterized by a prominent positive skew, comprised of a set of larger (i.e. slower) RTs (e.g., Luce, 1986; Luce \& Green, 1972), for which calculations of SDRT are biased (Epstein et al., 2011). That is, in such situations, SDRT does not account for the extent of skew nor the magnitude of influence exerted by the outlying values in the right tail (Luce, 1986).

More recently, the ex-Gaussian distributional model has demonstrated utility for providing quantitative measures which characterize an individual's pattern of responses in more detail (Heathcote, Popiel \& Mewhort, 1991; Ratcliff, 1979). The ex-Gaussian distributional model assumes that the data (i.e., in this case, the distribution of an individual's RTs) are comprised of both a normally distributed (Gaussian) component and an exponential component (Figure 4). Modeling RT data using the ex-Gaussian function yields two parameters related to the Gaussian component, Mu and Sigma, and one parameter of the exponential component, Tau. Mu and Sigma quantify the mean and standard deviation, respectively, of the normally distributed component of RT's, while Tau describes the mean magnitude of the right-hand tail (i.e., positive skew). The exGaussian function is fitted at the individual level, and so the obtained metrics reflect properties of intra-individual variability independent of between-subject differences in task performance or RT. When applied to RT data, larger values of Tau indicate a greater proportion of extreme slow responses and, therefore, a greater influence of excessively slow RTs on the distribution. 
In the context of research on increased RTV as a feature of $\mathrm{ADHD}$, findings from ex-Gaussian studies indicate that children with ADHD exhibit significantly larger values of Tau relative to control groups (Epstein et al., 2011; Hervey et al., 2006; Leth-Steensen, Elbaz, \& Douglas 2000e), indicating more frequent abnormally slow responses. These same studies often further show that Mu (i.e., mean of RT among the Gaussian component) for ADHD youth are not significantly different than TD youth (Epstein et al., 2011), or in some cases faster (Hervey et al., 2006; Leth-Steensen et al., 2000). These studies concluded that findings of slower mean RT and increased SDRT among ADHD samples (Lijffijt et al., 2005; Oosterlaan, Logan, \& Sergeant, 1998) are likely due to a subset of abnormally slow responses (i.e., indexed by a larger Tau), and these abnormally slow responses likely reflect lapses in attention (Henriquez-Henriquez et al., 2015; LethSteensen et al., 2000; Vaurio, Simmonds, Mostofsky, 2009) and/or state-regulation deficits (Karalunas et al., 2014; Geurts et al., 2008).

While many theories indicate that RTV is the result of more frequent attentional lapses among children with ADHD, accounts of the hypothesized underlying neurodevelopmental differences responsible for increased RTV vary (Barkley, 1997; Kofler et al., 2013; Russell et al., 2006; Sergeant, 2005; Sonuga-Barke \& Castellanos, 2007). For instance, Russell and colleagues (2006) hypothesize that intra-individual cognitive and behavioral variability related to ADHD stems from delayed or inconsistent synaptic firing due to neurochemical differences in ADHD neurodevelopment. These neuronal differences lead to lapses of attention and deficits in state regulation (i.e., regulation of effort, arousal, and activation in the face of cognitive demands), which in turn cause variable task performance (see also Sergeant, 2005). 
These hypotheses contribute an intriguing conceptual parallel with the recently burgeoning literature that connects irregular PNS regulation (i.e., vagally mediated, respiratory- driven, high frequency heart-rate variability such as RSA) to domains related to attention, inhibition, and self-regulation (Chapman, Woltering \& Lewis, 2010;

Gianaros, van der Veen \& Jennings, 2004; Holzman \& Bridgett, 2017; Marcovitch et al., 2010; Sturge-Apple et al., 2017; Thayer et al., 2009). With regard to the characteristic problems associated with ADHD, PNS regulation is associated with deficient regulation of behavior, cognitive control, and emotion in children with ADHD and/or related externalizing problems (Borger et al., 1999; Conradt et al., 2014; Rash \& AguirreCamacho, 2012; Kahle et al., 2018; Musser et al., 2011). Specific to observed cognitive heterogeneity among children with ADHD, reactivity of the PNS appears to moderate associations between weakened performance on cognitive tasks and ADHD symptoms. Study 1 showed that PNS reactivity moderates the relationship between working memory and ADHD symptom severity, replicating a previous study (Ward et al., 2015). In Study 2, PNS reactivity moderated the association between Go/No-Go task performance and ADHD inattentive symptoms (independent of hyperactivity), but only when errors of omission and errors of commission were summed (i.e., indexing problems of response inhibition as well as attention, combined). Therefore, PNS reactivity appears to account for some aspects of cognitive heterogeneity that are frequently observed in ADHD, particularly as it relates to working memory, attention, and inhibition (Nigg et al., 2005; Sonuga-Barke, Bitsakou \& Thompson, 2010; Whalstedt et al., 2009).

However, there is a relative dearth of studies examining PNS regulation as it relates to RTV in children with ADHD. This represents a gap in the current literature 
given that effect sizes for RTV differences in children with ADHD are often larger than other frequently used constructs such as attention or response inhibition (Kofler et al., 2013; Karalunas et al., 2014; Martinussen et al., 2005; Willcutt et al., 2005).

The current study evaluated RTV in a group of children diagnosed with ADHD and a group of TD children (i.e., the same sample utilized in Study 2). Distributional properties of RT data were indexed by SDRT (i.e., overall dispersion of RT's), as well as the ex-Gaussian indices of Mu, Sigma, and Tau. It was hypothesized that 1) SDRT will be higher among the ADHD group relative to the TD group, 2) Tau will be greater in the ADHD group compared to the TD group, indicating a greater proportion of abnormally slow RTs, 3) a significant group difference will not be observed for Sigma, indicating that differences in overall variability are not accounted for by differences in the Gaussian portion of the RT distribution, 4) Tau (but not Sigma) will interact with RSA-change to predict ADHD diagnosis and/or ADHD symptom severity.

Similar to Studies 1 and 2, statistical analyses included separate between-subjects comparisons of SDRT, Mu, Sigma, and Tau based on diagnostic group (ADHD vs. TD). In addition, continuous associations between these distributional properties and ADHD symptom severity are tested in separate linear regression models. Finally, moderation analyses examined the interaction of RTV indices (SDRT, Sigma, and Tau) with PNS reactivity (i.e., indexed by RSA-change) in predicting ADHD, both as a diagnostic category and as a continuous outcome (i.e., ADHD symptom severity). 


\section{Study 3 Methods}

\section{Summary}

Study 3 aims to evaluate whether differences in RTV between youth with ADHD and TD youth is moderated by abnormalities in PNS reactivity. RTV was derived from RT data on the same GNG task used in Study 2. Therefore, subject recruitment procedures, measures of psychopathology, and sample characteristics were identical. Herein, RTV is examined both between-groups (i.e., ADHD vs TD) and as it relates to ADHD symptom severity. Finally, moderation analyses investigate interactions between RTV and PNS reactivity (indexed through RSA change from neutral period to task period) in predicting ADHD (both as a diagnosis and in terms of symptom severity).

\section{Participants}

Study 3 utilized the same sample described in Study 2. Details regarding recruitment of participants, identification of diagnoses, and basic descriptive, demographic and clinical data is available in Study 2, pps. 36 - 38 as well as in Table 6. In brief, children between ages 6 and 12 years old, both with and without ADHD, participated in the study.

\section{Procedures}

Recruitment and Exclusion Criteria. Recruitment and exclusion criteria for the current study are identical to those described in Studies 1 and 2. Additional criteria specific to the GNG task data that were incorporated for Study 2 were also used for Study 3 and are detailed on pages 38-39. In brief, participants in the ADHD group were recruited from an existing study on ADHD medication and TD youth were recruited from 
the surrounding community. Exclusion criteria included diagnosed autism spectrum disorder, estimated IQ below 75, seizures, heart conditions, mania, or psychosis.

Diagnostic Procedures and Measures of Psychopathology. These procedures and measures are identical to those described in Studies 1 and 2. Please see p. 14 for details regarding clinical assessment and diagnostic procedures. In brief, both groups were screened and identified using gold-standard assessment procedures including parent and teacher ratings scales, structured parent interview, and standardized intellectual and academic achievement testing. Diagnosis and group assignment were based on independent review of the assessment results by two licensed child psychologists.

\section{Medication Washout and Medication-Based Context. Medication washout} procedures and the medication-based context of research activities are detailed in Study 1 on p. 15. In summary, the larger study from which participants were recruited utilized a double-masked, crossover design in which children were given placebo for either the first or second half of the STP (3 weeks each condition) after an initial 2-week titration period. All data used in the current study, for the ADHD sample, were obtained after children were "washed out" of stimulant medications (equivalent to 7 half-lives or approximately 48 hours), when they were currently administered placebo, rather than active stimulant medication.

\section{Measures}

Psychopathology. Measures of clinical symptoms were the same as those described in Study 1 and Study 2; please see page 14 for details. Briefly, parent-reported symptoms of ADHD and oppositional defiant disorder were measured by the DBD-RS, a well- 
validated rating scale. A structured interview with the child's parent (DISC-IV; Shaffer et al., 2000) was also administered. Symptoms of ADHD and externalizing disorders were measured with the DBD-RS and comorbid anxiety or mood disorders were based on the DISC-IV.

Go/No-Go Task. Data reflecting RT and RTV are derived from the Go/No-Go (GNG) task described in Study 2. A detailed description of the GNG task is provided in Study 2 on pages 38-39. In brief, children were instructed to respond to target stimuli ("go" trials which constitute $75 \%$ of trials) but ignore non-target stimuli ("no-go" trials), all of which presented for $500 \mathrm{~ms}$. The task consisted of 43 trials in total with a varying inter-stimulus interval $(M=5200 \mathrm{~ms})$. Techniques specific to calculating RTV are described in the Analytic Plan section below.

Psychophysiological Recording and RSA. The methods and equipment for physiological recording (via electrocardiogram and impedance cardiograph) and the method used to derive respiratory sinus arrhythmia (RSA) were identical to that described for Studies 1and 2 on page 38-39. The epoch and length of physiological data collected during the GNG are identical to what is detailed for Study 2 on page 39.

Resting and Neutral Physiological Baselines. The procedures for collecting physiological data at rest and during a neutral period preceding the task are identical to those described in Study 1 on page 18. In summary, prior to the administration of any tasks, physiological data were collected during a two-minute period where the child was instructed to relax, refrain from speaking and to stay still (i.e. resting baseline). In 
addition, in order to eliminate potential carry-over from other tasks, physiological data were collected during a neutral period which preceded each task in the battery.

\section{Analytic Plan}

Overall design of statistical analysis was similar to Study 1 and Study 2. Specifically, ANCOVA was used to examine whether RTV was significantly different between the ADHD and TD group. In addition, given evidence that ADHD may be best described in terms of dimensional symptomatology (Willcutt et al., 2012), multiple regression was used to investigate the relationship between RTV and ADHD symptom severity. Ancillary analyses examined associations with DSM inattentive or hyperactive/impulsive symptom domains as outcomes. Finally, a regression-based approach was used to examine the interaction of RSA reactivity and RTV in predicting ADHD (as both a diagnostic category and continuous dimension of symptom severity, in separate models). Details on this approach and probing conditional effects can be found on p. 19 and p. 41.

Calculating ex-Gaussian Parameters. As described in the Introduction to Study 3, response time (RT) data are often characterized by a distribution with most observations clustered in a relatively normally distributed fashion, with a prominent positive skew present (reflecting a subset of slower responses; see Figure 4). For this reason, the exGaussian distribution - which reflects the sum of a normally distributed component (i.e., a Gaussian component) and an exponential (i.e., positive skew) component -- is often used to model RT variability (RTV; Geurts et al., 2008; Heathcote, Popiel, \& Mewhort, 1991; Hervey et al., 2006; Leth-Steenson, Elbaz, \& Douglas, 2000; Metin et al., 2016; 
Vaurio, Simmonds, \& Mostofsky, 2009). The QMPE program (Heathcote, Brown \& Cousineau, 2004) was used to derive the ex-Gaussian parameters of individual RT distributions. QMPE utilizes quantile maximum probability estimation (Heathcote et al., 2004) which has been demonstrated as superior for modeling ex-Gaussian distributions of response times compared to similar methods, including continuous maximum likelihood estimation (Heathcote, Brown \& Mewhort, 2002; Heathcote et al., 2004). Three descriptive parameters are obtained: $\mathrm{Mu}(\mu)$ and Sigma $(\sigma)$, which represent the mean and standard deviation, respectively, of the normal portion of the distribution of response times; as well as Tau $(\tau)$, which describes the exponential component (i.e., the magnitude of positive skew). As reviewed previously, the extent to which RTV is characterized by excessively slow RT (as indexed by Tau) -- relative to variability which is clustered around the mean in a relatively Gaussian manner (indexed by Mu and Sigma) - is an indicator of lapsed attention and/or state regulation (Karalunas et al., 2014; Metin et al., 2016; Vaurio, Simmonds, \& Mostofsky, 2009).

\section{Study 3 Results}

The statistical analyses performed for Study 3 share similar methods, covariates and numbers of independent and dependent variables. Therefore, post-hoc power analyses using G*Power (Faul, Buchner, Lang, 2009) are identical to those presented in Study 2, pages 41-42. In brief, power to detect a medium-sized effect with the ANCOVA for the current sample size and number of covariates was .77, or just below satisfactory. Post-hoc power analysis for the linear regression models reflected power to detect a medium effect of .95 , which is well above satisfactory. 


\section{Sample Characteristics}

Study 3 utilized the same sample and measures as Study 2. Therefore, demographic and clinical description of the sample is provided in Table 6 as well as on p. 42. Overall, the differences between the ADHD and TD group observed for this sample are representative of well-established characteristics of the disorder (APA, 2013), such as more often being male $(p<.01)$, greater prevalence of $\mathrm{ODD}(p<.001)$ and $\mathrm{CD}(p<.01$; there were no CD symptoms or diagnoses among the TD group), and a greater likelihood of being prescribed medication for ADHD prior to the study $(p<.001)$. Within the ADHD group, children prescribed stimulant prescription prior to the study demonstrated faster mean reaction time than those with ADHD who had not been prescribed such medication prior to the study $(p<.05)$. Across the entire sample, male subjects had marginally faster $\mathrm{Mu}(p=.05)$. In addition, child age was significantly correlated with SDRT $(r=-.35)$, Sigma $(r=-.17)$, and Tau $(r=-.30)$. That is, older children were less variable in their responding (i.e., SDRT and Sigma), and had fewer abnormally slow responses (i.e., Tau), consistent with global developmental trends (e.g., Greenberg \& Waldmant, 1993). In light of these effects, and in keeping the covariates utilized in Studies 1 and 2, child age, sex, ODD symptoms, and medication status were included as covariates in all Study 3 analytic models.

\section{Analyses based on ADHD Diagnosis}

One-way ANCOVA was used to examine group differences in SDRT (i.e., an index of overall reaction time variability). In addition, ex-Gaussian functions of $M u$ (i.e., the mean of the Gaussian portion of the RT distribution), Sigma (i.e., the standard 
deviation of an individual's RT's within the Gaussian distribution component), and Tau (i.e., a measure of the extent to which abnormally slow responses contributed to variability) were also compared between diagnostic groups using ANCOVA (see Table 12a).

There was significantly elevated SDRT in the ADHD group (M=128.51, $\mathrm{SE}=12.21)$ compared to the TD group $(\mathrm{M}=78.12, \mathrm{SE}=12.91), F(6,125)=5.56, p<.05$, $\eta_{p}^{2}=.04$, indicating greater overall variability in individual RTs among children with ADHD relative to the TD group (Table 12a). As discussed previously, this finding indicates a global difference in RT fluctuations between groups, but illustrates little in terms of the nature of the difference. When examining measures of the Gaussian component of RT distributions, the effect of diagnostic group on $M u$ was not significant, $F(6,125)=0.25, p>.61$, suggesting that RTs among the Gaussian portion of individuals' RTs were relatively comparable between ADHD and TD groups. Regarding Sigma, the effect was also not significant, $F(6,125)=0.25, p>.61$. The results of these two analyses suggest that, when only the Gaussian portion of the overall distribution is examined, children with ADHD and their TD peers are not clearly differentiated in terms of RT distribution, consistent with previous findings (Leth-Steensen et al., 2000; Vaurio et al., 2009). In contrast, Tau differed significantly according to diagnostic group status, $F(6,125)=5.99, p<.05, \eta^{2} p=.04$, suggesting that the RT distributions of youth with ADHD exhibited greater density in the positively skewed portion compared to TD youth. This overall pattern of findings signifies that the difference in SDRT among individuals in the ADHD group are driven primarily by differences in the magnitude of positive skew 
of RT distributions. That is, children with ADHD tended to produce a greater number of abnormally slow responses relative to TD children.

Test of Moderation Effects. To test the hypothesis that the association between ADHD diagnosis and Tau would be moderated by RSA reactivity, moderation analyses were conducted using a logistic-regression-based approach (Hayes, 2013). When predicting diagnostic group status (i.e., ADHD vs typically developing), the interaction of Tau and RSA reactivity was not statistically significant, $z=0.84, b=0.002, p=.40$.

\section{Analyses Based on Continuous/Dimensional ADHD Measures}

Four regression models examined the association between RTV indices as the predictor variable and ADHD symptom severity as the outcome variable, as measured using the mean parent rating of ADHD symptoms on the DBD-RS (Pelham, Gnagy, Greenslade, \& Milich, 1992). Each predictor (i.e., SDRT, Mu, Sigma, and Tau), together with the covariates specified previously, was examined in a separate model with ADHD symptom severity as the outcome. In addition, ancillary analyses explored whether each RTV index was related to a specific symptom domain (i.e., either inattention or hyperactivity/impulsivity) as measured by the mean parent rating of all symptoms for either domain.

RTV and Continuous Measures of ADHD. In the model with SDRT as the predictor and ADHD symptom rating as the outcome variable, the effect was significant and indicated that greater overall variability in RT (across an individual's entire set of responses) was associated with greater ADHD symptom severity, $\beta=0.13, t(124)=2.08, p$ $<.05$ (Table 13a). When separating the distribution into Gaussian and exponential 
components, $\mathrm{Mu}$ (the mean of RT in the normally distributed portion of RT's) was not significantly related to symptom severity, $\beta=0.05, t(124)=0.90, p=.37$. Additionally, Sigma (the standard deviation of RT's among the Gaussian portion of the distribution) was also not a significant predictor, $\beta=0.05, t(124)=0.86, p=.39$. This finding indicates that, unlike SDRT which reflects variability across the entire RT distribution, variability of RTs within the Gaussian portion is not associated with ADHD symptom severity. The next step was to examine whether the amount of abnormally slow responses was specifically related to ADHD symptomatology. However, the association between Tau and ADHD symptom severity fell short of significance, $\beta=0.12, t(124)=1.96, p=.053$.

Follow up ancillary analyses tested whether the predictors of interest were uniquely related to one of the ADHD symptom domains (i.e., inattentive or hyperactive/impulsive symptom severity). In these analyses, the other respective symptom domain was included as a covariate in order to ascertain unique covariance (e.g., hyperactive symptoms included as a covariate in the model with inattention as the outcome, etc.). However, no significant associations between the distribution characteristics and the individual symptom domains were observed. Specifically, in separate models, neither SDRT ( $p=$ $.09), \mathrm{Mu}(p=.75)$, Sigma $(p=.69)$, nor Tau $(p=.09)$ were significant predictors inattentive symptoms severity; similarly, neither SDRT $(p=.91), \mathrm{Mu}(p=.70)$, Sigma $(p$ $=.27)$, nor Tau $(p=.81)$ were significant predictors hyperactive/impulsive symptoms severity in their respective models. Additional details can be found in Tables $13 \mathrm{~b}$ and $13 \mathrm{c}$.

Test of Moderation Effects on Continuous Measures of ADHD. Moderation analyses examined whether RSA reactivity may moderate associations between RTV 
components and ADHD symptom severity as has been observed with other cognitive correlates of ADHD. As in Study 1 and 2, a linear regression approach was utilized (Cohen, Cohen, West \& Aiken, 2003; Hayes, 2013). Results for all moderation models involving the interaction of SDRT and RSA-change are displayed in Table 14a, and results for all models involving the interaction of Tau and RSA-change are displayed in Table 14b.

The interaction of SDRT and RSA-change fell short of significance in predicting overall ADHD symptom severity, $\mathrm{b}=0.001, S E=0.001, t(115)=1.963, p=.053$. In addition, the interaction of Sigma and RSA-change was not significant, $\mathrm{b}=0.002, S E=$ $0.001, t(115)=1.613, p=.11$, further suggesting that RT variability among the Gaussian portion of RTs was not associated with ADHD symptom severity, even when considering different conditions of RSA reactivity. Finally, the interaction of RSA-change and Tau was not significantly associated with ADHD symptom severity $\mathrm{b}=0.001, S E=<0.001$, $t(115)=1.74, p=.083$.

These results did not support the initial hypotheses that abnormal RSA reactivity would moderate the associations between RTV and ADHD symptom severity. However, previous work - including Studies 1 and 2, and Ward et al., (2015) - indicated that parasympathetic reactivity during cognitive tasks moderated the association between task performance and severity of reported inattentive ADHD symptoms, but not hyperactivity/impulsivity symptoms of ADHD. Therefore, moderation models were repeated with either inattentive symptom severity or hyperactive/impulsive symptom severity as the outcome. Again, the counterpart dimension was included as a covariate in order to confine observed effects of the interaction strictly to the outcome of interest. 
The moderation model with RSA-change as a moderator of the association between SDRT and inattention severity was significant, $\mathrm{b}=0.002, S E=0.001, t(114)=2.66, p<$ .01 (see Table 14a). Conditional effects indicated that subjects with higher RSA-change (i.e., above-average elevation of RSA in response to the task) comprised a group of children for whom SDRT was significantly associated with inattentive symptom severity, but this association was not significant when RSA change values were at mean or belowaverage (see also Figure 5). The Johnson-Neyman region of significance (Johnson \& Neyman, 1936; Bauer \& Curran, 2005) indicated that 27\% of the RSA-change values fell in the elevated range associated with the significant interaction. When examining the interaction of Sigma and RSA-change in predicting ADHD inattention severity, the interaction term was not significant, $\mathrm{b}=0.001, S E=0.001, t(114)=1.18, p=.24$, indicating that RT from the Gaussian portion of RT distribution was (still) not significantly associated with ADHD inattention severity when considering RSA-change conditions. However, when examining the interaction of Tau and RSA-change, the product was a significant predictor of inattention severity, $\mathrm{b}=0.001, S E=<0.001, t(114)=$ 2.03, $p<.05$ (Table 14b). In particular, higher Tau was significantly associated with higher inattention severity ratings when RSA-change was elevated (i.e., above-average RSA elevation in response to the task; see Figure 6).

Follow-up analyses examining the interaction of RTV indices and RSA-change in models with ADHD hyperactive/impulsive symptom severity as the outcome were also conducted. All tested interactions were not significant, including the following interaction terms in separate models: SDRT x RSA-change $(p=.24)$, Sigma x RSA-change $(p=.96)$, and Tau $x$ RSA-change $(p=.48)$. Thus, the degree of RSA-change did not significantly 
alter the association between any of the RTV variables and hyperactive/impulsive symptom severity.

Therefore, these results illustrate that RTV is particularly associated with inattention symptom severity when the RSA response to the GNG task is augmented above average. Importantly, however, when RTV is decomposed according to an exGaussian function, this moderation effect may involve the RTV characterized by Tau (i.e., abnormally slow responses described by exponential distribution) rather than Sigma (i.e., standard deviation of RT's within the normally distributed portion of responses).

\section{Study 3 Discussion}

The current study evaluated associations between RTV and ADHD, as well as the role of PNS regulation as a moderator of these associations. Children with ADHD exhibited greater global RTV (SDRT) compared the TD group. Further, this difference was characterized more specifically by a greater frequency of abnormally slow responses (characterized by Tau) among the ADHD group. However, the normally-distributed portions of individual RT distributions did not reflect significant between-group differences in terms of mean RT $(\mathrm{Mu})$ or standard deviation (Sigma). In linear regression models, higher SDRT was associated with elevated ADHD symptom severity while a positive effect for Tau fell short of significance $(p=.053)$.

These results align with previous studies that implicate RTV as a robust feature of ADHD - linked specifically to a greater proportion of very slow RTs - and manifested across various types of choice-response and attention tasks (Adamo et al., 2014; Epstein et al., 2011; Hervey et al., 2006; Karalunas, Huang-Pollock \& Nigg, 2012; Karalunas et 
al., 2014; Kofler et al., 2013). In particular, these results extend previous findings to a GNG task which involves distinguishing male and female faces (rather than other tasks using text, numbers, or objects), while also bolstering the generalizability of this effect in an ethnically diverse sample of children with ADHD.

Elevated RTV in children with ADHD relative to TD subjects is often interpreted as indicating more frequent lapses of attention (Castellanos et al., 2005; Hervey et al., 2006; Leth-Steensen et al., 2000). However, a primary focus of this line of research is whether RTV may be a common underlying feature of other common cognitive correlates in ADHD - particularly since higher RTV can reasonably be assumed to affect task accuracy and efficiency. RTV has, therefore, been linked to etiological theories of ADHD which emphasize deficiencies of an overarching regulatory process that facilitates attentional engagement, arousal, effort, and/or cognitive inhibitory processes (e.g., Nigg, Goldsmith \& Sachek, 2004; Russel et al., 2006; Sergeant, 2005; Sonuga-Barke \& Castellanos, 2007). More recently, attempts to examine self-regulation in ADHD via objective cardiac-derived measures has become more common in the literature (Musser et al., 2011; Musser \& Nigg, 2017; Rash \& Aguirre-Camacho, 2012) and these indexes of self-regulation have been shown to moderate of the association between ADHD and cognitive correlates (Ward et al., 2015). Therefore, a next step in this study was to conduct moderation analyses, which examined whether the robust association between RTV and ADHD was impacted by PNS-based regulation.

In moderation models with $\mathrm{ADHD}$ symptom severity as the outcome, the interaction of RSA reactivity and SDRT was not significant. In addition, the interaction 
of Tau with RSA reactivity fell short of significance $(p=.08)$. However, when evaluating these effects for specific ADHD symptom domains, it was determined that RSA reactivity moderated the association between SDRT and inattentive symptom severity, and separately, the association between Tau and inattentive symptom severity. For both models, a positive association between the RTV index (i.e., either SDRT or Tau) and inattentive symptom severity was significant only under the condition of an abnormally strong PNS response (i.e., significantly above average elevation in RSA). The correspondence of these findings with the ANCOVA and regression models is somewhat striking. The significant moderation findings involved global RTV (i.e., SDRT) and, when RTV was decomposed into Gaussian and exponential components, RTV linked to the exponential distributional component (i.e., Tau); however, there was no significant moderation effect on the RTV-ADHD association when indexing RTV from the Gaussian portion of the distribution (i.e., Sigma). Thus, if one assumes that Tau is a metric of attentional lapses during the task, it appears that abnormally high PNS reactivity (i.e., excessive RSA elevation) qualified a group for whom more frequent objectively measured attentional lapse (i.e., Tau) did, in fact, map onto higher levels of parent-rated inattentiveness.

Overall, these findings support the conceptualization that abnormal PNS reactivity denotes a sub-optimal preparation to engage with environmental demands and undermines effective self-regulation (Beauchaine \& Thayer, 2015; Porges, 2007; Thayer et al., 2009). With regard to ADHD, a neurobehavioral profile wherein intra-subject RTV is combined with sub-optimal PNS regulation is linked to higher inattentive symptom severity (but not hyperactivity/impulsivity). Although directionality of effects is not 
addressed here, these results encourage further examination of state-regulation factors which may be at play in ADHD psychopathology (e.g., Nigg et al., 2004; Russel et al., 2006; Sergeant, 2005). Recent studies provide behavioral evidence that RTV is reduced among children with ADHD when "state-regulation" is induced by introducing reward conditions and/or modifying event rates during response tasks (Epstein et al., 2011; Kuntsi, Wood, Van der Meere \& Asherson, 2009; Ryan, Martin, Denckla, Mostofsky \& Mahone, 2010). Such contextual modifications are purported to lead to increased arousal and effort, which has positive impacts on performance across response inhibition tasks and RTV among ADHD youth (Andreou et a., 2007; Chee, Logan, Schachar, Lindsay \& Wachsmuth, 1989; Kofler et al., 2013).

There are important limitations to the current study, but which urge future investigations. First, Monte Carlo studies of the analytic method utilized for estimating ex-Gaussian parameters (Quantile Maximum Probability Estimation; QMPE) demonstrates reliable satisfactory performance for samples as small as 40 observations (i.e., 40 trials with responses per individual; Heathcote, Brown \& Mewhort, 2002; Heathcote, Brown \& Cousineau, 2004). However, in the current sample, the mean number of go-trials with a response (i.e., excluding errors of commission) was 24.8 , which is below this number; thus it is important to examine the indices of model convergence and model fit provided by QMPE (Brown, Cousineau \& Heathcote, 2004, p. 2). These indices indicated that the ex-Gaussian parameters of Mu, Sigma, and Tau were "trustworthy" for $\mathrm{n}=102$ cases ( $65 \%)$ and "probably useful" for the remaining $\mathrm{n}=56$ cases (35\%; Brown, Cousineau \& Heathcote, 2004, p. 4). That is, no cases had so few data that the calculated ex-Gaussian parameters would be deemed unreliable. It is also encouraging 
that the current results which utilized ex-Gaussian parameters are markedly consistent with previously published studies - with the exception of slightly smaller effect sizes (see meta-analysis by Kofler et al., 2013). Therefore, this study should be replicated with a task with a higher number of response trials.

Second, while elevated RTV in youth with ADHD is a robust finding observed across various response-choice tasks (Adamo et al., 2014; Karalunas et al., 2014; Kofler et al., 2013), there is evidence of heterogeneity within this effect across individuals with ADHD. For example, Geurts and colleagues (2008) found that youth with ADHD and comorbid autism spectrum disorder exhibited greater RTV compared to ADHD-only youth, although a more recent meta-analysis concluded that RTV effects present in comorbid groups were attributable to ADHD, and not robust in samples of autism only (Karalunas et al., 2014). Henriquez-Henriquez and colleagues (2015) observed that elevated RTV among children with ADHD was particularly prominent for individuals with specific variants of the DRD4 gene. Therefore, the effect of RTV in ADHD is heterogeneous, and possibly not specific to ADHD. These considerations temper conclusions concerning RTV as an indicator of ADHD-specific dysfunction, yet they do not rule out the notion that RTV represents an underlying state dysregulation which transcends psychiatric diagnostic categories, and likely does not represent a unitary underlying process at the cognitive or genotypic level (Karalunas et a., 2014; Kuntsi, 2014). Future work is needed to clarify the extent to which the observed moderation effects of PNS-based regulation are impacted by comorbidity or risk genotype. 
Finally, the current study is cross-sectional and, therefore, conclusions regarding the developmental timing and trajectories of RTV and PNS-based regulation as it relates to ADHD are not possible. However, results from recent studies indicate longitudinal associations between abnormal PNS-based reactivity and increased behavioral or cognitive dysregulation in at-risk populations (Conradt et al. 2014; Kahle, Utendale, Widaman, \& Hastings, 2018), which signifies that longitudinal examinations mirroring the current study are merited. 


\section{CHAPTER V. OVERALL CONCLUSIONS}

This dissertation investigated whether cognitive deficits frequently associated with ADHD would replicate in a treatment-seeking sample and whether the association between these cognitive constructs and ADHD was moderated by PNS activity, a biological component of self-regulation. Across all three studies, severity of ADHD symptoms was associated with poorer performance in measures of working memory, response inhibition and response execution (with the exception of GNG errors of omission, a broad index of vigilance), and attentional lapse. Additionally, while the ADHD group exhibited significantly poorer WM, this effect was not significant in models with covariates. In terms of PNS regulation, the ADHD group was characterized by reduced PNS-based influence over resting heart rate. Most notably, however, moderation analyses consistently revealed that poorer performance across several cognitive constructs was related to greater ADHD symptom severity particularly when task-linked PNS withdrawal was disproportionate relative to the sample mean. With regard to WM, this moderation finding applied to overall ADHD symptom severity (i.e., across both symptom dimensions). When examining response inhibition and RTV as predictors in individual models, PNS withdrawal was a significant moderator in models with inattentive symptom severity as the outcome, but this was not true for hyperactivity/impulsivity.

The significant main effects of cognitive-level measures replicate previous research documenting weakened aspects of executive functioning in children with ADHD (Willcutt et al., 2005) and are broadly consistent with theories regarding the underlying 
cognitive mechanisms related to ADHD (Barkley, 1997; Nigg, 2001; Sergeant, 2005). In addition, the ADHD group was characterized by lower PNS regulation (i.e., RSA during resting baseline) relative to TD group, yielding a medium effect size. These findings replicate the results of a systematic review (Rash \& Aguirre-Camacho, 2012), but run counter to recent meta-analytic findings of null effect for this association (Koenig et al., 2017). However, the analysis by Koenig and colleagues (2017) included only six studies involving children with $\mathrm{ADHD}$, and the authors noted their inability to examine moderators such as age and gender due to a small number of extant studies. While the current results add to a trend of inconsistent findings in this domain of research, examination of the role of PNS regulation in ADHD continues to proliferate the field. Indeed, theoretical models linking autonomic nervous system processes to self-regulation of behavior, cognition, and emotion (Porges, 1995; Thayer \& Lane, 2000; Thayer et al., 2009) have proven a useful framework for investigating potential mechanisms of ADHD across multiple domains of functioning (i.e, behavioral, cognitive, and physiological), including emotion regulation (Graziano \& Garcia, 2017; Leaberry, Rosen, Fogleman, Walerius \& Slaughter, 2018; Musser et al., 2011; Karalunas et al., 2014) and executive functioning (Borger et al., 1999; Utendale et al., 2014; Ward et al., 2015). Porges' (1995) polyvagal theory predicts that PNS-based regulation over heart rate, via the vagus nerve (i.e., the "vagal brake"), facilitates regulated engagement with environmental demands. Applying this model, the reduced resting PNS in the ADHD group may reflect a reduced margin of PNS regulatory capacity, relating higher risk for dysregulated cognition, emotional responding, and social behavior (Beauchaine, 2001; Porges, 1995, 2007) which 
are characteristic of the diagnosis (APA, 2013; Musser \& Nigg, 2017; Nijmeijer et al., 2008; Nigg, Hinshaw \& Huang-Pollock, 2006; Ward et al., 2015).

A particularly notable aspect of the current results is the rather consistent findings of PNS responding as a significant moderator of cognitive function and ADHD symptoms across studies. The overall findings show that abnormally applied PNSregulation during the tasks characterized children with weaker aspects of executive functioning that corresponded with more severe ADHD symptoms. Extant theoretical models describe optimal PNS-based self-regulation as underlying efficient moment-tomoment top-down cognitive control (Thayer et al, 2009). Specifically, Thayer and colleague's (2009) Neurovisceral Integration Theory predicts that, based on the transactional relationships between the autonomic and central nervous systems, the cardiac-derived indices used here also reflect prefrontal cortical functioning (a primary area underlying attention, response inhibition, and working memory; Boes et al., 2008; Bush, Valera \& Seidman, 2005; Collette, Hogge, Salmon, \& Van der Linden, 2006). Empirically, this model has found support where PNS regulation has been found to predict performance on cognitive tasks (Chapman, Woltering, Lamb \& Lewis, 2010; Gianaros et al., 2004; Mezzacappa, Kindlon, Saul \& Earls, 1998; Suess et al., 1994). Additionally, behavioral dysregulation (i.e., elevated behavioral problems and/or ADHD symptoms) has long been associated with abnormalities in PNS response (Beauchaine, Katkin, Strassberg, \& Snarr, 2001; Conradt et al., 2014; Patriquin, Lorenzi, Scarpa, Calkins \& Bell, 2015; Utendale et al., 2014). Therefore, the current findings replicate previous results involving short-term working memory (i.e., Ward et al., 2015) and also extend these findings by suggesting that 1) this moderation effect is generalizable to 
response execution and attention, and 2) compromised neurovisceral integration may be one manifestation of the heterogeneous cognitive and self-regulation mechanisms underlying ADHD.

With regard to developmental models of ADHD specifically, this profile of atypical regulation interacting with executive deficit may map onto "multiple pathway" models of ADHD development (Nigg et al., 2004; Sonuga-Barke, 2005). For example, in Nigg and colleague's (2004) model, primary ADHD-C and ADHD-I were speculated to arise from a pattern of affective dysregulation, and mild executive function or attention deficits in early development. Although affective dysregulation was specified in this model, dysregulated emotional responding in youth with ADHD is shown to coincide with a profile of atypical PNS responding relative to TD youth, such as that observed across the current studies (see: McQuade \& Breaux, 2017; Musser \& Nigg, 2017).

\section{Limitations and Future Directions}

As mentioned in each discussion section, longitudinal work is needed to elucidate how atypical PNS regulation is manifested in the development of ADHD. Development of executive function and related constructs throughout childhood is fairly well characterized (Willoughby, Wirth, \& Blair, 2012; Zelazo \& Carlson, 2012), and the development of PNS-based regulation across childhood has been extensively studied over the past two decades (Bornstein \& Suess, 2000; Calkins \& Keene, 2004; El-Sheikh, 2005; Field \& Diego, 2008). However, studies of the developmental processes regarding these domains as they relate to the etiology and course of ADHD (and psychopathology broadly) are more recent and not specific to ADHD (Beauchaine \& Gazke-Kopp, 2012; 
Beauchaine et al., 2013). Accordingly, it remains unclear whether PNS dysregulation represents a direct contribution toward developing ADHD (i.e. perhaps via deficits in emotion or cognitive regulation) or whether PNS dysregulation emerges concurrent with ADHD symptoms as a result of other etiological factors.

Relatedly, recent empirical findings have shed new light on the development of executive functioning in early childhood. While executive functioning is highly heritable (Kuntsi et al., 2006), a component of this heritability involves socialization processes within parent-child dyads which directly - and indirectly via epigenetic processes - shape self-regulation across early development (i.e., including several aspects of executive function; Bridgett, Burt, Edwards, Deater-Deckard, 2015; Deater-Deckard, 2014). Furthermore, PNS regulation may serve as a mediator or moderator of these developmental links between socialization and self-regulation, including development of deficient self-regulation associated with psychopathology (Calkins, Propper \& MillsKoonce, 2013; Obradovic, 2016). With regard to the link between parenting behavior and ADHD, lower levels of parental expressed warmth are linked to a developmental trajectory of more persistent and severe ADHD symptoms across middle childhood (Musser et al., 2016), and the cross-sectional association between excessive PNS reactivity and ADHD has been found to be moderated by positive parent speech during a dyadic task (Musser et al., 2018).

These findings suggest that longitudinal studies of the relations between PNS regulation, executive function dimensions, and psychopathology should incorporate measures of family environment, parenting styles, and broader socio-economic factors in 
order to more comprehensively chart the development of ADHD (Blair \& Raver, 2012; Bridgett et al., 2015).

One limitation of the current studies, and a second future direction, is the need to compliment these studies by examining the other branch of the autonomic nervous system, the sympathetic nervous system (SNS). Although the SNS has been less frequently studied in relation to executive functioning, it is a physiological component of arousal and emotional reactivity (Bernston et al., 1994; Quigley \& Stifter, 2006). For example, measures of SNS responding (e.g., pre-ejection period) are linked to approachoriented behavior such as those involving reward or punishment outcomes (Berntson et al., 1994; Richter \& Gendolla, 2009). In samples diagnosed with ADHD or related externalizing problems, baseline SNS at rest is typically reduced, and SNS reactivity during tasks involving emotion regulation or reward/punishment is often blunted in these groups relative to typically developing control groups (Beauchaine et al., 2013; Crowell et al., 2006; Tenenbaum et al., 2018). These effects may be at work in executive function processes as well; recently, Tenenbaum et al. (2018) measured pre-ejection period during two versions of the GNG task (i.e., one neutral, and one with facial affect stimuli) in the same sample utilized for the current project. Children with ADHD exhibited increases in SNS-based cardiac reactivity to the task, while TD youth exhibited slight reductions, characterized by a moderate effect size $\left(\eta_{\mathrm{p}}{ }^{2}=0.06\right)$. Therefore, including measures of SNS functioning in future studies on ADHD cognitive mechanisms will yield a more comprehensive understanding of underlying physiological regulation in children with ADHD (Bauer, Quas, \& Boyce, 2002; El-Sheikh et al. 2009). 
Another limitation of the overall design across all three studies involves generalizability of the cognitive measures. A primary variable in each study was a cognitive component (i.e., working memory, response inhibition, attention, RTV) measured by way of a computerized test. However, as many have pointed out, such tests may offer high levels of reliability and internal validity but are less reliable in predicting adaptive functioning in real-world contexts which require the respective cognitive ability (Barkley \& Fischer, 2011; Wilson, 1993). That is, they may demonstrate poor ecological validity. Therefore, as it relates to the cognitive features of ADHD, it is possible that the weaknesses in respective cognitive domains found here may not consistently generalize to observable deficits in these areas in the day-to-day lives of youth with ADHD. Rating scale measures of EF-related behaviors have been developed as way of measuring the observable behaviors which theoretically arise from deficits in executive functioning (Gioia, Guy, Isquith \& Kenworthy, 1996; Barkley, 2012), but research has shown weak correspondence between these two modalities (i.e., laboratory testing versus rating scales) which indicates that unique information may be contributed by either approach. Future work should incorporate a more comprehensive battery of testing for working memory, response inhibition, and attentional control (as noted in the previous discussion sections), and rating scale measures of executive function are a likely to be a valuable compliment (Toplak, West \& Stanovich, 2013).

Finally, the aforementioned limitations aside, the current results will require replication. A strength of the overall project was that Study 1 represents a replication and extension of previous work (Ward et al., 2015) and, therefore, Studies 2 and 3 represent an extension of the replication aim and should be replicated. Furthermore, the current 
studies utilized a relatively unique sample relative to most psychopathology research conducted in the United States as the makeup is majority Hispanic with the majority of subjects coming from families with college-educated parents. Therefore, even the most basic level of replication using identical measures may seek to extend these findings to a more ethnically and socioeconomically diverse sample.

Findings from the three studies have indicated that abnormal physiological regulation may explain the heterogeneous relationships between ADHD and aspects of executive functioning. Further study of these relationships may prove fruitful for progress in prevention and intervention studies related to ADHD. For example, children with the profile of dysregulated PNS response and compromised aspects of executive functioning may respond differently to interventions. Recent research examined a sample of young children just prior to beginning the Incredible Years treatment program and examined effects at the end of treatment. The findings showed that children with a profile of reduced PNS regulation at baseline evidenced less improvement at post-treatment follow up (Beauchaine et al., 2013). In addition, a pair of studies examining an adapted version of Parent-Child Interaction Therapy for young children born prematurely revealed effects of PNS regulation on treatment outcomes. Bagner and colleagues (2012) showed that lower resting RSA was associated with greater improvements in disruptive behavior following treatment, while another study found greater use of positive parenting behaviors was associated with enhanced RSA suppression (i.e. a pattern of moderate RSA withdrawal more often associated with non-clinical samples; Graziano Bagner, Sheinkopf, Vohr \& Lester, 2012). Taken together with the results of the current studies, these findings indicate that PNS regulation is associated with psychopathology but also 
malleable through proximal developmental processes, such as parent-child interactions (Calkins, Graziano, Berdan, Keane \& Degnan, 2008; Calkins et al., 2013).

Ideally, this overall project fits together with other relevant research to form the basis for future investigations of these synergistic processes, within a developmental psychopathology framework, to inform more targeted prevention efforts, reveal meaningful mechanisms of treatment response, and lead to novel and effective treatment approaches. 


\section{LIST OF REFERENCES}

Adamo, N., Di Martino, A., Esu, L., Petkova, E., Johnson, K., Kelly, S., ... \& Zuddas, A. (2014). Increased response-time variability across different cognitive tasks in children with ADHD. Journal of Attention Disorders, 18(5), 434-446.

American Psychiatric Association. (1994). Diagnostic and statistical manual of mental disorders fourth edition. Washington, DC: American Psychiatric Association.

American Psychiatric Association. (2013). Diagnostic and Statistical Manual of Mental Disorders (5th ed.). Arlington, VA: American Psychiatric Publishing.

American Psychological Association (2002). Ethical principles of psychologists and code of conduct. American Psychologist, 57, 1060-1073.

Andreou, P., Neale, B. M., Chen, W., Christiansen, H., Gabriels, I., Heise, A., . . Kuntsi, J. (2007). Reaction time performance in ADHD: Improvement under fastincentive condition and familial effects. Psychological Medicine, 37, 1703-1715.

Baddeley A. D. (2007). Working Memory, Thought and Action. Oxford, UK: Oxford Univ. Press

Bagner, D. M., Graziano, P. A., Jaccard, J., Sheinkopf, S. J., Vohr, B. R., \& Lester, B. M. (2012). An initial investigation of baseline respiratory sinus arrhythmia as a moderator of treatment outcome for young children born premature with externalizing behavior problems. Behavior Therapy, 43(3), 652-665.

Barkley, R. A. (1997). Behavioral inhibition, sustained attention, and executive functions: constructing a unifying theory of ADHD. Psychological Bulletin, 121(1), 65.

Barkley, R. A. (2012). Barkley Deficits in Executive Functioning Scale--Children and Adolescents (BDEFS-CA). New York: Guilford Press.

Barkley, R. A., Anastopoulos, A. D., Guevremont, D. C., \& Fletcher, K. E. (1991). Adolescents with ADHD: patterns of behavioral adjustment, academic functioning, and treatment utilization. Journal of the American Academy of Child \& Adolescent Psychiatry, 30(5), 752-761.

Barkley, R. A., \& Fischer, M. (2011). Predicting impairment in major life activities and occupational functioning in hyperactive children as adults: Self-reported executive function (EF) deficits versus EF tests. Developmental Neuropsychology, 36(2), 137-161.

Barkley, R. A., Fischer, M., Smallish, L., \& Fletcher, K. (2004). Young adult follow-up of hyperactive children: antisocial activities and drug use. Journal of Child Psychology and Psychiatry, 45(2), 195-211. 
Bauer, A. M., Quas, J. A., \& Boyce, W. T. (2002). Associations between physiological reactivity and children's behavior: Advantages of a multisystem approach. Journal of Developmental \& Behavioral Pediatrics, 23(2), 102-113.

Bauer, D. J., \& Curran, P. J. (2005). Probing interactions in fixed and multilevel regression: inferential and graphical techniques. Multivariate Behavioral Research, 40, 373-400. doi:10.1207/s15327906mbr4003_5.

Beauchaine, T. P. (2001). Vagal tone, development, and gray's motivational theory: Toward an integrated model of autonomic nervous system functioning in psychopathology. Development and Psychopathology, 13(2), 183-214.

Beauchaine, T. P. (2012). Physiological markers of emotion and behavior dysregulation in externalizing psychopathology. Monographs of the Society for Research in Child Development, 77(2), 79-86.

Beauchaine, T. P., Gatzke-Kopp, L., \& Mead, H. K. (2007). Polyvagal theory and developmental psychopathology: Emotion dysregulation and conduct problems from preschool to adolescence. Biological Psychology, 74, 174-184.

Beauchaine, T. P., \& Gatzke-Kopp, L. M. (2012). Instantiating the multiple levels of analysis perspective in a program of study on externalizing behavior. Development and Psychopathology, 24(3), 1003-1018.

Beauchaine, T. P., Gatzke-Kopp, L., Neuhaus, E., Chipman, J., Reid, M. J., \& WebsterStratton, C. (2013). Sympathetic-and parasympathetic-linked cardiac function and prediction of externalizing behavior, emotion regulation, and prosocial behavior among preschoolers treated for ADHD. Journal of Consulting and Clinical Psychology, 81(3), 481.

Beauchaine, T. P., Hinshaw, S. P., \& Pang, K. L. (2010). Comorbidity of attentiondeficit/hyperactivity disorder and early-onset conduct disorder: Biological, environmental, and developmental mechanisms. Clinical Psychology: Science and Practice, 17(4), 327-336.

Beauchaine, T. P., Katkin, E. S., Strassberg, Z., \& Snarr, J. (2001). Disinhibitory psychopathology in male adolescents: Discriminating conduct disorder from attention-deficit/hyperactivity disorder through concurrent assessment of multiple autonomic states. Journal of Abnormal Psychology, 110(4), 610.

Beauchaine, T. P., \& Thayer, J. F. (2015). Heart rate variability as a transdiagnostic biomarker of psychopathology. International Journal of Psychophysiology, 98(2), 338-350. 
Bedard, A. C., Nichols, S., Barbosa, J. A., Schachar, R., Logan, G. D., \& Tannock, R. (2002). The development of selective inhibitory control across the life span. Developmental Neuropsychology, 21(1), 93-111.

Berntson, G. G., Bigger, J. T., Eckberg, D. L., Grossman, P., Kaufmann, P. G., Malik, M., ... van, d. M. (1997). Heart rate variability: Origins, methods, and interpretive caveats. Psychophysiology, 34(6), 623-648.

Berntson, G. G., Cacioppo, J. T., \& Quigley, K. S. (1993). Cardiac psychophysiology and autonomic space in humans: empirical perspectives and conceptual implications. Psychological Bulletin, 114(2), 296-322.

Berntson, G. G., Cacioppo, J. T., Binkley, P. F., Uchino, B. N., Quigley, K. S., \& Fieldstone, A. (1994). Autonomic cardiac control. III. Psychological stress and cardiac response in autonomic space as revealed by pharmacological blockades. Psychophysiology, 31(6), 599-608.

Biederman, J., Petty, C. R., Clarke, A., Lomedico, A., \& Faraone, S. V. (2011). Predictors of persistent ADHD: an 11-year follow-up study. Journal of Psychiatric Research, 45(2), 150-155.

Blair, C., \& Raver, C. C. (2012). Child development in the context of adversity: experiential canalization of brain and behavior. American Psychologist, 67(4), 309.

Boes, A.D., Bechara, A., Tranel, D., Anderson, S.W., Richman, L., Nopoulos, P. (2008). Right ventromedial prefrontal cortex: a neuroanatomical correlate of impulse control in boys. Soc. Cogn. Affect. Neurosci. 4, 1-9.

Booth, J. R., Burman, D. D., Meyer, J. R., Lei, Z., Trommer, B. L., Davenport, N. D., ... \& Marsel Mesulam, M. (2005). Larger deficits in brain networks for response inhibition than for visual selective attention in attention deficit hyperactivity disorder (ADHD). Journal of Child Psychology and Psychiatry, 46(1), 94-111.

Börger, N., van Der Meere, J., Ronner, A., Alberts, E., Geuze, R., \& Bogte, H. (1999). Heart rate variability and sustained attention in ADHD children. Journal of Abnormal Child Psychology, 27(1), 25-33.

Börger, N., \& van der Meere, J. (2000). Motor control and state regulation in children with ADHD: a cardiac response study. Biological Psychology, 51(2-3), 247-267.

Bornstein, M. H., \& Suess, P. E. (2000). Child and mother cardiac vagal tone: Continuity, stability, and concordance across the first 5 years. Developmental Psychology, 36(1), 54. 
Bradley, M. M., \& Lang, P. J. (1994). Measuring emotion: The self-assessment manikin and the semantic differential. Journal of Behavioral Therapy and Experimental Psychiatry, 25, 49-59.

Bridgett, D. J., Burt, N. M., Edwards, E. S., \& Deater-Deckard, K. (2015). Intergenerational transmission of self-regulation: A multidisciplinary review and integrative conceptual framework. Psychological Bulletin, 141(3), 602.

Brown, S., Cousineau, D. \& Heathcote, A. (2004) Technical Manual for QMPE v2.18 [White paper]. Retrieved March 1, 2017 from http://newcl.org/software/qmpe.htm

Bush, G., Valera, E. M., \& Seidman, L. J. (2005). Functional neuroimaging of attentiondeficit/hyperactivity disorder: a review and suggested future directions. Biological Psychiatry, 57(11), 1273-1284.

Cacioppo, J. T., Berntson, G. G., Binkley, P. F., Quigley, K. S., Uchino, B. N., \& Fieldstone, A. (1994). Autonomic cardiac control. II. Noninvasive indices and basal response as revealed by autonomic blockades. Psychophysiology, 31(6), 586-598.

Cacioppo, J. T., Uchino, B. N., \& Berntson, G. G. (1994). Individual differences in the autonomic origins of heart rate reactivity: the psychometrics of respiratory sinus arrhythmia and preejection period. Psychophysiology, 31, 412-419.

Calkins, S. D., Graziano, P. A., Berdan, L. E., Keane, S. P., \& Degnan, K. A. (2008). Predicting cardiac vagal regulation in early childhood from maternal-child relationship quality during toddlerhood. Developmental Psychobiology: The Journal of the International Society for Developmental Psychobiology, 50(8), 751-766.

Calkins, S. D., \& Keane, S. P. (2004). Cardiac vagal regulation across the preschool period: Stability, continuity, and implications for childhood adjustment. Developmental Psychobiology: The Journal of the International Society for Developmental Psychobiology, 45(3), 101-112.

Calkins, S. D., Propper, C., \& Mills-Koonce, W. R. (2013). A biopsychosocial perspective on parenting and developmental psychopathology. Development and Psychopathology, 25(4pt2), 1399-1414.

Calkins, S. D., Fox, N. A., \& Marshall, T. R. (1996). Behavioral and physiological antecedents of inhibited and uninhibited behavior. Child Development, 67(2), 523-540.

Carte, E. T., Nigg, J. T., \& Hinshaw, S. P. (1996). Neuropsychological functioning, motor speed, and language processing in boys with and without ADHD. Journal of Abnormal Child Psychology, 24(4), 481-498. 
Casey, B. J., Castellanos, F. X., Giedd, J. N., Marsh, W. L., Hamburger, S. D., Schubert, A. B., ... \& Rapoport, J. L. (1997). Implication of right frontostriatal circuitry in response inhibition and attention-deficit/hyperactivity disorder. Journal of the American Academy of Child \& Adolescent Psychiatry, 36(3), 374-383.

Castellanos, F. X., Sonuga-Barke, E. J., Scheres, A., Di Martino, A., Hyde, C., \& Walters, J. R. (2005). Varieties of attention-deficit/hyperactivity disorder-related intra-individual variability. Biological Psychiatry, 57(11), 1416-1423.

Castellanos, F. X., Sonuga-Barke, E. J., Milham, M. P., \& Tannock, R. (2006). Characterizing cognition in ADHD: beyond executive dysfunction. Trends in Cognitive Sciences, 10(3), 117-123.

Castellanos, F. X., \& Tannock, R. (2002). Neuroscience of attention-deficit/hyperactivity disorder: the search for endophenotypes. Nature Reviews Neuroscience, 3(8), 617.

Chapman, H. A., Woltering, S., Lamm, C., \& Lewis, M. D. (2010). Hearts and minds: Coordination of neurocognitive and cardiovascular regulation in children and adolescents. Biological Psychology, 84(2), 296-303.

Chee, P., Logan, G., Schachar, R., Lindsay, P., \& Wachsmuth, R. (1989). Effects of event rate and display time on sustained attention in hyperactive, normal, and control children. Journal of abnormal Child Psychology, 17(4), 371-391.

Cohen J. (1988). Statistical Power Analysis for the Behavioral Sciences (2nd ed.), Hillsdale, NJ: Erlbaum. pp. 281, 284, 285.

Cohen, J., Cohen, P., West, S. G., \& Aiken, L. S. (2003). Applied Multiple Regression/Correlation Analysis for the Behavioral Sciences. Mahwah, NJ: Lawrence Erlbaum Associates.

Cohen, N. J., \& Douglas, V. I. (1972). Characteristics of the orienting response in hyperactive and normal children. Psychophysiology, 9(2), 238-245.

Collette, F., Hogge, M., Salmon, E., Van der Linden, M. (2006). Exploration of the neural substrates of executive functioning by functional neuroimaging. Neuroscience 139, 209-221.

Conners, C. K., Epstein, J. N., Angold, A., \& Klaric, J. (2003). Continuous performance test performance in a normative epidemiological sample. Journal of Abnormal Child Psychology, 31(5), 555-562.

Conradt, E., Degarmo, D., Fisher, P., Abar, B., Lester, B. M., Lagasse, L. L., ... \& Hammond, J. A. (2014). The contributions of early adverse experiences and trajectories of respiratory sinus arrhythmia on the development of 
neurobehavioral disinhibition among children with prenatal substance exposure. Development and Psychopathology, 26(4pt1), 901-916.

Conway, A. R., Kane, M. J., Bunting, M. F., Hambrick, D. Z., Wilhelm, O., \& Engle, R. W. (2005). Working memory span tasks: A methodological review and user's guide. Psychonomic Bulletin \& Review, 12, 769-786.

Cousineau, D., Brown, S., \& Heathcote, A. (2004). Fitting distributions using maximum likelihood: Methods and packages. Behavior Research Methods, Instruments, \& Computers, 36(4), 742-756.

Crowell, S. E., Beauchaine, T. P., Gatzke-Kopp, L., Sylvers, P., Mead, H., \& ChipmanChacon, J. (2006). Autonomic correlates of attention-deficit/hyperactivity disorder and oppositional defiant disorder in preschool children. Journal of Abnormal Psychology, 115, 174.

Cservenka, A., Fair, D. A., \& Nagel, B. J. (2014). Emotional processing and brain activity in youth at high risk for alcoholism. Alcoholism: Clinical and Experimental Research, 38(7), 1912-1923.

Deater-Deckard, K. (2014). Family matters: Intergenerational and interpersonal processes of executive function and attentive behavior. Current Directions in Psychological Science, 23(3), 230-236.

Dennis, M., Francis, D. J., Cirino, P. T., Schachar, R., Barnes, M. A., \& Fletcher, J. M. (2009). Why IQ is not a covariate in cognitive studies of neurodevelopmental disorders. Journal of the International Neuropsychological Society, 15(3), 331343.

Douglas, V. I. (1999). Cognitive control processes in attention deficit hyperactivity disorder. In: H. C. Quay, \& A. E. Hogan (Eds.), Handbook of Disruptive Behavior Disorders (pp. 105-138). New York: Plenum Press.

Duschek, S., Muckenthaler, M., Werner, N., \& del Paso, G. A. R. (2009). Relationships between features of autonomic cardiovascular control and cognitive performance. Biological Psychology, 81(2), 110-117.

El-Sheikh, M. (2005). Stability of respiratory sinus arrhythmia in children and young adolescents: A longitudinal examination. Developmental Psychobiology: The Journal of the International Society for Developmental Psychobiology, 46(1), 66-74.

El-Sheikh, M., Kouros, C. D., Erath, S., Cummings, E. M., Keller, P., \& Staton, L. (2009). Marital conflict and children's externalizing behavior: Pathways involving interactions between parasympathetic and sympathetic nervous system activity. Monographs of the Society for Research in Child Development, 74(1), 179 . 
Engle, R. W. (2002). Working memory capacity as executive attention. Current Directions in Psychological Science, 11, 19-23.

Engle, R. W., Tuholski, S. W., Laughlin, J. E., \& Conway, A. R. (1999). Working memory, short-term memory, and general fluid intelligence: a latent-variable approach. Journal of Experimental Psychology: General, 128, 309.

Epstein, J. N., Erkanli, A., Conners, C. K., Klaric, J., Costello, J. E., \& Angold, A. (2003). Relations between continuous performance test performance measures and ADHD behaviors. Journal of Abnormal Child Psychology, 31(5), 543-554.

Epstein, J. N., Langberg, J. M., Rosen, P. J., Graham, A., Narad, M. E., Antonini, T. N., ... \& Altaye, M. (2011). Evidence for higher reaction time variability for children with ADHD on a range of cognitive tasks including reward and event rate manipulations. Neuropsychology, 25(4), 427.

Fabiano, G. A., Pelham, Jr, W. E., Waschbusch, D. A., Gnagy, E. M., Lahey, B. B., Chronis, A. M., ... \& Burrows-MacLean, L. (2006). A practical measure of impairment: psychometric properties of the impairment rating scale in samples of children with attention deficit hyperactivity disorder and two school-based samples. Journal of Clinical Child and Adolescent Psychology, 35(3), 369-385.

Fair, D. A., Bathula, D., Nikolas, M. A., \& Nigg, J. T. (2012). Distinct neuropsychological subgroups in typically developing youth inform heterogeneity in children with ADHD. Proceedings of the National Academy of Sciences, 109(17), 6769-6774.

Faul, F., Erdfelder, E., Buchner, A., \& Lang, A.-G. (2009). Statistical power analyses using $\mathrm{G}^{*}$ Power 3.1: Tests for correlation and regression analyses. Behavior Research Methods, 41, 1149-1160.

Field, T., \& Diego, M. (2008). Vagal activity, early growth and emotional development. Infant Behavior and Development, 31(3), 361-373.

Fray, P. J., Robbins, T. W., \& Sahakian, B. J. (1996). Neuropsychiatric applications of CANTAB. International Journal of Geriatric Psychiatry, 11, 329-336.

Froehlich, T. E., Lanphear, B. P., Epstein, J. N., Barbaresi, W. J., Katusic, S. K., \& Kahn, R. S. (2007). Prevalence, recognition, and treatment of attentiondeficit/hyperactivity disorder in a national sample of US children. Archives of Pediatrics \& Adolescent Medicine, 161(9), 857-864.

Gao, Y., Borlam, D., \& Zhang, W. (2015). The association between heart rate reactivity and fluid intelligence in children. Biological Psychology, 107, 69-75.

Geurts, H. M., Grasman, R. P., Verté, S., Oosterlaan, J., Roeyers, H., van Kammen, S. M., \& Sergeant, J. A. (2008). Intra-individual variability in ADHD, autism 
spectrum disorders and Tourette's syndrome. Neuropsychologia, 46(13), 30303041.

Gianaros, P. J., Van der Veen, F. M., \& Jennings, J. R. (2004). Regional cerebral blood flow correlates with heart period and high-frequency heart period variability during working-memory tasks: Implications for the cortical and subcortical regulation of cardiac autonomic activity. Psychophysiology, 41, 521-530.

Gioia, G. A., Guy, S. C., Isquith, P. K., \& Kenworthy, L. (1996). Behavior rating inventory of executive function. Psychological Assessment Resources.

Graziano, P. A., Bagner, D. M., Sheinkopf, S. J., Vohr, B. R., \& Lester, B. M. (2012). Evidence-based intervention for young children born premature: Preliminary evidence for associated changes in physiological regulation. Infant Behavior and Development, 35(3), 417-428.

Graziano, P., \& Derefinko, K. (2013). Cardiac vagal control and children's adaptive functioning: A meta-analysis. Biological Psychology, 94, 22-37.

Graziano, P. A., \& Garcia, A. (2016). Attention-deficit hyperactivity disorder and children's emotion dysregulation: A meta-analysis. Clinical Psychology Review, 46, 106-123.

Greenberg, L. M., \& Waldmant, I. D. (1993). Developmental normative data on the test of variables of attention (TOVA ${ }^{\mathrm{TM}}$ ). Journal of Child Psychology and Psychiatry, 34(6), 1019-1030.

Griffiths, K. R., Quintana, D. S., Hermens, D. F., Spooner, C., Tsang, T. W., Clarke, S., \& Kohn, M. R. (2017). Sustained attention and heart rate variability in children and adolescents with ADHD. Biological Psychology, 124, 11-20.

Gropper, R. J., \& Tannock, R. (2009). A pilot study of working memory and academic achievement in college students with ADHD. Journal of Attention Disorders, 12, 574-581.

Gross, J. J. (1998). Antecedent- and response-focused emotion regulation: Divergent consequences for experience, expression, and physiology. Journal of Personality and Social Psychology, 74(1), 224-237.

Hale, S. (1990). A global developmental trend in cognitive processing speed. Child Development, 61(3), 653-663.

Hare, T. A., Tottenham, N., Galvan, A., Voss, H. U., Glover, G. H., \& Casey, B. J. (2008). Biological substrates of emotional reactivity and regulation in adolescence during an emotional go-nogo task. Biological Psychiatry, 63(10), 927-934. 
Harvey, P. O., Le Bastard, G., Pochon, J. B., Levy, R., Allilaire, J. F., Dubois, B. E. E. A., \& Fossati, P. (2004). Executive functions and updating of the contents of working memory in unipolar depression. Journal of Psychiatric Research, 38(6), 567-576.

Hansen, A. L., Johnsen, B. H., \& Thayer, J. F. (2003). Vagal influence on working memory and attention. International Journal of Psychophysiology, 48(3), 263274.

Hansen, A. L., Johnsen, B. H., Sollers, J. J., Stenvik, K., \& Thayer, J. F. (2004). Heart rate variability and its relation to prefrontal cognitive function: the effects of training and detraining. European Journal of Applied Physiology, 93(3), 263-272.

Hare, T. A., Tottenham, N., Davidson, M. C., Glover, G. H., \& Casey, B. J. (2005). Contributions of amygdala and striatal activity in emotion regulation. Biological Psychiatry, 57(6), 624-632.

Hayes, A. F. (2013). Introduction to mediation, moderation, and conditional process analysis: A regression-based approach. New York, NY: Guilford Press.

Heathcote, A., Brown, S., \& Mewhort, D. J. K. (2002). Quantile maximum likelihood estimation of response time distributions. Psychonomic Bulletin \& Review, 9(2), 394-401.

Heathcote, A., Brown, S., \& Cousineau, D. (2004). QMPE: Estimating Lognormal, Wald, and Weibull RT distributions with a parameter-dependent lower bound. Behavior Research Methods, Instruments, \& Computers, 36(2), 277-290.

Heathcote, A., Popiel, S. J., \& Mewhort, D. J. K. (1991). Analysis of response time distributions: An example using the Stroop task. Psychological Bulletin, 109, 340347.

Henríquez-Henríquez, M. P., Billeke, P., Henríquez, H., Zamorano, F. J., Rothhammer, F., \& Aboitiz, F. (2015). Intra-individual response variability assessed by exGaussian analysis may be a new endophenotype for attention-deficit/hyperactivity disorder. Frontiers in Psychiatry, 5,197.

Hervey, A. S., Epstein, J. N., Curry, J. F., Tonev, S., Eugene Arnold, L., Keith Conners, C., ... \& Hechtman, L. (2006). Reaction time distribution analysis of neuropsychological performance in an ADHD sample. Child Neuropsychology, 12(2), 125-140.

Higgins, J. P., Thompson, S. G., Deeks, J. J., \& Altman, D. G. (2003). Measuring inconsistency in meta-analyses. BMJ: British Medical Journal, 327(7414), 557. 
Hinnant, J. B., \& El-Sheikh, M. (2009). Children's externalizing and internalizing symptoms over time: The role of individual differences in patterns of RSA responding. Journal of Abnormal Child Psychology, 37(8), 1049.

Holzman, J. B., \& Bridgett, D. J. (2017). Heart rate variability indices as bio-markers of top-down self-regulatory mechanisms: a meta-analytic review. Neuroscience \& Biobehavioral Reviews, 74, 233-255.

Huang-Pollock, C. L., \& Nigg, J. T. (2003). Searching for the attention deficit in attention deficit hyperactivity disorder: The case of visuospatial orienting. Clinical Psychology Review, 23(6), 801-830.

Huang-Pollock, C. L., Nigg, J. T., \& Halperin, J. M. (2006). Single dissociation findings of ADHD deficits in vigilance but not anterior or posterior attention systems. Neuropsychology, 20(4), 420.

Ioannidis, J. P. (2005). Why most published research findings are false. PLoS Medicine, 2(8), e124.

Ioannidis, J. P. (2012). Why science is not necessarily self-correcting. Perspectives on Psychological Science, 7(6), 645-654.

Johnson, P. O., \& Neyman, J. (1936). Tests of certain linear hypotheses and their application to some educational problems. Statistical Research Memoirs, 1, 5793.

Kahle, S., Utendale, W. T., Widaman, K. F., \& Hastings, P. D. (2018). Parasympathetic regulation and inhibitory control predict the development of externalizing problems in early childhood. Journal of Abnormal Child Psychology, 46(2), 237249.

Karalunas, S. L., Geurts, H. M., Konrad, K., Bender, S., \& Nigg, J. T. (2014). Annual research review: Reaction time variability in ADHD and autism spectrum disorders: Measurement and mechanisms of a proposed trans-diagnostic phenotype. Journal of Child Psychology and Psychiatry, 55(6), 685-710.

Karalunas, S. L., Huang-Pollock, C. L., \& Nigg, J. T. (2012). Decomposing attentiondeficit/hyperactivity disorder (ADHD)-related effects in response speed and variability. Neuropsychology, 26(6), 684.

Kasper, L. J., Alderson, R. M., \& Hudec, K. L. (2012). Moderators of working memory deficits in children with attention-deficit/hyperactivity disorder (ADHD): a metaanalytic review. Clinical Psychology Review, 32(7), 605-617.

Kessler, R. C., Adler, L., Barkley, R., Biederman, J., Conners, C. K., Demler, O., ... \& Spencer, T. (2006). The prevalence and correlates of adult ADHD in the United 
States: results from the National Comorbidity Survey Replication. American Journal of Psychiatry, 163(4), 716-723.

Klein, C., Wendling, K., Huettner, P., Ruder, H., \& Peper, M. (2006). Intra-subject variability in attention-deficit hyperactivity disorder. Biological Psychiatry, 60(10), 1088-1097.

Koenig, J., Rash, J. A., Kemp, A. H., Buchhorn, R., Thayer, J. F., \& Kaess, M. (2017). Resting state vagal tone in attention deficit (hyperactivity) disorder: A metaanalysis. The World Journal of Biological Psychiatry, 18(4), 256-267.

Kofler, M. J., Rapport, M. D., Sarver, D. E., Raiker, J. S., Orban, S. A., Friedman, L. M., \& Kolomeyer, E. G. (2013). Reaction time variability in ADHD: a meta-analytic review of 319 studies. Clinical Psychology Review, 33(6), 795-811.

Kuntsi, J., Rogers, H., Swinard, G., Borger, N., van der Meere J. A. A. P., Rijsdijk, F., \& Asherson, P. (2006). Reaction time, inhibition, working memory and 'delay aversion' performance: genetic influences and their interpretation. Psychological Medicine, 36(11), 1613-1624.

Kuntsi, J., Wood, A. C., Van Der Meere, J., \& Asherson, P. (2009). Why cognitive performance in ADHD may not reveal true potential: Findings from a large population-based sample. Journal of the International Neuropsychological Society, 15, 570-579.

Lambek, R., Tannock, R., Dalsgaard, S., Trillingsgaard, A., Damm, D., \& Thomsen, P. H. (2010). Validating neuropsychological subtypes of ADHD: how do children with and without an executive function deficit differ?. Journal of Child Psychology and Psychiatry, 51(8), 895-904.

Landrø, N. I., Stiles, T. C., \& Sletvold, H. (2001). Neuropsychological function in nonpsychotic unipolar major depression. Cognitive and Behavioral Neurology, 14(4), 233-240.

Lang, P. J., Bradley, M. M., \& Cuthbert, B. N. (1997). International affective picture system (IAPS): Technical manual and affective ratings. NIMH Center for the Study of Emotion and Attention, 39-58.

Langberg, J. M., Epstein, J. N., Altaye, M., Molina, B. S., Arnold, L. E., \& Vitiello, B. (2008). The transition to middle school is associated with changes in the developmental trajectory of ADHD symptomatology in young adolescents with ADHD. Journal of Clinical Child \& Adolescent Psychology, 37(3), 651-663.

Leaberry, K. D., Rosen, P. J., Fogleman, N. D., Walerius, D. M., \& Slaughter, K. E. (2018). Physiological Emotion Regulation in Children with ADHD with and without 
Comorbid Internalizing Disorders: a Preliminary Study. Journal of Psychopathology and Behavioral Assessment, 1-13.

Lemery-Chalfant, K., Doelger, L., \& Goldsmith, H. H. (2008). Genetic relations between effortful and attentional control and symptoms of psychopathology in middle childhood. Infant and Child Development: An International Journal of Research and Practice, 17(4), 365-385.

Leth-Steensen, C., Elbaz, Z. K., \& Douglas, V. I. (2000). Mean response times, variability, and skew in the responding of ADHD children: a response time distributional approach. Acta Psychologica, 104(2), 167-190.

Lijffijt, M., Kenemans, J. L., Verbaten, M. N., \& van Engeland, H. (2005). A metaanalytic review of stopping performance in attention-deficit/hyperactivity disorder: deficient inhibitory motor control?. Journal of Abnormal Psychology, 114(2), 216.

Luce, R. D. (1986). Response times: Their role in inferring elementary mental organization (No. 8). Oxford University Press on Demand.

Luce, R. D., \& Green, D. M. (1972). A neural timing theory for response times and the psychophysics of intensity. Psychological Review, 79(1), 14.

MacLeod, R. J., McNamee, J. E., Boyle, M. H., Offord, D. R., \& Friedrich, M. (1999). Identification of childhood psychiatric disorder by informant: Comparisons of clinic and community samples. The Canadian Journal of Psychiatry, 44(2), 144150 .

Marcovitch, S., Leigh, J., Calkins, S. D., Leerks, E. M., O'Brien, M., \& Blankson, A. N. (2010). Moderate vagal withdrawal in 3.5-year-old children is associated with optimal performance on executive function tasks. Developmental Psychobiology, 52, 603-608.

Martinussen, R., Hayden, J., Hogg-Johnson, S., \& Tannock, R. (2005). A meta-analysis of working memory impairments in children with attention-Deficit/hyperactivity disorder. Journal of the American Academy of Child and Adolescent Psychiatry, 44, 377.

Maxwell, S. E., Lau, M. Y., \& Howard, G. S. (2015). Is psychology suffering from a replication crisis? What does "failure to replicate" really mean?. American Psychologist, 70(6), 487.

McQuade, J. D., \& Breaux, R. P. (2017). Are elevations in ADHD symptoms associated with physiological reactivity and emotion dysregulation in children?. Journal of Abnormal Child Psychology, 45(6), 1091-1103. 
Metin, B., Roeyers, H., Wiersema, J. R., van der Meere, J., \& Sonuga-Barke, E. (2012). A meta-analytic study of event rate effects on $\mathrm{Go} / \mathrm{No}-\mathrm{Go}$ performance in attention-deficit/hyperactivity disorder. Biological Psychiatry, 72(12), 990-996.

Metin, B., Wiersema, J. R., Verguts, T., Gasthuys, R., van Der Meere, J. J., Roeyers, H., \& Sonuga-Barke, E. (2016). Event rate and reaction time performance in ADHD: Testing predictions from the state regulation deficit hypothesis using an exGaussian model. Child Neuropsychology, 22(1), 99-109.

Mezzacappa, E., Kindlon, D., Saul, J. P., \& Earls, F. (1998). Executive and motivational control of performance task behavior, and autonomic heart-rate regulation in children: physiologic validation of two-factor solution inhibitory control. The Journal of Child Psychology and Psychiatry and Allied Disciplines, 39(4), 525531.

Miller, J. G., Chocol, C., Nuselovici, J. N., Utendale, W. T., Simard, M., \& Hastings, P. D. (2013). Children's dynamic RSA change during anger and its relations with parenting, temperament, and control of aggression. Biological Psychology, 92(2), 417-425.

MindWare (2012). MindWare Heart Rate Variability V. 2.6 system. Gahanna: MindWare Technologies.

Molina, B. S., \& Pelham Jr, W. E. (2003). Childhood predictors of adolescent substance use in a longitudinal study of children with ADHD. Journal of Abnormal Psychology, 112(3), 497.

Morgan, P. L., Staff, J., Hillemeier, M. M., Farkas, G., \& Maczuga, S. (2013). Racial and ethnic disparities in ADHD diagnosis from kindergarten to eighth grade. Pediatrics, peds-2012.

Mullineaux, P. Y., Deater-Deckard, K., Petrill, S. A., Thompson, L. A., \& DeThorne, L. S. (2009). Temperament in middle childhood: A behavioral genetic analysis of fathers' and mothers' reports. Journal of Research in Personality, 43(5), 737-746.

Musser, E. D., Backs, R. W., Schmitt, C. F., Ablow, J. C., Measelle, J. R., \& Nigg, J. T. (2011). Emotion regulation via the autonomic nervous system in children with attention-deficit/hyperactivity disorder (ADHD). Journal of Abnormal Child Psychology, 39(6), 841-852.

Musser, E. D., Galloway-Long, H. S., Frick, P. J., \& Nigg, J. T. (2013). Emotion regulation and heterogeneity in attention-deficit/hyperactivity disorder. Journal of the American Academy of Child \& Adolescent Psychiatry, 52(2), 163-171. 
Musser, E. D., Karalunas, S. L., Dieckmann, N., Peris, T. S., \& Nigg, J. T. (2016). Attention-deficit/hyperactivity disorder developmental trajectories related to parental expressed emotion. Journal of Abnormal Psychology, 125(2), 182.

Musser, E. D., Lugo, Y., Ward, A. R., Tenenbaum, R. B., Morris, S., Brijmohan, N., \& Martinez, J. (2018). Parent Emotion Expression and Autonomic-Linked Emotion Dysregulation in Childhood ADHD. Journal of Psychopathology and Behavioral Assessment, 1-13.

Musser, E. D., \& Nigg, J. T. (2017). Emotion dysregulation across emotion systems in attention deficit/hyperactivity disorder. Journal of Clinical Child \& Adolescent Psychology, 1-13.

Negrao, B. L., Bipath, P., van der Westhuizen, D., \& Viljoen, M. (2011). Autonomic correlates at rest and during evoked attention in children with attentiondeficit/hyperactivity disorder and effects of methylphenidate. Neuropsychobiology, 63(2), 82-91.

Nigg, J. T. (1999). The ADHD response-inhibition deficit as measured by the stop task: Replication with DSM-IV combined type, extension, and qualification. Journal of Abnormal Child Psychology, 27(5), 393-402.

Nigg, J. T. (2001). Is ADHD a disinhibitory disorder?. Psychological Bulletin, 127(5), 571-598.

Nigg, J. T. (2010). Attention-deficit/hyperactivity disorder: Endophenotypes, structure, and etiological pathways. Current Directions in Psychological Science, 19(1), 2429.

Nigg, J. T., \& Casey, B. J. (2005). An integrative theory of attention-deficit/hyperactivity disorder based on the cognitive and affective neurosciences. Development and Psychopathology, 17(3), 785-806.

Nigg, J. T., Goldsmith, H. H., \& Sachek, J. (2004). Temperament and attention deficit hyperactivity disorder: The development of a multiple pathway model. Journal of Clinical Child and Adolescent Psychology, 33(1), 42-53.

Nigg, J. T., Hinshaw, S. P. and Huang-Pollock, C. (2015). Disorders of attention and impulse regulation. In Developmental Psychopathology: Volume Three: Risk, Disorder, and Adaptation (eds D. Cicchetti and D. J. Cohen), 358-403.

Nigg, J. T., Hinshaw, S. P., \& Huang-Pollock, C. (2015). Disorders of attention and impulse regulation. Developmental Psychopathology: Volume Three: Risk, Disorder, and Adaptation, 358-403.

Nigg, J. T., Willcutt, E. G., Doyle, A. E., \& Sonuga-Barke, E. J. S. (2005). Causal heterogeneity in attention-deficit/ hyperactivity disorder: Do we need 
neuropsychologically impaired subtypes? Biological Psychiatry, 57(11), 12241230.

Nijmeijer, J. S., Minderaa, R. B., Buitelaar, J. K., Mulligan, A., Hartman, C. A., \& Hoekstra, P. J. (2008). Attention-deficit/hyperactivity disorder and social dysfunctioning. Clinical Psychology Review, 28(4), 692-708.

Obradović, J. (2016). Physiological responsivity and executive functioning: Implications for adaptation and resilience in early childhood. Child Development Perspectives, 10(1), 65-70.

Obradović, J., \& Boyce, W. T. (2012). Developmental psychophysiology of emotion processes. Monographs of the Society for Research in Child Development, 77(2), 120-128.

Oosterlaan, J., Logan, G. D., \& Sergeant, J. A. (1998). Response inhibition in AD/HD, $\mathrm{CD}$, comorbid $\mathrm{AD} / \mathrm{HD}+\mathrm{CD}$, anxious, and control children: A meta-analysis of studies with the stop task. The Journal of Child Psychology and Psychiatry and Allied Disciplines, 39(3), 411-425.

Pashler, H., \& Wagenmakers, E. J. (2012). Editors' introduction to the special section on replicability in psychological science: A crisis of confidence? Perspectives on Psychological Science, 7(6), 528-530.

Pastor, P. N., \& Reuben, C. A. (2008). Diagnosed attention deficit hyperactivity disorder and learning disability: United States, 2004-2006. Vital and Health Statistics. Series 10, Data from the National Health Survey, (237), 1-14.

Patriquin, M. A., Lorenzi, J., Scarpa, A., Calkins, S. D., \& Bell, M. A. (2015). Broad implications for respiratory sinus arrhythmia development: Associations with childhood symptoms of psychopathology in a community sample. Developmental Psychobiology, 57(1), 120-130.

Pelham, W. E., Fabiano, G. A., \& Massetti, G. M. (2005). Evidence-based assessment of attention deficit hyperactivity disorder in children and adolescents. Journal of Clinical Child and Adolescent Psychology, 34(3), 449-476.

Pelham, W. E., Foster, E. M., \& Robb, J. A. (2007). The economic impact of attentiondeficit/hyperactivity disorder in children and adolescents. Journal of Pediatric Psychology, 32(6), 711-727.

Pelham, W. E., Jr., Gnagy, E. M., Greenslade, K. E., \& Milich, R. (1992). Teacher ratings of $D S M-I I I-R$ symptoms for the disruptive behavior disorders. Journal of the American Academy of Child \& Adolescent Psychiatry, 31, 210 -218. doi:10.1097/00004583-199203000-00006 
Pillow, D. R., Pelham, W. E., Hoza, B., Molina, B. S. G., \& Stultz, C. H. (1998). Confirmatory factor analyses examining attention deficit hyperactivity disorder symptoms and other childhood disruptive behaviors. Journal of Abnormal Child Psychology, 26, 293-309. doi:10.1023/A:1022658618368

Polanczyk, G., De Lima, M. S., Horta, B. L., Biederman, J., \& Rohde, L. A. (2007). The worldwide prevalence of ADHD: a systematic review and metaregression analysis. American Journal of Psychiatry, 164(6), 942-948.

Porges, S. W. (1995). Orienting in a defensive world: Mammalian modifications of our evolutionary heritage. A polyvagal theory. Psychophysiology, 32(4), 301-318.

Porges, S. W. (2007). The polyvagal perspective. Biological Psychology, 74(2), 116-143.

Posner, M. I., \& Rothbart, M. K. (2000). Developing mechanisms of selfregulation. Development and Psychopathology, 12(3), 427-441.

Posner, M. I., Rothbart, M. K., Sheese, B. E., \& Voelker, P. (2014). Developing attention: behavioral and brain mechanisms. Advances in Neuroscience, 2014.

Pu, J., Schmeichel, B. J., \& Demaree, H. A. (2010). Cardiac vagal control predicts spontaneous regulation of negative emotional expression and subsequent cognitive performance. Biological Psychology, 84(3), 531-540.

Quigley, K. S., \& Stifter, C. A. (2006). A comparative validation of sympathetic reactivity in children and adults. Psychophysiology, 43(4), 357-365.

Rapport, M. D., Chung, K. M., Shore, G., \& Isaacs, P. (2001). A conceptual model of child psychopathology: Implications for understanding attention deficit hyperactivity disorder and treatment efficacy. Journal of Clinical Child Psychology, 30(1), 48-58.

Rash, J. A., \& Aguirre-Camacho, A. (2012). Attention-deficit hyperactivity disorder and cardiac vagal control: A systematic review. ADHD Attention Deficit and Hyperactivity Disorders, 4, 167-177.

Ratcliff, R. (1979). Group reaction time distributions and an analysis of distribution statistics. Psychological Bulletin, 86(3), 446-461.

Richter, M., \& Gendolla, G. H. E. (2009). The heart contracts to reward: monetary incentives and preejection period. Psychophysiology, 46, 451-457.

https://doi.org/10.1111/j.1469-8986.2009.00795.

Rohde, L. A., Szobot, C., Polanczyk, G., Schmitz, M., Martins, S., \& Tramontina, S. (2005). Attention-deficit/hyperactivity disorder in a diverse culture: do research and clinical findings support the notion of a cultural construct for the disorder? Biological Psychiatry, 57(11), 1436-1441. 
Rothbart, M. K. (2007). Temperament, development, and personality. Current Directions in Psychological Science, 16(4), 207-212.

Rothbart, M. K., Derryberry, D., \& Posner, M. I. (1994). A psychobiological approach to the development of temperament. Temperament: Individual Differences at the Interface of Biology and Behavior, 83-116. Washington, DC: American Psychological Association.

Russell, V. A., Oades, R. D., Tannock, R., Killeen, P. R., Auerbach, J. G., Johansen, E. B., \& Sagvolden, T. (2006). Response variability in attention-deficit/hyperactivity disorder: a neuronal and glial energetics hypothesis. Behavioral and Brain Functions, 2(1), 30.

Ryan, M., Martin, R., Denckla, M. B., Mostofsky, S. H., \& Mahone, E. M. (2010). Interstimulus jitter facilitates response control in children with ADHD. Journal of the International Neuropsychological Society, 16(2), 388-393.

Scheres, A., Oosterlaan, J., \& Sergeant, J. A. (2001). Response execution and inhibition in children with $\mathrm{AD} / \mathrm{HD}$ and other disruptive disorders: The role of behavioural activation. The Journal of Child Psychology and Psychiatry and Allied Disciplines, 42(3), 347-357.

Seidman, L. J., Biederman, J., Faraone, S. V., Weber, W., \& Ouellette, C. (1997). Toward defining a neuropsychology of attention deficit-hyperactivity disorder: Performance of children and adolescents from a large clinically referred sample. Journal of Consulting and Clinical Psychology, 65(1), 150.

Sergeant, J. (2000). The cognitive-energetic model: an empirical approach to attentiondeficit hyperactivity disorder. Neuroscience \& Biobehavioral Reviews, 24(1), 712.

Sergeant, J. A. (2005). Modeling attention-deficit/hyperactivity disorder: a critical appraisal of the cognitive-energetic model. Biological Psychiatry, 57(11), 12481255.

Shaffer, D., Fisher, P., Lucas, C. P., Dulcan, M. K., \& Schwab-Stone, M. E. (2000). NIMH Diagnostic Interview Schedule for Children Version IV (NIMH DISC-IV): description, differences from previous versions, and reliability of some common diagnoses. Journal of the American Academy of Child \& Adolescent Psychiatry, 39(1), 28-38.

Slaats-Willemse, D., Swaab-Barneveld, H., De Sonneville, L., Van Der Meulen, E., \& Buitelaar, J. A. N. (2003). Deficient response inhibition as a cognitive endophenotype of ADHD. Journal of the American Academy of Child \& Adolescent Psychiatry, 42(10), 1242-1248. 
Sonuga-Barke, E. J. (2002). Psychological heterogeneity in AD/HD - a dual pathway model of behaviour and cognition. Behavioural Brain Research, 130(1-2), 29-36.

Sonuga-Barke, E. J. (2005). Causal models of attention-deficit/hyperactivity disorder: from common simple deficits to multiple developmental pathways. Biological Psychiatry, 57(11), 1231-1238.

Sonuga-Barke, E., Bitsakou, P., \& Thompson, M. (2010). Beyond the dual pathway model: evidence for the dissociation of timing, inhibitory, and delay-related impairments in attention-deficit/hyperactivity disorder. Journal of the American Academy of Child \& Adolescent Psychiatry, 49(4), 345-355.

Sonuga-Barke, E. J., \& Castellanos, F. X. (2007). Spontaneous attentional fluctuations in impaired states and pathological conditions: a neurobiological hypothesis. Neuroscience \& Biobehavioral Reviews, 31(7), 977-986.

Sturge-Apple, M. L., Suor, J. H., Davies, P. T., Cicchetti, D., Skibo, M. A., \& Rogosch, F. A. (2016). Vagal tone and children's delay of gratification: differential sensitivity in resource-poor and resource-rich environments. Psychological Science, 27(6), 885-893.

Suess, P. E., Porges, S. W., \& Plude, D. J. (1994). Cardiac vagal tone and sustained attention in school-age children. Psychophysiology, 31, 17-22.

Teicher, M. H., Ito, Y., Glod, C. A., \& Barber, N. I. (1996). Objective measurement of hyperactivity and attentional problems in ADHD. Journal of the American Academy of Child \& Adolescent Psychiatry, 35(3), 334-342.

Tenenbaum, R. B., Musser, E. D., Morris, S., Ward, A. R., Raiker, J. S., Coles, E. K., \& Pelham, W. E. (2018). Response inhibition, response execution, and emotion regulation among children with attention-deficit/hyperactivity disorder. Journal of Abnormal Child Psychology, 1-15.

Thayer, J. F., Hansen, A. L., Saus-Rose E., \& Johnsen, B. H. (2009). Heart rate variability, prefrontal neural function, and cognitive performance: The neurovisceral integration perspective on self-regulation, adaptation, and health. Annals of Behavioral Medicine, 37, 141-153.

Thayer, J. F., \& Lane, R. D. (2000). A model of neurovisceral integration in emotion regulation and dysregulation. Journal of Affective Disorders, 61(3), 201-216.

Thomas, R., Sanders, S., Doust, J., Beller, E., \& Glasziou, P. (2015). Prevalence of attention-deficit/hyperactivity disorder: a systematic review and metaanalysis. Pediatrics, 135(4), e994-e1001. 
Tillman, C. M., Thorell, L. B., Brocki, K. C., \& Bohlin, G. (2007). Motor response inhibition and execution in the stop-signal task: development and relation to ADHD behaviors. Child Neuropsychology, 14(1), 42-59.

Toplak, M. E., West, R. F., \& Stanovich, K. E. (2013). Practitioner review: Do performance-based measures and ratings of executive function assess the same construct?. Journal of Child Psychology and Psychiatry, 54(2), 131-143.

Trommer, B. L., Hoeppner, J. A. B., Lorber, R., \& Armstrong, K. J. (1988). The GoNo-Go paradigm in attention deficit disorder. Annals of Neurology, 24(5), 610614.

Usher, M., \& McClelland, J. L. (2001). The time course of perceptual choice: the leaky, competing accumulator model. Psychological Review, 108(3), 550.

Utendale, W. T., Nuselovici, J., Saint-Pierre, A. B., Hubert, M., Chochol, C., \& Hastings, P. D. (2014). Associations between inhibitory control, respiratory sinus arrhythmia, and externalizing problems in early childhood. Developmental Psychobiology, 56(4), 686-699.

Vaurio, R. G., Simmonds, D. J., \& Mostofsky, S. H. (2009). Increased intra-individual reaction time variability in attention-deficit/hyperactivity disorder across response inhibition tasks with different cognitive demands. Neuropsychologia, 47(12), 2389-2396.

Wahlstedt, C., Thorell, L. B., \& Bohlin, G. (2009). Heterogeneity in ADHD: Neuropsychological pathways, comorbidity and symptom domains. Journal of Abnormal Child Psychology, 37(4), 551-564.

Walcott, C. M., \& Landau, S. (2004). The relation between disinhibition and emotion regulation in boys with attention deficit hyperactivity disorder. Journal of clinical Child and Adolescent Psychology, 33(4), 772-782.

Ward, A. R., Alarcón, G., Nigg, J. T., \& Musser, E. D. (2015). Variation in parasympathetic dysregulation moderates short-term memory problems in childhood attention-deficit/hyperactivity disorder. Journal of Abnormal Child Psychology, 43(8), 1573-1583.

Wechsler, D. (2009). Wechsler Individual Achievement Test-Third Edition. San Antonio, TX: Psychological Corporation.

Wechsler, D., (2011). WASI-II: Wechsler Abbreviated Scale of Intelligence - Second Edition. San Antonio, TX: Pearson

Willcutt, E. G. (2012). The prevalence of DSM-IV attention-deficit/hyperactivity disorder: a meta-analytic review. Neurotherapeutics, 9(3), 490-499. 
Willcutt, E. G., Doyle, A. E., Nigg, J. T., Faraone, S. V., \& Pennington, B. F. (2005). Validity of the executive function theory of attention-Deficit/Hyperactivity disorder: A meta-analytic review. Biological Psychiatry, 57, 1336-1346.

Willcutt, E. G., Nigg, J. T., Pennington, B. F., Solanto, M. V., Rohde, L. A., Tannock, R., ... \& Lahey, B. B. (2012). Validity of DSM-IV attention deficit/hyperactivity disorder symptom dimensions and subtypes. Journal of Abnormal Psychology, 121(4), 991.

Willcutt, E. G., Sonuga-Barke, E. J., Nigg, J. T., \& Sergeant, J. A. (2008). Recent developments in neuropsychological models of childhood psychiatric disorders. In Biological Child Psychiatry (Vol. 24, pp. 195-226). Karger Publishers.

Williams, B. R., Ponesse, J. S., Schachar, R. J., Logan, G. D., \& Tannock, R. (1999). Development of inhibitory control across the life span. Developmental Psychology, 35(1), 205.

Williams, B. R., Strauss, E. H., Hultsch, D. F., Hunter, M. A., \& Tannock, R. (2007). Reaction time performance in adolescents with attention deficit/hyperactivity disorder: Evidence of inconsistency in the fast and slow portions of the RT distribution. Journal of Clinical and Experimental Neuropsychology, 29(3), 277289.

Willoughby, M. T., Wirth, R. J., \& Blair, C. B. (2012). Executive function in early childhood: Longitudinal measurement invariance and developmental change. Psychological Assessment, 24, 418-431.

Wilson, B. A. (1993). Ecological validity of neuropsychological assessment: Do neuropsychological indexes predict performance in everyday activities?. Applied and Preventive Psychology, 2(4), 209-215.

Zelazo, P. D., \& Carlson, S. M. (2012). Hot and cool executive function in childhood and adolescence: Development and plasticity. Child Development Perspectives, 6(4), 354-360. 
APPENDICES 


\section{Study 1 Tables and Figures}

Table 1. Demographic and Diagnostic Characteristics for ADHD and Control Groups Study 1 .

\begin{tabular}{|c|c|c|c|c|}
\hline & & Group & & \\
\hline & $\begin{array}{l}\text { ADHD } \\
(\mathrm{n}=126)\end{array}$ & $\begin{array}{l}\text { Control } \\
(\mathrm{n}=70)\end{array}$ & $\mathrm{F} / \chi^{2}$ & $\eta^{2} / V$ \\
\hline \multicolumn{5}{|l|}{ Demographics } \\
\hline Age in Years & $8.75(1.62)$ & $9.56(1.28)$ & $4.66^{*}$ & .02 \\
\hline Est. IQ ${ }^{a}$ & $98.85(12.14)$ & $102.57(12.93)$ & 3.81 & .02 \\
\hline$\%$ Male & $80.2 \%$ & $57.1 \%$ & $11.81^{* *}$ & .25 \\
\hline \%Hispanic & $88.6 \%$ & $88.7 \%$ & 0.00 & .00 \\
\hline \% Racial Minority & $11.5 \%$ & $22.8 \%$ & $3.69^{*}$ & .15 \\
\hline$\%$ Prev Medicated ${ }^{b}$ & $74.6 \%$ & $0 \%$ & $98.42 * *$ & .71 \\
\hline \multicolumn{5}{|l|}{ Parent Ratings } \\
\hline Inatt. Score ${ }^{c}$ & $2.07(0.65)$ & $0.27(0.36)$ & $404.26 * *$ & .69 \\
\hline Hyp. Score ${ }^{c}$ & $1.74(0.69)$ & $0.22(0.24)$ & $275.12 * *$ & .63 \\
\hline Tot. ADHD score ${ }^{c}$ & $1.91(0.58)$ & $0.25(0.27)$ & $453.49 * *$ & .71 \\
\hline \# Inatt. Symptoms $^{c}$ & $6.52(2.70)$ & $0.28(0.71)$ & $315.59 * *$ & .63 \\
\hline \# Hyp. Symptoms ${ }^{c}$ & $5.29(2.81)$ & $0.21(0.55)$ & $195.81^{* *}$ & .51 \\
\hline \# ODD Symptoms $^{c}$ & $2.97(2.46)$ & $0.07(0.40)$ & $83.90^{* *}$ & .31 \\
\hline$\#$ CD Symptoms ${ }^{c}$ & $0.63(0.98)$ & $0.00(0.00)$ & --- & --- \\
\hline \multicolumn{5}{|l|}{ Teacher Ratings } \\
\hline Inatt. Score c & $2.12(0.77)$ & --- & --- & --- \\
\hline Hyp. Score ${ }^{c}$ & $1.71(0.87)$ & --- & --- & --- \\
\hline Tot. ADHD score ${ }^{c}$ & $1.92(0.70)$ & --- & --- & --- \\
\hline \# Inatt. Symptoms ${ }^{c}$ & $6.21(3.07)$ & --- & --- & --- \\
\hline \# Hyp. Symptoms ${ }^{c}$ & $4.80(3.17)$ & --- & --- & --- \\
\hline \# ODD Symptoms $^{\mathrm{c}}$ & $2.75(2.82)$ & --- & --- & --- \\
\hline \# CD Symptoms $^{\mathrm{c}}$ & $0.91(1.46)$ & --- & --- & --- \\
\hline \multicolumn{5}{|l|}{ Comorbid (\%) } \\
\hline ODD Diagnosis ${ }^{\mathrm{d}}$ & 40.2 & 1.5 & $33.24 * *$ & 0.42 \\
\hline CD Diagnosis ${ }^{\mathrm{d}}$ & 4.1 & 0 & $2.80^{*}$ & 0.12 \\
\hline Anx Diagnosis d,e & 10.4 & 3 & 3.24 & 0.13 \\
\hline Mood Diagnosis d,f & 0.8 & 0 & 0.52 & 0.05 \\
\hline
\end{tabular}

Note. $\mathrm{N}=196 . \eta 2$ = Eta-squared; V = Cramer's V; ODD: Oppositional defiant disorder; Inatt.= DSM-5 Inattentive domain; Hyp.= DSM-5 Hyperactive/Impulsive domain. ${ }^{\mathrm{a}}$ Full-Scale IQ from WASI-II; ${ }^{\mathrm{b}}$ Indicates whether child was prescribed medication for ADHD prior to entering study (all youth medicationfree at time of data collection); ${ }^{\mathrm{c}}$ from parent-reported DBD Rating Scale; ${ }^{\mathrm{d}}$ estimate from parent-reported C-DISC; ${ }^{\mathrm{e}}$ Any Generalized Anxiety, Social Phobia, and Separation Anxiety disorder; ${ }^{\mathrm{f}}$ Either Major Depressive or Dysthimic disorder.*p $<.05 ; * * p<.001$. 
Table 2. Inter-Correlations among Clinical, WM, and RSA Indices

\begin{tabular}{|c|c|c|c|c|c|c|c|}
\hline Variables & 2 (ODD) & $3(\mathrm{ADHD})$ & $4(\mathrm{SS})$ & 5 (RB-RSA) & 6 (Nt RSA) & 7 (SS-RSA) & $8(\Delta \mathrm{RSA})$ \\
\hline 1. Age & -.04 & -.14 & $.28^{*}$ & .00 & -.11 & -.12 & .01 \\
\hline 2. ODD Symptoms & 1.00 & $.65^{*}$ & .00 & -.04 & -.11 & -.06 & .09 \\
\hline 3. Parent-rated ADHD & & 1.00 & -.10 & $-.22^{*}$ & $-.18^{*}$ & -.05 & $.16^{*}$ \\
\hline 4. Spatial Span score & & & 1.00 & -.02 & .05 & -.02 & -.07 \\
\hline 5. RBL RSA & & & & 1.00 & .56 & .64 & .00 \\
\hline 6. Neutral RSA & & & & & 1.00 & $.55^{* *}$ & $-.58^{* *}$ \\
\hline 7. Spatial Span RSA & & & & & & 1.00 & $.37^{* *}$ \\
\hline 8. RSA Change & & & & & & & 1.00 .00 \\
\hline
\end{tabular}


Table 3a. Estimated Marginal Means and ANCOVA Results for WM and RSA Variables.

\begin{tabular}{|c|c|c|c|c|}
\hline \multicolumn{5}{|c|}{ EMM (SE) } \\
\hline & ADHD & Control & $F$ & Partial- $\eta^{2}$ a \\
\hline \multicolumn{5}{|l|}{ WM Task } \\
\hline Span Score ${ }^{a, b}$ & $0.69(0.01)$ & $0.69(0.02)$ & 0.00 & $<.01$ \\
\hline Span Correct Trials ${ }^{c}$ & $3.77(0.20)$ & $3.74(0.34)$ & 0.01 & $<.01$ \\
\hline \multicolumn{5}{|l|}{ RSA } \\
\hline Resting RSA & $6.17(0.13)$ & $7.09(0.22)$ & $9.59^{*}$ & .05 \\
\hline Neutral RSA & $6.25(0.14)$ & $6.90(0.23)$ & $4.51^{*}$ & .03 \\
\hline Span RSA & $6.61(0.12)$ & $6.99(0.20)$ & 2.04 & .01 \\
\hline RSA Change $^{\mathrm{d}}$ & $0.37(0.12)$ & $0.10(0.21)$ & 0.88 & .01 \\
\hline
\end{tabular}

$\overline{\text { Notes. }}{ }^{+}$Estimated Marginal Mean (Standard Error), with covariates: age, gender, previous medication status, ODD symptoms. $\eta_{p}{ }^{2}=$ partial-Eta squared. ${ }^{\mathrm{a}}$ Span Score Range: $.333-.897^{\mathrm{b}}$ Partial-credit scoring (see Methods). ${ }^{\mathrm{c}}$ Number of error-free trials. ${ }^{\mathrm{d}}$ Difference of RSA WM task minus RSA during Neutral period. $* p<.05$.

Table 3b. Raw Means for WM and RSA Variables with Between-Groups Comparisons.

\begin{tabular}{|c|c|c|c|c|}
\hline \multicolumn{5}{|c|}{ Mean (SD) } \\
\hline & ADHD & Control & $F$ & $\eta^{2}$ \\
\hline \multicolumn{5}{|l|}{ WM Score } \\
\hline Span Score ${ }^{\mathrm{a}, \mathrm{b}}$ & $0.69(0.13)$ & $0.70(0.13)$ & 0.41 & $<.01$ \\
\hline Span Correct Trials ${ }^{c}$ & $3.62(1.83)$ & $4.28(2.31)$ & $4.70^{*}$ & .02 \\
\hline \multicolumn{5}{|l|}{ RSA } \\
\hline Resting RSA & $6.30(1.27)$ & $6.86(1.12)$ & $9.47^{*}$ & .05 \\
\hline Neutral RSA & $6.29(1.36)$ & $6.79(0.97)$ & $7.24^{*}$ & .04 \\
\hline Span RSA & $6.69(1.17)$ & $6.87(0.96)$ & 1.28 & .01 \\
\hline RSA Change $^{\mathrm{d}}$ & $0.41(1.20)$ & $0.09(0.98)$ & 0.32 & .02 \\
\hline
\end{tabular}

Notes. ${ }^{\text {a }}$ Span Score Range: .333 - .897 ${ }^{\mathrm{b}}$ Partial-credit scoring (see Methods). ${ }^{\mathrm{c}}$ Number of errorfree trials. ${ }^{\mathrm{d}}$ Difference of RSA WM task minus RSA during Neutral period. ${ }^{*} p<.05$. 
Table 4a. Results of Regression Models Predicting Mean Rating of Overall ADHD Symptoms

\begin{tabular}{lccccc}
\hline Predictor & $t$ & $b$ & $S E$ & $\beta$ & $s r^{2}$ \\
\hline WM Score & -1.10 & -0.39 & .036 & -.06 & .003 \\
Resting RSA & $-3.27^{*}$ & -0.12 & 0.04 & -.16 & .023 \\
Neutral RSA & $-1.95^{\wedge}$ & -0.07 & 0.04 & -.10 & .008 \\
RSA-reactivity & 1.39 & 0.06 & 0.04 & .07 & .004
\end{tabular}

Note. $s r^{2}=$ squared semi-partial correlation; all models included these covariates: sex, child age, ODD symptoms and prescribed medication status. $* p<.05 .{ }^{\wedge} p=.053$

Table 4b. Results of Regression Models Predicting Mean Rating of Inattention Symptoms

\begin{tabular}{lccccc}
\hline Predictor & $t$ & $b$ & $S E$ & $\beta$ & $s r^{2}$ \\
\hline WM Score & -1.11 & -0.38 & 0.34 & -0.05 & .003 \\
Resting RSA & $-4.04^{* *}$ & -0.14 & 0.03 & -0.16 & .026 \\
RSA-reactivity & 0.78 & 0.03 & 0.04 & 0.03 & .001 \\
\hline
\end{tabular}

Note. $s r^{2}=$ squared semi-partial correlation; all models included these covariates: sex, child age, ODD symptoms and prescribed medication status. $* p<.05$. **p $<.001$

Table 4c. Results of Regression Models Predicting Mean Rating of Hyperactive/Impulsive Symptoms

\begin{tabular}{lccccc}
\hline Predictor & $t$ & $b$ & $S E$ & $\beta$ & $s r^{2}$ \\
\hline WM Score & 0.20 & 0.06 & 0.28 & 0.01 & $<.001$ \\
Resting RSA & 1.10 & 0.03 & 0.03 & 0.04 & .002 \\
RSA-reactivity & 0.43 & 0.01 & 0.03 & 0.02 & $<.001$
\end{tabular}

Note. $s r^{2}=$ squared semi-partial correlation; all models included these covariates: sex, child age, ODD symptoms and prescribed medication status. $* p<.05 . * * p<.001$ 
Table 5. Conditional Effects - WM Predicting Mean ADHD Rating by level of RSA-

Change.

\begin{tabular}{|c|c|c|c|c|c|}
\hline Outcome & $\Delta \mathrm{RSA}$ & $\mathrm{b}$ & $\mathrm{SE}$ & $95 \% \mathrm{CI}$ & $\bar{p}$ \\
\hline \multirow{3}{*}{ Overall ADHD Rating } & $-1 \mathrm{SD}$ & 0.41 & 0.48 & $(-0.54,1.35)$ & .40 \\
\hline & Mean & -0.52 & 0.36 & $(-1.23,0.18)$ & .14 \\
\hline & $+1 \mathrm{SD}$ & -1.46 & 0.53 & $(-2.49,-0.42)$ & .01 \\
\hline \multicolumn{6}{|c|}{ Interaction term $p<.01$} \\
\hline \multirow{3}{*}{ IN Rating } & $-1 \mathrm{SD}$ & -0.06 & 0.47 & $(-0.98,0.86)$ & .90 \\
\hline & Mean & -0.46 & 0.35 & $(-1.15,0.22)$ & .19 \\
\hline & $+1 \mathrm{SD}$ & -0.86 & 0.52 & $(-1.89,0.16)$ & .10 \\
\hline \multicolumn{6}{|c|}{ Interaction term $p=.14$} \\
\hline \multirow{3}{*}{ HI Rating } & $-1 \mathrm{SD}$ & 0.34 & 0.38 & $(-0.40,1.09)$ & .37 \\
\hline & Mean & 0.02 & 0.28 & $(-0.54,0.58)$ & .93 \\
\hline & $+1 \mathrm{SD}$ & -0.29 & 0.42 & $(-1.13,0.54)$ & .49 \\
\hline Interaction term $p=.32$ & & & & & \\
\hline
\end{tabular}

Note. IN, Inattentive symptom domain; HI, hyperactive/impulsive symptom domain. Models with IN rating and $\mathrm{HI}$ rating as outcomes included $\mathrm{HI}$ rating and IN rating, respectively, as an additional covariate. 
Figure 1. Interaction of RSA Change and WM Predicting Overall ADHD Raw Score

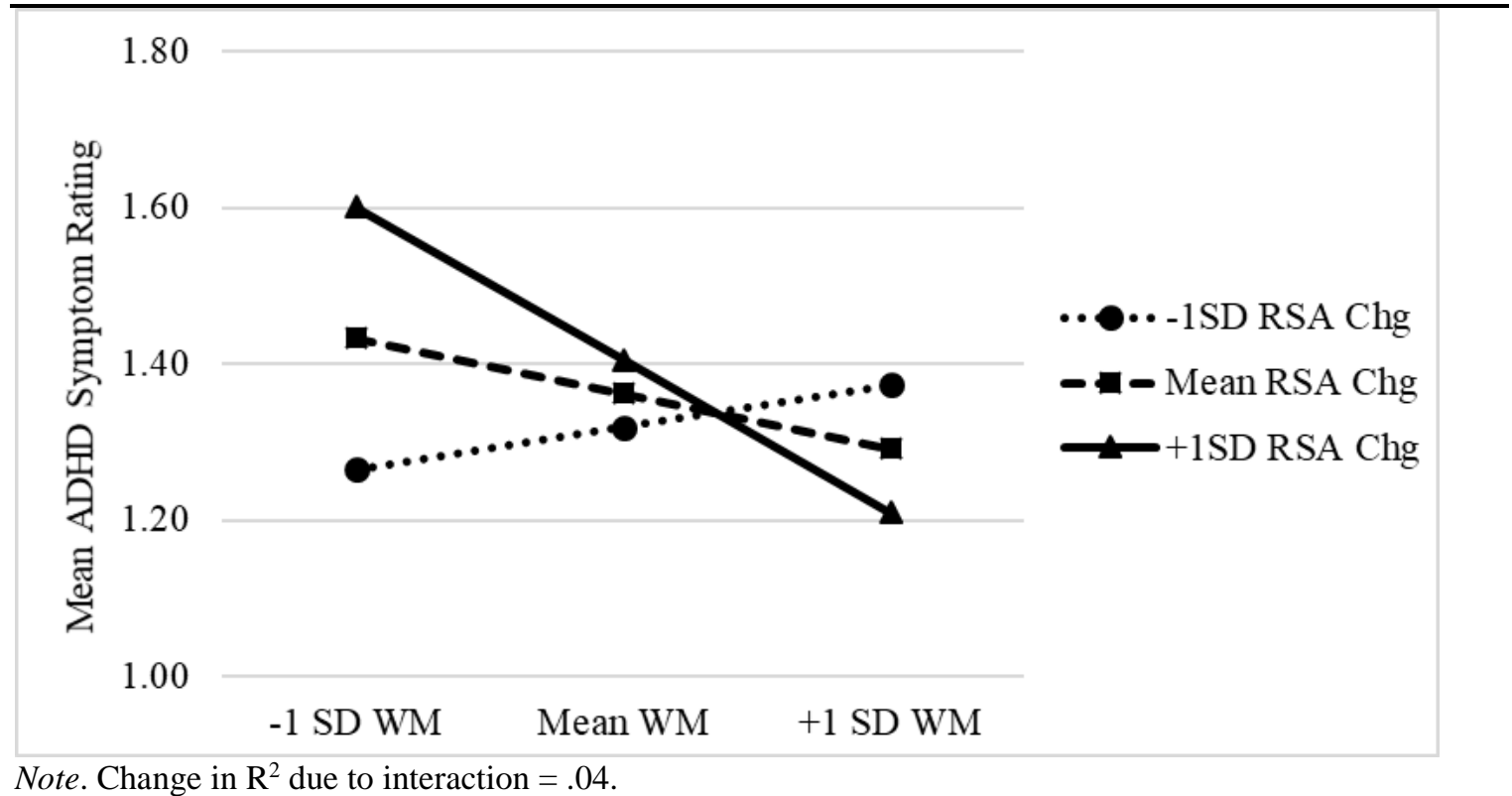

Figure 2. Mean RSA across Each Period per Group - Study 1.

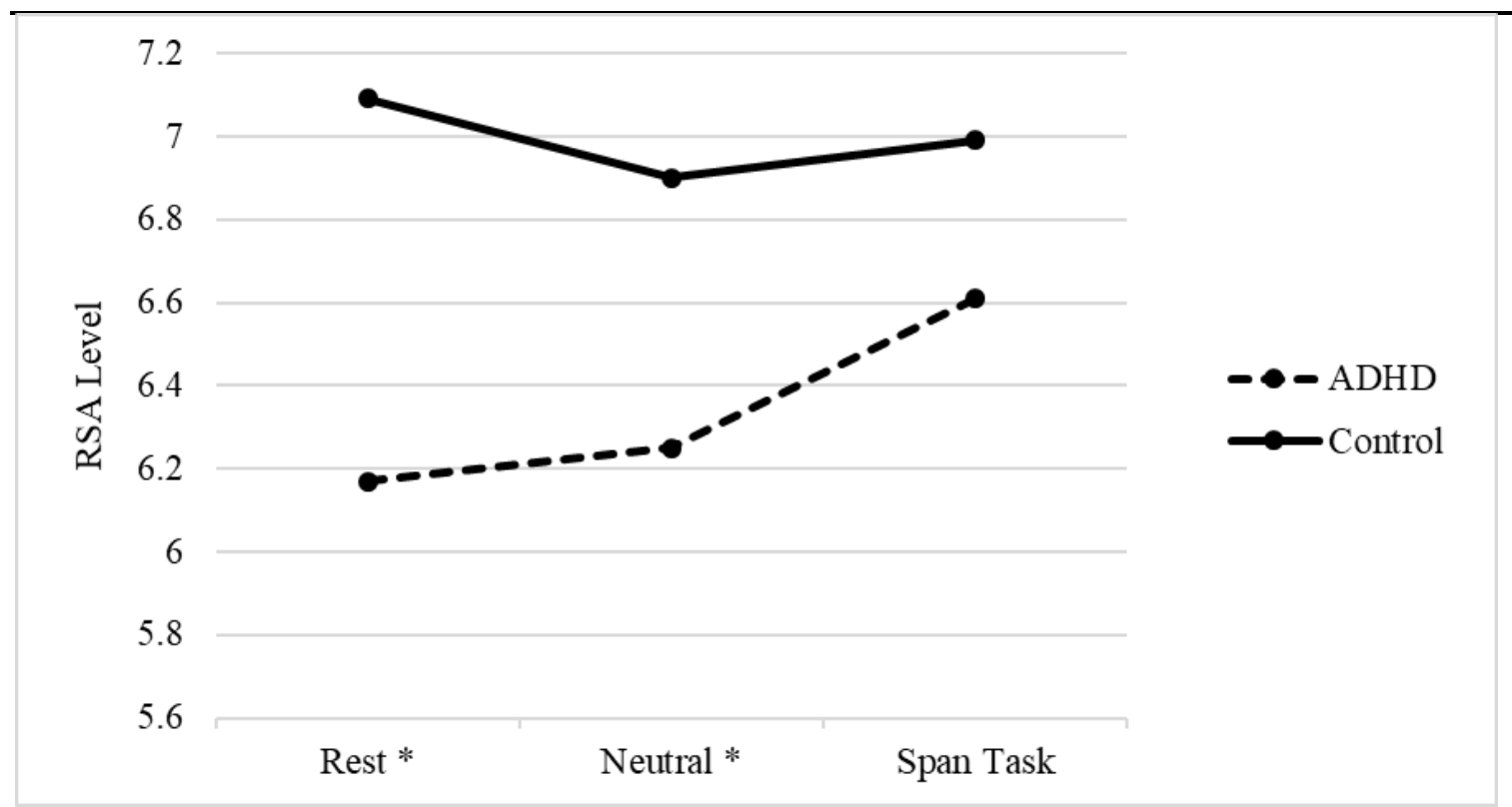

Note. ${ }^{*} p<.05$ for between-group effect. 


\section{Study 2 Tables and Figures}

Table 6. Demographic and Diagnostic Characteristics for ADHD and Control Groups for Study 2.

Demographics

\begin{tabular}{|c|c|c|c|c|}
\hline & $\begin{array}{l}\text { ADHD } \\
(n=70)\end{array}$ & $\begin{array}{l}\text { Group } \\
\text { Control } \\
(n=79)\end{array}$ & $\mathrm{F} / \mathrm{X} 2$ & $\mathrm{Eta}^{2} / \mathrm{V}$ \\
\hline \multicolumn{5}{|l|}{ Demographics } \\
\hline Age (years) & $8.77(1.61)$ & $8.88(1.72)$ & 0.18 & $<.01$ \\
\hline Estimated $\mathrm{IQ}^{\mathrm{a}}$ & $97.91(13.17)$ & $100.71(12.32)$ & 1.76 & .01 \\
\hline$\%$ Male & $79.5 \%$ & $54.8 \%$ & $10.64 *$ & .26 \\
\hline \%Hispanic/Latino & $91.0 \%$ & $88.7 \%$ & 0.20 & .04 \\
\hline$\%$ Racial Minority & $11.6 \%$ & $16.2 \%$ & 0.60 & .07 \\
\hline$\%$ College Educated ${ }^{b}$ & $44.4 \%$ & $69.2 \%$ & $9.72^{*}$ & .26 \\
\hline \%Prev. Medicated ${ }^{\mathrm{g}}$ & $69.9 \%$ & $0 \%$ & $76.80^{* *}$ & .73 \\
\hline \multicolumn{5}{|l|}{ Parent Ratings } \\
\hline Inattention Score & $2.00(0.72)$ & $0.32(0.37)$ & $331.72^{* *}$ & .69 \\
\hline Hyp. Score & $1.80(0.72)$ & $0.26(0.28)$ & $302.17^{* *}$ & .67 \\
\hline Total ADHD Score & $1.90(0.63)$ & $0.29(0.28)$ & $410.33^{* *}$ & .74 \\
\hline \# Inatt. Symptoms ${ }^{c}$ & $6.12(2.77)$ & $0.29(0.75)$ & $347.22^{* *}$ & .69 \\
\hline \# Hyp. Symptoms ${ }^{c}$ & $5.34(2.85)$ & $0.24(0.59)$ & $260.37^{* *}$ & .63 \\
\hline \# ODD Symptoms ${ }^{c}$ & $3.01(2.54)$ & $0.07(0.40)$ & $111.35^{* *}$ & .42 \\
\hline \# CD Symptoms ${ }^{c}$ & $0.67(1.05)$ & 0.00 & $34.46^{* *}$ & .18 \\
\hline \multicolumn{5}{|l|}{ Teacher Ratings } \\
\hline Inattention Score & $2.05(0.82)$ & $0.52(0.71)$ & $39.99^{* *}$ & .32 \\
\hline Hyp. Score & $1.80(0.88)$ & $0.26(0.35)$ & $38.77^{* *}$ & .32 \\
\hline Total ADHD Score & $1.92(0.75)$ & $0.39(0.42)$ & $51.03^{* *}$ & .38 \\
\hline \# Inatt. Symptoms ${ }^{c}$ & $6.15(3.01)$ & $1.15(2.27)$ & $32.37^{* *}$ & .28 \\
\hline \# Hyp. Symptoms ${ }^{c}$ & $5.25(3.09)$ & $0.23(0.83)$ & $33.51^{* *}$ & .29 \\
\hline \# ODD Symptoms ${ }^{c}$ & $2.88(2.70)$ & $0.08(0.28)$ & $13.85^{* *}$ & .14 \\
\hline \# CD Symptoms ${ }^{c}$ & $1.03(1.55)$ & 0.0 & $5.69^{*}$ & .06 \\
\hline \multicolumn{5}{|l|}{ Comorbidity (\%) } \\
\hline ODD Diagnosis ${ }^{\mathrm{d}}$ & 40.6 & 2.7 & $30.48^{* *}$ & .46 \\
\hline CD Diagnosis ${ }^{d}$ & 4.3 & 0 & 3.24 & .15 \\
\hline Anx Diagnosis d,e & 10.0 & 4.2 & 1.85 & .11 \\
\hline Dep Diagnosis d,f & 0 & 0 & -- & -- \\
\hline
\end{tabular}

Note. $N=238$. ODD: Oppositional defiant disorder; Inatt.: DSM-5 Inattentive domain; Hyp.: DSM-5 Hyperactive/Impulsive domain. ${ }^{a}$ Full-Scale IQ from WASI-II; ${ }^{b}$ Parents attaining 4-year college degree or higher; ${ }^{\mathrm{c}}$ from parent-reported DBD Rating Scale; ${ }^{\mathrm{d}}$ estimate from parent-reported C-DISC; ${ }^{\mathrm{e}}$ Summed across Generalized Anxiety, Social Phobia, and Separation Anxiety; ${ }^{\mathrm{f}}$ Summed across Major Depressive and Dysthimic disorders; ${ }^{\mathrm{g}}$ Children prescribed medication for ADHD prior to entering the study (all youth prescribed OROS upon enrolling). $\eta^{2}=$ Eta-squared; $V=$ Cramer's $V .{ }^{*} p<.05 ;{ }^{* *} p<.001$. 
Table 7a. Correlations Among Clinical, Go/No-Go, and RSA Variables - Entire Sample.

\begin{tabular}{|c|c|c|c|c|c|c|c|c|c|}
\hline & 2 & 3 & 4 & 5 & 6 & 7 & 8 & 9 & 10 \\
\hline 1. Age & -.01 & -.10 & $-.18^{*}$ & -.06 & $-.35^{* *}$ & .06 & -.04 & -.10 & -.06 \\
\hline 2. ODD Symptoms & 1.00 & $.71^{* *}$ & -.02 & .10 & .05 & -.11 & -.03 & .01 & .04 \\
\hline 3. Parent-rated ADHD & & 1.00 & .03 & $.20^{*}$ & .14 & $-.26^{*}$ & -.05 & -.03 & .02 \\
\hline 4. GNG Errors-Om & & & 1.00 & $-.47^{* *}$ & $.22^{*}$ & -.02 & $-.18^{*}$ & -.02 & $.20^{*}$ \\
\hline 5. GNG Errors-Co & & & & 1.00 & .01 & -.03 & $.17^{*}$ & .12 & -.08 \\
\hline 6. MRT Go-Trials & & & & & 1.00 & -.13 & -.12 & -.02 & .12 \\
\hline 7. RBL RSA & & & & & & 1.00 & $.58^{* *}$ & $.60^{* *}$ & -.06 \\
\hline 8. Neutral RSA & & & & & & & 1.00 & $.60^{* *}$ & $-.56^{* *}$ \\
\hline 9. Go/No-Go RSA & & & & & & & & 1.00 & $.31^{* *}$ \\
\hline 10. RSA Change & & & & & & & & & 1.00 \\
\hline
\end{tabular}

Note. $* p<.05 . * * p<.001 . \mathrm{RBL}=$ Resting baseline; $\mathrm{RSA}=$ respiratory sinus arrhythmia. 
Table 7b. Correlations Among Clinical, Go/No-Go, and RSA Variables-ADHD Sample Only ${ }^{\text {. }}$

\begin{tabular}{|c|c|c|c|c|c|c|c|c|c|}
\hline & 2 & 3 & 4 & 5 & 6 & 7 & 8 & 9 & 10 \\
\hline 1. Age & .10 & -.13 & $-.31^{*}$ & .11 & $-.37^{*}$ & .01 & -.01 & -.03 & -.02 \\
\hline 2. ODD Symptoms & 1.00 & $.46^{* *}$ & -.17 & .12 & -.03 & .12 & .13 & .09 & -.09 \\
\hline 3. Parent-rated ADHD & & 1.00 & -.13 & $.32^{*}$ & .15 & -.16 & .16 & .14 & -.06 \\
\hline 4. GNG Errors-Om & & & 1.00 & $-.65^{* *}$ & .23 & -.04 & -.16 & -.12 & .09 \\
\hline 5. GNG Errors-Co & & & & 1.00 & -.02 & .07 & .17 & .13 & -.11 \\
\hline 6. RT Go-Trials & & & & & 1.00 & $-.25^{*}$ & -.24 & -.16 & .14 \\
\hline 7. RBL RSA & & & & & & 1.00 & $.64^{* *}$ & $.52^{* *}$ & $-.30^{*}$ \\
\hline 8. Neutral RSA & & & & & & & 1.00 & $.74^{* *}$ & $-.57^{* *}$ \\
\hline 9. Go/No-Go RSA & & & & & & & & 1.00 & .14 \\
\hline 10. RSA Change & & & & & & & & & 1.00 \\
\hline
\end{tabular}

Note. ${ }^{a}$ ADHD subsample, $\mathrm{n}=70 .{ }^{*} p<.05 .{ }^{* *} p<.001 . \mathrm{RBL}=$ Resting baseline; RSA $=$ respiratory sinus arrhythmia. 
Table 8a. Estimated Marginal Means and ANCOVA Results for Go/No-Go and RSA Variables.

\begin{tabular}{|c|c|c|c|c|}
\hline \multicolumn{5}{|c|}{ EMM (SE) } \\
\hline & ADHD & Control & $F$ & Partial- $\eta^{2 \text { a }}$ \\
\hline \multicolumn{5}{|l|}{ Go/No-Go Task } \\
\hline Errors Omission & $12.92(0.59)$ & $11.57(0.62)$ & 1.73 & .01 \\
\hline Errors Commiss & $6.78(0.37)$ & $6.78(0.38)$ & 0.00 & .00 \\
\hline MeanRT $(\text { go })^{\mathrm{b}}$ & $641.80(25.55)$ & $542.35(26.69)$ & $5.01^{*}$ & .04 \\
\hline Total Errors ${ }^{c}$ & $19.70(0.54)$ & $18.35(0.56)$ & 2.09 & .02 \\
\hline \multicolumn{5}{|l|}{ RSA } \\
\hline Resting RSA & $6.07(0.18)$ & $7.14(0.19)$ & $11.88^{*}$ & .09 \\
\hline Neutral RSA & $6.09(0.20)$ & $6.85(0.20)$ & $4.90^{*}$ & .04 \\
\hline G/NG RSA & $6.66(0.17)$ & $7.00(0.18)$ & 1.32 & .01 \\
\hline RSA Change $^{\mathrm{d}}$ & $0.56(0.17)$ & $0.16(0.17)$ & 1.99 & .02 \\
\hline
\end{tabular}

Note. Estimated marginal mean and standard error with age, gender previous medication status and ODD symptoms as covariates. ${ }^{*} p<.05,{ }^{* *} p<.001 .{ }^{\text {a }}$ Partial- $\eta^{2}$ of $.0099, .0588$, and .1379 reflect small, medium, and large effect sizes, respectively (Cohen, 1969; Richardson, 2011). ${ }^{\mathrm{b}}$ Mean reaction time during the "go" trials. ${ }^{\mathrm{c}}$ Sum of correct hits and correct omissions. ${ }^{\mathrm{d}}$ positive values indicate elevated RSA during the GNG task compared to neutral period.

Table 8b. Raw Means for Go/No-Go and RSA Variables with Between-Groups Comparison.

\begin{tabular}{|c|c|c|c|c|}
\hline \multicolumn{5}{|c|}{ Mean (SD) } \\
\hline & ADHD & Control & $F$ & $\eta^{2}$ \\
\hline \multicolumn{5}{|l|}{ Go/No-Go Task } \\
\hline Errors Omission & 12.63 (3.99) & $11.62(3.39)$ & 2.78 & .02 \\
\hline Errors Commiss & $6.91(2.41)$ & $6.66(2.18)$ & 0.46 & .00 \\
\hline MeanRT $^{a}$ & 612.53 (172.98) & $574.58(168.03)$ & 1.84 & .01 \\
\hline Total Errors ${ }^{b}$ & $19.54(3.04)$ & $18.28(3.48)$ & $5.52^{*}$ & .04 \\
\hline \multicolumn{5}{|l|}{ RSA } \\
\hline Resting RSA & $6.33(1.13)$ & $6.89(1.13)$ & $8.83^{*}$ & .06 \\
\hline Neutral RSA & $6.33(0.15)$ & $6.70(0.13)$ & 3.39 & .02 \\
\hline G/NG RSA & $6.76(0.13)$ & $6.89(0.12)$ & 0.62 & .00 \\
\hline RSA Change $^{\mathrm{c}}$ & $0.41(0.12)$ & $0.19(0.11)$ & 1.68 & .01 \\
\hline
\end{tabular}

Note. ${ }^{*} p<.05,{ }^{a}$ Mean reaction time during the "go" trials. ${ }^{\mathrm{b}}$ Sum of correct hits and correct omissions. ${ }^{\mathrm{c}}$ Positive values indicate elevated RSA during the GNG task compared to neutral period. 
Table 9a. Results of Regression Models Predicting Mean Rating for ADHD Symptoms - Study 2.

\begin{tabular}{|c|c|c|c|c|c|}
\hline Predictor & $t$ & $b$ & $S E$ & $\beta$ & $s r^{2}$ \\
\hline \multicolumn{6}{|l|}{ Go/No-Go Predictors } \\
\hline Errors Commission & $2.01^{*}$ & 0.05 & 0.02 & 0.12 & .013 \\
\hline Errors Omission & 0.46 & 0.01 & 0.02 & 0.03 & .001 \\
\hline Total Errors ${ }^{a}$ & $1.87^{\wedge}$ & 0.03 & 0.02 & 0.11 & .011 \\
\hline Mean RT (go) & $2.24^{*}$ & $<0.01$ & $<0.01$ & 0.14 & .016 \\
\hline \multicolumn{6}{|l|}{ Physiological Predictors } \\
\hline Rest Baseline RSA & $-3.76^{* *}$ & -0.17 & 0.05 & -0.21 & .043 \\
\hline Neutral Period RSA & -0.82 & -0.04 & 0.05 & -0.05 & .002 \\
\hline G/NG Task RSA & -0.88 & -0.05 & 0.05 & -0.05 & .003 \\
\hline RSA Change & 0.12 & 0.01 & 0.06 & 0.01 & $<.001$ \\
\hline
\end{tabular}

Note. $s r^{2}=$ semi-partial correlation. ${ }^{*} p<.05 .{ }^{* *} p<.001 .{ }^{\wedge} p=.06 .{ }^{\text {a }}$ The sum of errors of commission and errors of omission.

Table 9b. Results of Regression Models Predicting Mean Rating for Inattentive Symptoms.

\begin{tabular}{|c|c|c|c|c|c|}
\hline Predictor & $t$ & $b$ & $S E$ & $\beta$ & $s r^{2}$ \\
\hline \multicolumn{6}{|l|}{ Go/No-Go Predictors } \\
\hline Errors Commission & $2.98^{*}$ & 0.06 & 0.02 & 0.14 & .018 \\
\hline Errors Omission & -0.42 & -0.01 & 0.01 & -0.02 & $<.001$ \\
\hline Total Errors ${ }^{a}$ & 1.51 & 0.02 & 0.01 & 0.07 & .005 \\
\hline Mean RT (go) & 1.25 & $<0.01$ & $<0.01$ & 0.06 & .003 \\
\hline \multicolumn{6}{|l|}{ Physiological Predictors } \\
\hline Rest Baseline RSA & $-2.54^{*}$ & -0.11 & 0.04 & -0.12 & .013 \\
\hline Neutral Period RSA & -1.25 & -0.05 & 0.04 & -0.06 & .004 \\
\hline G/NG Task RSA & -0.71 & -0.03 & 0.05 & -0.03 & .001 \\
\hline RSA Change & 0.80 & 0.04 & 0.05 & 0.04 & .002 \\
\hline
\end{tabular}

Note. $s r^{2}=$ semi-partial correlation. ${ }^{*} p<.05 .{ }^{* * *} p<.001$. Each predictor in a separate model; Parent-reported hyperactive/impulsive symptoms covariate. ${ }^{a}$ The sum of errors of commission and errors of omission.

Table 9c. Results of Regression Models Predicting Mean Rating for Hyperactive/Impulsive Symptoms.

\begin{tabular}{rcccccc}
\hline Predictor & $t$ & $b$ & $S E$ & $\beta$ & $p$ & $s r^{2}$ \\
\hline Go/No-Go Predictors & & & & & & \\
Errors Commission & -1.50 & -0.03 & 0.02 & -0.06 & .14 & .003 \\
Errors Omission & 0.81 & 0.01 & 0.01 & 0.03 & .42 & .001 \\
$\quad$ Total Errors ${ }^{2}$ & -0.09 & $<0.01$ & 0.01 & $<0.01$ & .93 & $<.001$ \\
Mean RT (go) & 0.47 & $<0.01$ & $<0.01$ & 0.02 & .64 & $<.001$ \\
Physiological Predictors & & & & & & \\
Rest Baseline RSA & -0.30 & -0.01 & 0.04 & -0.01 & .77 & $<.001$ \\
Neutral Period RSA & 0.66 & 0.02 & 0.03 & 0.03 & .51 & .001 \\
G/NG Task RSA & 0.05 & $<0.01$ & 0.04 & $<0.01$ & .96 & $<.001$ \\
RSA Change & -0.74 & -0.03 & 0.04 & -0.03 & .46 & .001 \\
\hline
\end{tabular}

Note. $s r^{2}=$ semi-partial correlation. ${ }^{*} p<.05 .{ }^{* *} p<.001$. Each predictor in a separate model; Parent-reported inattentive symptoms covariate. ${ }^{\text {a }}$ The sum of errors of commission and errors of omission.. 
Table 10. Conditional Effects - Total GNG Errors predicting Mean ADHD Rating, by level of RSA-Change ${ }^{a}$.

\begin{tabular}{lccccc}
\hline Outcome & $\Delta$ RSA & $b$ & $S E$ & $95 \% C I$ & $p$ \\
\hline \multirow{2}{*}{ Overall ADHD Rating } & $-1 \mathrm{SD}$ & -0.02 & 0.04 & $-0.11-0.06$ & .63 \\
& Mean & 0.01 & 0.03 & $-0.04-0.07$ & .59 \\
& $+1 \mathrm{SD}$ & 0.05 & 0.04 & $-0.02-0.12$ & .16 \\
Interaction term, $p=.21$ & & & & & \\
\hline \multirow{2}{*}{ IN Rating $\mathrm{bc}$} & $-1 \mathrm{SD}$ & -0.06 & 0.05 & $-0.15-0.04$ & .23 \\
& Mean & 0.01 & 0.03 & $-0.05-0.07$ & .83 \\
& $+1 \mathrm{SD}$ & 0.07 & 0.04 & $-0.01-0.15$ & .08 \\
Interaction term, $p=.04$ & & & & & \\
\hline \multirow{2}{*}{ HI Rating ${ }^{\mathrm{d}}$} & $-1 \mathrm{SD}$ & -0.03 & 0.04 & $-0.11-0.05$ & .43 \\
& Mean & -0.01 & 0.02 & $-0.06-0.04$ & .75 \\
Interaction term, $p=.39$ & $+1 \mathrm{SD}$ & 0.01 & 0.03 & $-0.05-0.08$ & .65 \\
\hline
\end{tabular}

${ }^{a}$ In the ADHD subsample only, $\mathrm{n}=70 .{ }^{b}$ Hyperactivity rating included as covariate. ${ }^{\mathrm{c}} R^{2}$ change due to interaction $=.05 . \quad{ }^{\mathrm{d}}$ Inattention rating included as covariate. 
Figure 3. Interaction of RSA Change and GNG Errors Predicting Mean Inattentive Symptom Rating.

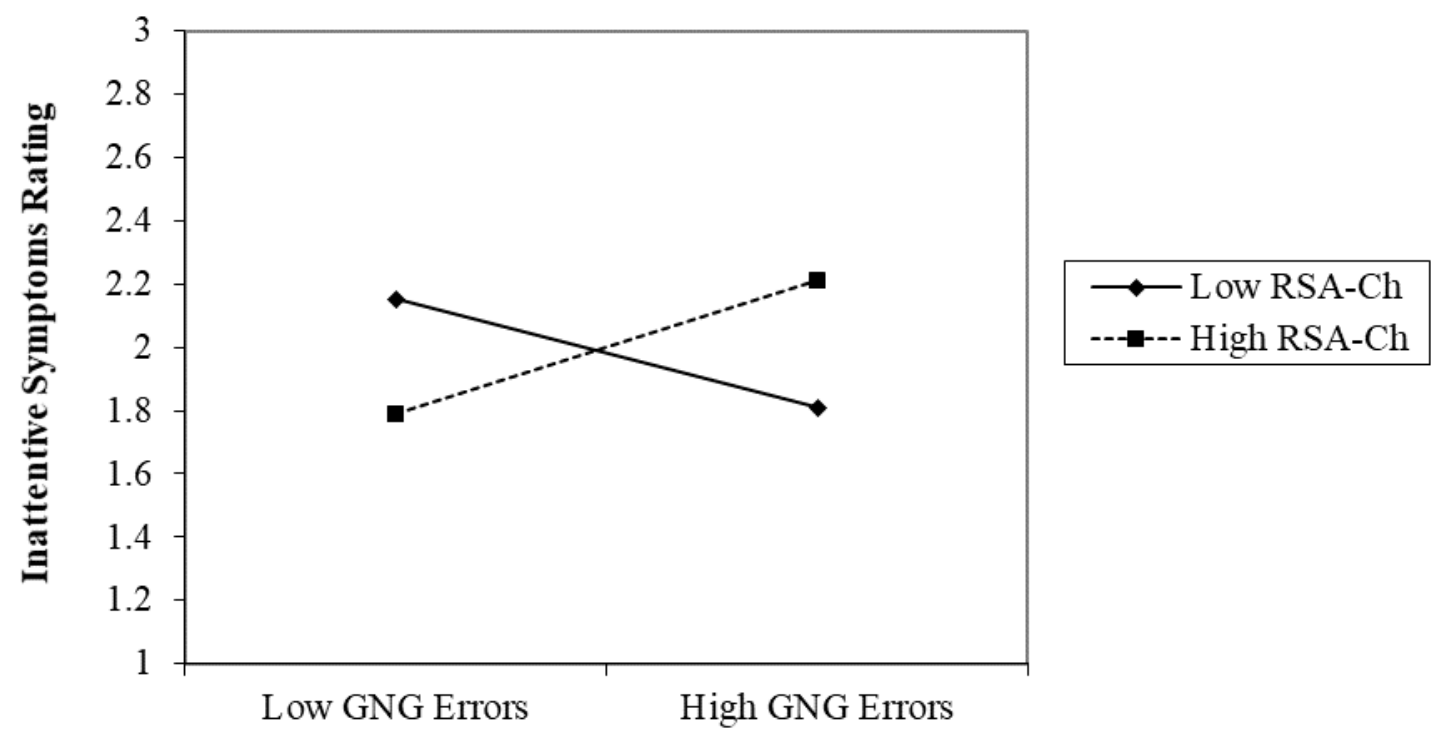

Change in $R^{2}$ due to interaction $=.05$

Note. In the ADHD subsample only, $\mathrm{n}=70$. Mean inattentive symptom rating is from the parent-report DBD-RS rated on a 0-3 scale. Low/High GNG Errors and RSA-Change represent -/+1 SD. 


\section{Study 3 Tables and Figures}

Table 11a. Correlations Among Clinical, RTV, and RSA Variables - Entire Sample.

\begin{tabular}{|c|c|c|c|c|c|c|c|c|c|}
\hline & 2. & 3. & 4. & 5. & 6. & 7. & 8. & 9. & 10. \\
\hline 1. Age & -.10 & $-.35^{* *}$ & $-.35^{* *}$ & -.13 & $-.17^{*}$ & $-.30^{* * *}$ & .06 & -.10 & -.06 \\
\hline 2. ADHD Rating & 1.00 & .14 & .15 & .06 & .10 & .11 & $-.26^{*}$ & -.03 & .02 \\
\hline 3. MRT & & 1.00 & $.75^{* *}$ & $.68^{* *}$ & $.31^{* *}$ & $.63^{* *}$ & -.12 & -.11 & .12 \\
\hline 4. SDRT & & & 1.00 & $.20^{*}$ & $.32^{* *}$ & $.88^{* *}$ & -.08 & .10 & $.19^{*}$ \\
\hline 5. $\mathrm{Mu}$ & & & & 1.00 & $.40^{* *}$ & -.08 & -.12 & -.11 & .02 \\
\hline 6. Sigma & & & & & 1.00 & -.05 & -.01 & .01 & .02 \\
\hline 7. Tau & & & & & & 1.00 & -.05 & .08 & .17 \\
\hline 9. Go/No-Go RSA & & & & & & & & 1.00 & $.31^{* *}$ \\
\hline 10. RSA Change & & & & & & & & & 1.00 \\
\hline
\end{tabular}

Note. MRT= Mean reaction time to "go" trials. SDRT=Standard deviation of reaction time to "go trials. $\Delta$ RSA=change in RSA from neutral period to task period.

Table 11b. Correlations Among Clinical, RTV, and RSA Variables within ADHD Group ${ }^{\mathrm{a}}$

\begin{tabular}{|c|c|c|c|c|c|c|c|c|c|}
\hline & 2. & 3. & 4. & 5. & 6. & 7. & 8. & 9. & 10. \\
\hline 1. Age & -.13 & $-.37^{*}$ & $-.30^{*}$ & -.07 & -.08 & $-.30^{*}$ & .10 & -.03 & -.02 \\
\hline 2. ADHD Rating & 1.00 & .15 & .07 & .16 & .22 & .01 & -.16 & .14 & -.06 \\
\hline 3. MRT & & 1.00 & $.69^{* *}$ & $.62^{* *}$ & .06 & $.65^{* *}$ & $-.25^{*}$ & -.16 & .14 \\
\hline 4. SDRT & & & 1.00 & .04 & .06 & $.93^{* *}$ & -.18 & .01 & .22 \\
\hline 5. $\mathrm{Mu}$ & & & & 1.00 & .20 & -.13 & -.14 & -.18 & .04 \\
\hline 6. Sigma & & & & & 1.00 & -.15 & .08 & -.04 & .03 \\
\hline 7. Tau & & & & & & 1.00 & -.17 & -.02 & .17 \\
\hline 9. Go/No-Go RSA & & & & & & & & 1.00 & .14 \\
\hline 10. RSA Change & & & & & & & & & 1.00 \\
\hline
\end{tabular}

Note. MRT= Mean reaction time to "go" trials. SDRT=Standard deviation of reaction time to "go trials. $\Delta$ RSA=change in RSA from neutral period to task period. ${ }^{a}$ ADHD subsample, $n=70$. 
Table 12a. Estimated Marginal Means and ANCOVA Results for RTV Variables.

\begin{tabular}{|c|c|c|c|c|}
\hline \multicolumn{5}{|c|}{ EMM (SE) } \\
\hline & ADHD & Control & $F$ & $\eta_{p}^{2}$ \\
\hline \multicolumn{5}{|l|}{ RT Distribution Measures } \\
\hline MRT & $641.79(25.55)$ & $542.35(26.69)$ & $5.01^{*}$ & .04 \\
\hline SDRT & $128.51(12.21)$ & $78.12(12.91)$ & $5.56^{*}$ & .043 \\
\hline $\mathrm{Mu}$ & $449.92(19.42)$ & $432.92(20.52)$ & 0.25 & .002 \\
\hline Sigma & $67.33(12.42)$ & $56.46(13.12)$ & 0.25 & .002 \\
\hline Tau & $194.42(21.94)$ & $100.43(23.19)$ & $5.99^{*}$ & .046 \\
\hline \multicolumn{5}{|l|}{ RSA } \\
\hline $\begin{array}{l}\text { Resting RSA } \\
\text { Neutral RSA }\end{array}$ & $\begin{array}{l}0.0 /(0.18) \\
6.09(0.20)\end{array}$ & $\begin{array}{l}1.14(0.19) \\
6.85(0.20)\end{array}$ & $\begin{array}{r}11.88 \\
4.90^{*}\end{array}$ & .09 \\
\hline G/NG RSA & $6.66(0.17)$ & $7.00(0.18)$ & 1.32 & .01 \\
\hline RSA Change $^{b}$ & $0.56(0.17)$ & $0.16(0.17)$ & 1.99 & .02 \\
\hline \multicolumn{5}{|c|}{$\begin{array}{l}\text { Note. Estimated marginal mean and standard error with age, gender previous medication status and ODD } \\
\text { symptoms as covariates. MRT and RSA variables were examined in Study } 2 \text { but are presented here to aid } \\
\text { description. }{ }^{*} p<.05,{ }^{* *} p<.001 .{ }^{\text {a }} \text { Partial- } \eta^{2} \text { of } .0099, .0588 \text {, and } .1379 \text { reflect small, medium, and large } \\
\text { effect sizes, respectively (Cohen, } 1969 ; \text { Richardson, 2011). }{ }^{b} \text { Positive values indicate elevated RSA during } \\
\text { he GNG task compared to neutral period. }\end{array}$} \\
\hline
\end{tabular}

Table 12b. Raw Means for RT and RTV Variables with Between-Groups Comparison.

\begin{tabular}{|c|c|c|c|c|}
\hline \multicolumn{5}{|c|}{ Mean (SD) } \\
\hline & ADHD & Control & $F$ & $\eta^{2}$ \\
\hline \multicolumn{5}{|l|}{ RT Distribution Measures } \\
\hline MRT & $612.53(172.98)$ & $574.58(168.03)$ & 1.84 & .012 \\
\hline SDRT & $115.55(88.26)$ & $89.35(65.08)$ & $4.28^{*}$ & .028 \\
\hline $\mathrm{Mu}$ & $441.21(123.27)$ & $443.24(118.81)$ & 0.01 & $<.001$ \\
\hline Sigma & $64.09(64.68)$ & $57.09(83.35)$ & 0.32 & .002 \\
\hline Tau & $168.46(163.73)$ & $126.33(108.40)$ & $3.47^{+}$ & .023 \\
\hline \multicolumn{5}{|l|}{ RSA } \\
\hline Resting RSA & $6.33(1.13)$ & $6.89(1.13)$ & $8.83^{*}$ & .06 \\
\hline Neutral RSA & $6.33(0.15)$ & $6.70(0.13)$ & 3.39 & .02 \\
\hline G/NG RSA & $6.76(0.13)$ & $6.89(0.12)$ & 0.62 & .00 \\
\hline RSA Change $^{\mathrm{b}}$ & $0.41(0.12)$ & $0.19(0.11)$ & 1.68 & .01 \\
\hline
\end{tabular}

Note. MRT and RSA variables were examined in Study 2 but are presented here to aid description. ${ }^{*} p<.05$. ${ }^{+} p=.06 .{ }^{\text {a }}$ Positive values indicate elevated RSA during the GNG task compared to neutral period. 
Table 13a. Results of Regression Models Predicting Mean Rating for ADHD Symptoms Study 3.

\begin{tabular}{lcccccc}
\hline Predictor & & $t$ & $b$ & $S E$ & $\beta$ & $s r^{2}$ \\
\hline Reaction Time Variability & & & & & & \\
& SDRT & $2.076^{*}$ & 0.001 & 0.001 & 0.125 & .014 \\
Mu & 0.904 & $<0.001$ & $<0.001$ & 0.054 & .003 \\
& Sigma & 0.855 & 0.001 & 0.001 & 0.051 & .002 \\
Tau & $1.955^{\wedge}$ & 0.001 & $<0.001$ & 0.116 & .012
\end{tabular}

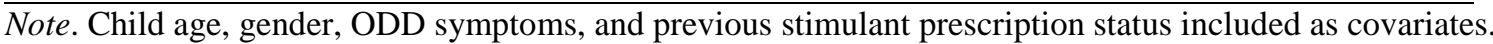
$s r^{2}=$ semi-partial correlation. ${ }^{*} p<.05 .{ }^{* *} p<.001 .{ }^{\wedge} p=.053 .{ }^{\text {a }}$

Table 13b. Results of Regression Models Predicting Mean Rating for Inattentive Symptoms - Study 3.

\begin{tabular}{lcccccc}
\hline Predictor & & $t$ & $b$ & $S E$ & $\beta$ & $s r^{2}$ \\
\hline Reaction Time Variability & & & & & & \\
& SDRT & 1.687 & 0.001 & 0.001 & 0.084 & .008 \\
& $\mathrm{Mu}$ & 0.314 & $<0.001$ & $<0.001$ & 0.015 & $<.001$ \\
& Sigma & -0.402 & $<0.001$ & 0.001 & -0.020 & $<.001$ \\
& Tau & 1.722 & 0.001 & $<0.001$ & 0.084 & .006 \\
\hline
\end{tabular}

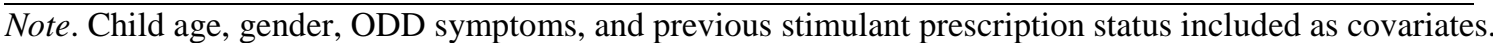
$s r^{2}=$ semi-partial correlation. ${ }^{*} p<.05 .{ }^{* *} p<.001 .^{\mathrm{a}}$.

Table 13c. Results of Regression Models Predicting Mean Rating for Hyperactive/Impulsive Symptoms - Study 3.

\begin{tabular}{lcccccc}
\hline Predictor & & $t$ & $b$ & $S E$ & $\beta$ & $s r^{2}$ \\
\hline Reaction Time Variability & & & & & & \\
& SDRT & -0.108 & $<0.001$ & 0.001 & -0.005 & $<.001$ \\
Mu & 0.389 & $<0.001$ & $<0.001$ & 0.016 & $<.001$ \\
& Sigma & 1.103 & 0.001 & $<0.001$ & 0.044 & .002 \\
Tau & -0.241 & $<0.001$ & $<0.001$ & -0.010 & $<.001$ \\
\hline
\end{tabular}

Note. Child age, gender, ODD symptoms, previous stimulant prescription status, and parent inattention rating included as covariates. $* p<.05$. ${ }^{\text {a }}$ The sum of errors of commission and errors of omission. 
Table 14a. Conditional Effects - SDRT predicting ADHD Symptom Severity, by level of RSAChange.

\begin{tabular}{|c|c|c|c|c|c|}
\hline Outcome & $\Delta \mathrm{RSA}$ & $b$ & $S E$ & $95 \% C I$ & $p$ \\
\hline \multirow{3}{*}{ Overall ADHD Rating } & $-1 \mathrm{SD}$ & $<-0.001$ & 0.001 & $-0.003,0.002$ & .93 \\
\hline & Mean & 0.001 & 0.001 & $<-0.001,0.003$ & .10 \\
\hline & $+1 \mathrm{SD}$ & 0.003 & 0.001 & $0.001,0.004$ & $<.001$ \\
\hline \multicolumn{6}{|l|}{ Interaction term, $p=.053$} \\
\hline \multirow{3}{*}{ IN Rating ${ }^{a}$} & $-1 \mathrm{SD}$ & -0.001 & 0.001 & $-0.003,0.001$ & .28 \\
\hline & Mean & 0.001 & 0.001 & $-0.001,0.002$ & .44 \\
\hline & $+1 \mathrm{SD}$ & 0.002 & 0.001 & $0.001,0.004$ & $<.01$ \\
\hline \multicolumn{6}{|l|}{ Interaction term, $p<.01$} \\
\hline \multirow{3}{*}{ HI Rating ${ }^{b}$} & $-1 \mathrm{SD}$ & 0.001 & 0.001 & $-0.001,0.002$ & .29 \\
\hline & Mean & $<0.001$ & 0.001 & $-0.001,0.001$ & .60 \\
\hline & $+1 \mathrm{SD}$ & $<-0.001$ & 0.001 & $-0.002,0.001$ & .63 \\
\hline Interaction term, $p=.24$ & & & & & \\
\hline
\end{tabular}

Table 14b. Conditional Effects - Tau predicting ADHD Symptom Severity, by level of RSAChange.

\begin{tabular}{|c|c|c|c|c|c|}
\hline Outcome & $\Delta \mathrm{RSA}$ & $b$ & $S E$ & $95 \% C I$ & $p$ \\
\hline \multirow{3}{*}{ Overall ADHD Rating } & $-1 \mathrm{SD}$ & $<0.001$ & 0.001 & $-0.001,0.001$ & .94 \\
\hline & Mean & 0.001 & $<0.001$ & $-0.001,0.002$ & .07 \\
\hline & $+1 \mathrm{SD}$ & 0.002 & 0.001 & $0.001,0.003$ & $<.01$ \\
\hline \multicolumn{6}{|l|}{ Interaction term, $p=.08$} \\
\hline \multirow{3}{*}{ IN Rating a } & $-1 \mathrm{SD}$ & $<0.001$ & 0.001 & $-0.001,0.001$ & .62 \\
\hline & Mean & 0.001 & $<0.001$ & $<0.001,0.001$ & .22 \\
\hline & $+1 \mathrm{SD}$ & 0.001 & 0.001 & $0.001,0.002$ & .01 \\
\hline \multicolumn{6}{|l|}{ Interaction term, $p=.04$} \\
\hline \multirow{3}{*}{ HI Rating ${ }^{b}$} & $-1 \mathrm{SD}$ & $<0.001$ & 0.001 & $-0.001,0.001$ & .56 \\
\hline & Mean & $<0.001$ & $<0.001$ & $-0.001,0.001$ & .85 \\
\hline & $+1 \mathrm{SD}$ & $<0.001$ & $<0.001$ & $-0.001,0.001$ & .69 \\
\hline
\end{tabular}

$\overline{\text { Note. Child age, gender, ODD symptoms, and prior stimulant medication included as covariates in model. }}{ }^{a}$ Hyperactivity rating also included as covariate. ${ }^{\mathrm{b}}$ Inattention rating also included as covariate. 
Figure 4. ex-Gaussian Probability Function

A

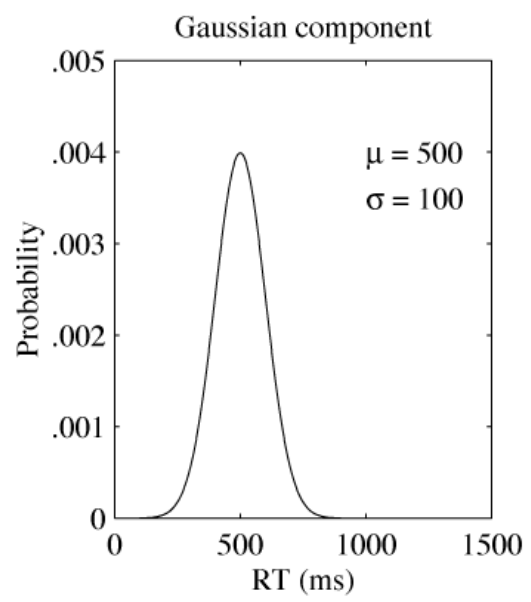

B

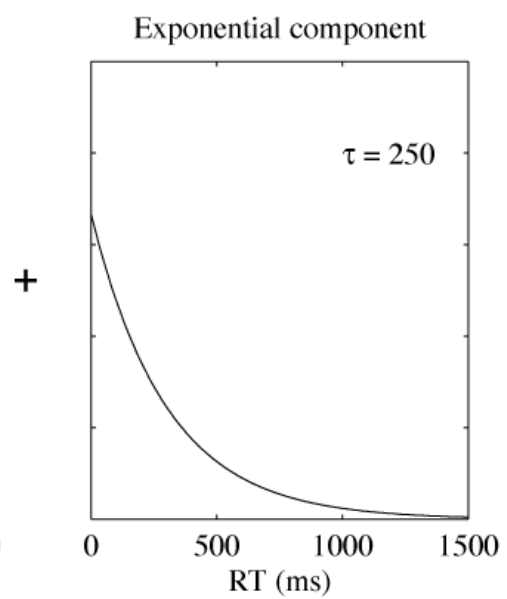

C

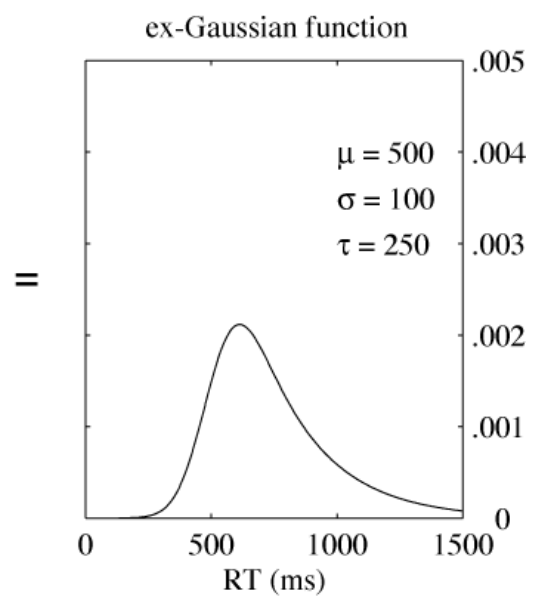

Note. The ex-Gaussian probability function, shown in panel C, as a convolution of (A) a normally distributed (i.e., Gaussian) function with mean RT=500 ms and SDRT=100 ms, (B) an exponential function with $\mathrm{Tau}=250 \mathrm{~ms}$.

Taken from Lacouture \& Cousineau (2008) Tutorials in Quantitative Methods for Psychology 4(1), p. 39. Used by permission of the authors. 
Figure 5. Interaction of SDRT and RSA-Change Predicting Mean Inattentive Symptom Rating.

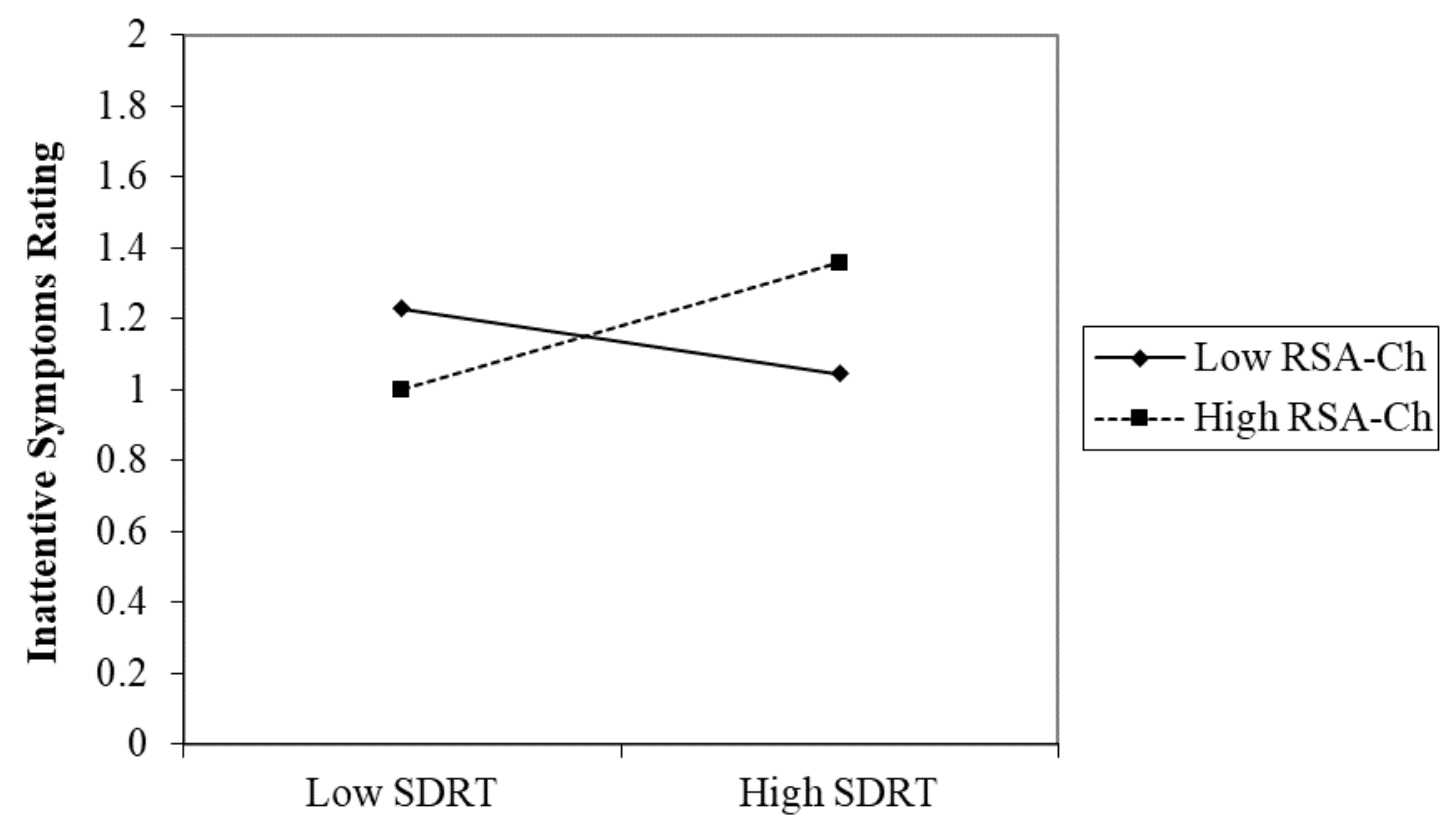

Change in $R^{2}$ due to interaction $=.02$

Note. Inattentive symptoms are from the parent-report DBD-RS and are rated on a 0-3 scale. Low/High SDRT and RSA-Change represent $-/+1$ SD. 
Figure 6. Interaction of Tau and RSA-Change Predicting Mean Inattentive Symptom Rating.

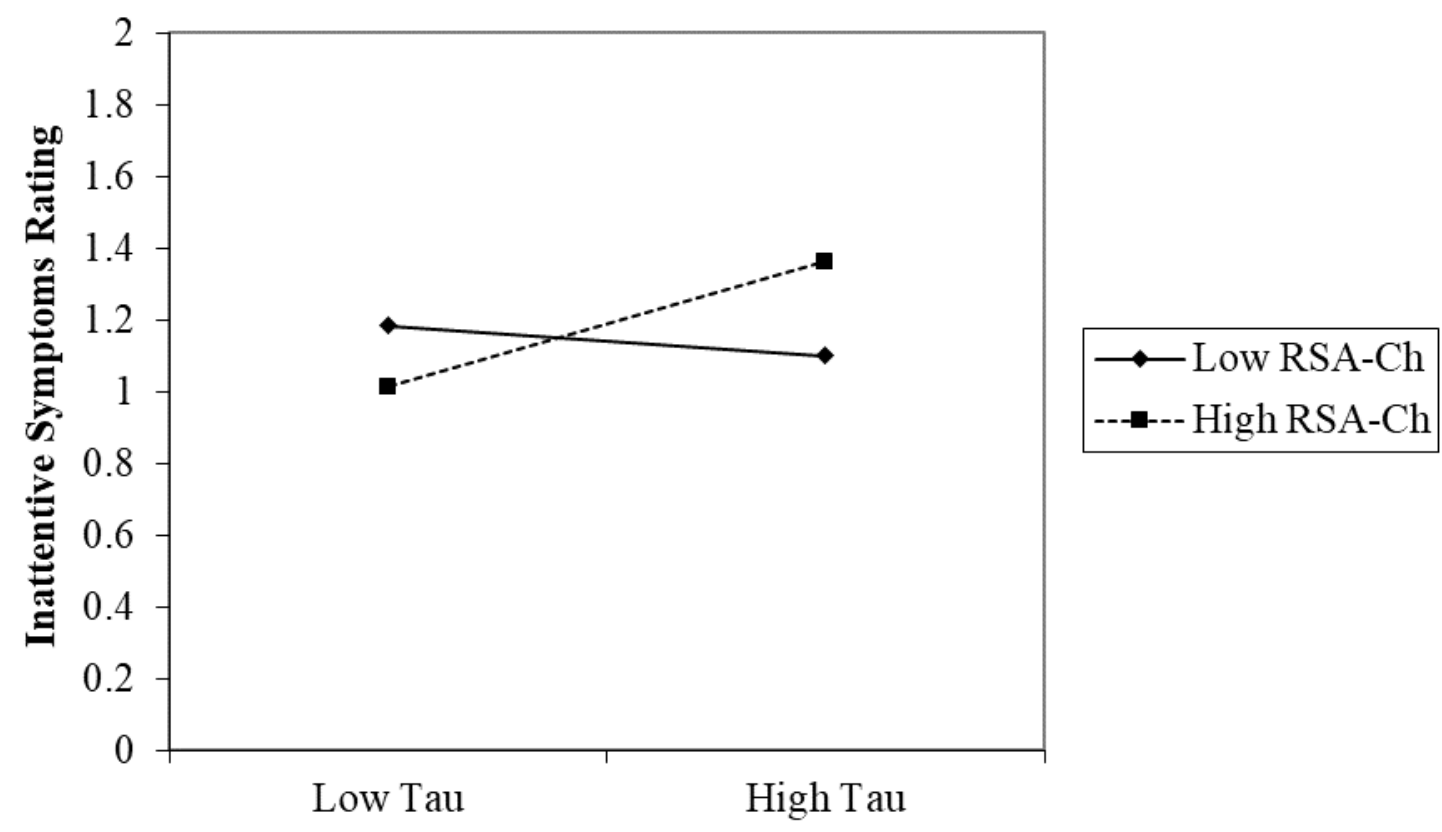

Change in $R^{2}$ due to interaction $=.01$

Note. Inattentive symptoms are from the parent-report DBD-RS and are rated on a 0-3 scale. Low/High Tau and RSA-Change represent $-/+1$ SD. 
VITA

\section{ANTHONY ROBERT WARD}

EDUCATION

2016 to 2019

2015

2011
Doctoral Candidate in Psychology, Florida International University, Miami, Florida

M.S. in Psychology, Florida International University, Miami, Florida

B.S. in Psychology

Portland State University, Portland, Oregon

\section{PUBLICATIONS AND PRESENTATIONS}

Karalunas, S.L., Musser, E.D., Ward, A.R., Nigg, J.T. (2015). Cognitive response style in ADHD as marker of physiological and clinical heterogeneity. Symposium paper presented at International Society for Research in Child and Adolescent Psychopathology, Portland, OR.

Musser, E.D., Ward, A.R. (2015). Stimulant effects on the intersection of behavioral inhibition and emotion regulation in attention-deficit/hyperactivity disorder. Poster presented at the Society for Research on Child Development, Philadelphia, PA.

Musser, E. D., Lugo, Y., Ward, A. R., Tenenbaum, R. B., Morris, S., Brijmohan, N., \& Martinez, J. (2018). Parent Emotion Expression and Autonomic-Linked Emotion Dysregulation in Childhood ADHD. Journal of Psychopathology and Behavioral Assessment, 1-13.

Musser, E.D., Ward, A.R., Tenenbaum, R.T., Morris, S., Feeny, K., Pintos-Lobo, R., Parada-Perdomo, Y., Martinez, J., \& Ester, E. (2019). The roles of salience and value in inattention among youth with and without ADHD. Poster presented at the Biennial Meeting of the International Society for Research on Child and Adolescent Psychopathology, Los Angeles, CA.

Perez, E., Gil, R., Ward, A.R., Tenenbaum, R., Musser, E.D. (2017). Psychophysiological effect of methylphenidate on the autonomic branches in ADHD-diagnosed children. Association for Psychological Science, Boston, MA.

Perez, M., Dominguez, R., Ward, A.R., Tenenbaum, R., Musser, E.D. (2017). Cognitive reappraisal and anxiety predict frequency of cannabis abuse: Preliminary mediation findings. Association for Psychological Science, Boston, MA. 
Sibley, M. H., Graziano, P. A., Kuriyan, A. B., Coxe, S., Pelham, W. E., Rodriguez, L. ... $\&$ Ward, A. (2016). Parent-teen behavior therapy+ motivational interviewing for adolescents with ADHD. Journal of Consulting and Clinical Psychology, 84(8), 699-712.

Tenenbaum, R. B., Musser, E. D., Morris, S., Ward, A. R., Raiker, J. S., Coles, E. K., Pelham, W. E. (2018) Response inhibition, response execution, and emotion regulation among children with attention-deficit/hyperactivity disorder. Journal of Abnormal Child Psychology, 1-15.

Ward, A.R., Bubnick, M.G., Campez, M., Meinzer, M.C., Sibley, M.H. (2015). Sluggish cognitive tempo moderates heterogeneous associations in ADHD comorbidity. Poster presented at International Society for Research in Child and Adolescent Psychopathology, Portland, OR.

Ward, A.R., Alarcon, G., Nigg, J.T., Musser, E.D. (2015). Variation in parasympathetic dysregulation moderates short-term memory problems in childhood attentiondeficit/hyperactivity disorder. Journal of Abnormal Child Psychology, 43, 1573-1583.

Ward, A.R., Alarcon, G., Nigg, J.T., Musser, E.D. (2015). Parasympathetic nervous system dysregulation underlying working memory deficits in children with ADHD. Poster presented at the Society for Research on Child Development, Philadelphia, PA.

Ward, A.R., Musser, E.D. (2015). The effects of extended-release methylphenidate on working memory in children with attention-deficit/hyperactivity disorder. Poster presented at the Society for Research on Child Development, Philadelphia, PA.

Ward, A. R., Sibley, M. H., Musser, E. D., Campez, M., Bubnik, M. G., Meinzer, M. C., \& Yeguez, C. E. (2017). Relational impairments, sluggish cognitive tempo, and high inattention characterize adolescents with ADHD and depressive symptoms. In C. E. Yeguez \& J. W. Pettit (Chairs), Depression and self-injurious thoughts and behaviors in young people with ADHD: Identifying risk processes and informing prevention efforts. Symposium conducted at the $51^{\text {st }}$ annual convention of the Association for Behavioral and Cognitive Therapies, San Diego, California.

Ward, A. R., Sibley, M. H., Musser, E. D., Campez, M. C., Bubnick-Harrison, M. G., Meinzer, M. C., \& Yeguez, C. E. (In Press). Relational Impairments, Sluggish Cognitive Tempo, and Severe Inattention are Associated with Elevated Self-Rated Depressive Symptoms in Adolescents with ADHD. ADHD: Attention Deficit and Hyperactivity Disorders, 1-10. 